U.S. DEPARTMENT OF COMMERCE

National Institute of Standards and Technology

\title{
NISTIR 5107
}

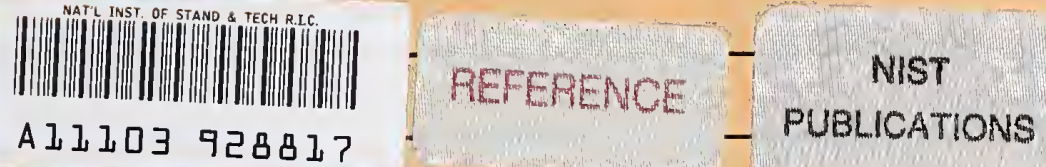

\section{National PDES Testbed}

\section{Report Series}

\section{Proceedings of the AP Validation Workshop}
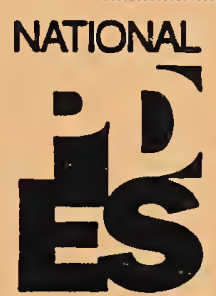

TESTBED TM:

QC

100

.056 



\section{National PDES Testbed ReportSeries}

Sponsored by:

U.S. Department of Defense

CALS Evaluation and

Integration Office

The Pentagon

Washington, DC 20301-8000

U.S. Department of Commerce

Ronald H. Brown,

Secretary

National Institute of

Standards and Technology

John W. Lyons, Director
Proceedings of the AP Validation Workshop
Mary Mitchell

Julie Parker 

Proceedings of the

\section{AP Validation}

Workshop

April 13-14, 1992

organized by:

WG4/P1 Qualification \& Validation Mary Mitchell, chair

WG4/P5 Guidelines \& Framework Mark Palmer, chair

sponsored by:

NIST National PDES Testbed

Validation Testing Systems project

Sandy Ressler, manager

Workshop Proceedings Mary Mitchell, Julie Parker, Eds.

Ellen Trager, Transcription 


\section{Table of Contents}

\begin{tabular}{ll}
\hline Executive Summary & 1 \\
\hline Abstract & 3 \\
\hline Introduction & 5 \\
\hline Call for Participation & 7 \\
\hline Presenters & 9 \\
\hline Presentations & 11 \\
\hline Summary and Workshop Recommendations & 37 \\
\hline References & 43 \\
\hline Attendees & 45 \\
\hline Annex & 47 \\
\hline
\end{tabular}




\section{Executive Summary}

IN THE STEP COMMUNITY the development of Application Protocols (APs) has emerged as a key technical methodology. The development of an AP is a long and complex process. APs are the implementable portion of STEP. This being true, it is vital that implementations of APs are correct.

In addition to an AP implementation conforming to the AP specification, one would hope that the AP is also useful. Requirements traceability addresses the issue of the usefulness of STEP. Do the APs meet the intended requirements? Furthermore, does the AP meet those requirements in a way that can be tested?

This workshop has proven to be most valuable simply by getting the AP developers and testing community together for an extended period of time, discussing these issues. The recommendations produced by the participants and summarized in this document are put forward to the STEP community primarily in the effort to ensure the usefulness of STEP. 


\section{Abstract}

DURING THE COURSE OF THE ISO/IPO MEETING held in Seattle, Washington, in April of 1992, a one-day workshop was held on AP validation requirements and procedures. Included in this workshop were representatives from all approved AP projects and planning projects. The goal of this workshop was to reach consensus on AP validation and conformance testing requirements. The objectives were to establish qualification criteria for AP validation reports; to discuss lessons leamed from initial AP validation activities (i.e. AP requirements validation, ARM validation, and AIM validation); to discuss the proposed improvements to the AP Development and Approval Guidelines; to examine completeness requirements for AP conformance testing; and to discuss the relationships between the components of the AP documents.

This document is an informal record of the proceedings, including the call for participation; the objectives, expectations, and agenda for the workshop; transcripts of presentations; a workshop summary and resulting recommendations, and a list of attendees. Presentations included:

\section{Guidelines on Writing Standards within STEP}

Nigel Shaw, British Aerospace

Status of AP Methods and Documentation

Mark Palmer, NIST

Model Quality Criteria and Metrics Status

Roger Stumps, Boeing

Deploying the Voice of the Customer

Kurudi Muralidhar, ITI - Ann Arbor

What Information is Required in APs to Ensure Compatible Information Exchange?

Jon Aas, FEGS Ltd

Common Methods for PDES, Inc.

Steve Ryan, Core Mechanical Project, PDES, Inc.

Developing and Validating Marine Industry Application Protocols

(on behalf of NIDDESC) Kent Reed, NIST

The Roles of Mapping Tables and Conformance Test Purposes in STEP Application Protocols

Julian Fowler, CADDETC (presented by Jon Owen, CADDETC) 


\section{Introduction}

DURING THE COURSE OF THE JOINT INTERNATIONAL ORGANIZATION FOR STANDARDIZATION (ISO), IGES/PDES ORGANIZATION MEETING held in Seattle, Washington, in April of 1992, a one-day workshop was held on AP validation requirements and procedures. Included in this workshop were representatives from all approved AP projects and planning projects. The goal of this workshop was to reach consensus on AP validation and conformance testing requirements. The objectives were to establish qualification criteria for AP validation reports; to discuss lessons learned from initial AP validation activities (i.e. AP requirements validation, ARM validation, and AIM validation); to discuss the proposed improvements to the AP Development and Approval Guidelines; to examine completeness requirements for AP conformance testing; and to discuss the relationships between the components of the AP documents.

Funding for the preparation on the workshop was provided by the Department of Defense's Computer-Aided Acquisition and Logistic Support (CALS) Office. The work described in this document is funded by the United States Government and is not subject to copyright. 


\section{Call for Participation}

To: AP Project Leaders, WG4/P1 members, WG4/P5 members, and WG6 members

From: Mary Mitchell, WG4/P1 chair and Mark Palmer, WG4/P5 chair

Joint Qualification \& Validation/ AP Guidelines and Framework AP Validation Workshop Announcement

In Oslo, significant interest was expressed in holding a joint technical workshop on AP validation requirements and procedures. The goal of this workshop is to reach consensus on validation and conformance requirements for APs. The objectives of this meeting are:

- establish qualification criteria for AP validation reports;

- discuss lessons learned from initial AP validation

activities (i.e. AP requirements validation, ARM validation, and AIM validation);

- discuss the proposed improvements to the AP

Development and Approval Guidelines;

- examine completeness requirements for AP conformance testing; and

- discuss the relationships between the components

of the AP documents.

There is a need to examine the relationships and traceability between AP requirements, ARMs, AIMs, ARM to AIM mappings and Conformance Requirements. There is also a need to discuss conformance test purposes and abstract test suites.

\section{Call For Position Papers and}

\section{Presentations}

Short position papers ( 3 to 5 pages) are solicited. In addition presentations for the workshop (not to exceed 15 minutes) are requested. Please notify Mary Mitchell by April 1, 1992 if you intend to present material at the workshop. Please email, FAX, or mail papers or presentations to Mary Mitchell by April 6, 1992. For all position papers and presentations which arrive by this date, copies will be provided to all participants at the workshop. Any presentations and papers which arrive after that time will only be distributed in the workshop proceedings.

The workshop will be held at the ISO/IPO meeting in Seattle on Monday afternoon and Tuesday morning, April 13-14. All approved AP projects and planning projects are requested to provide a representative. The convenors of both WG4 and WG6 have approved this workshop. If a representative from your Application Protocol project cannot attend, please notify Mary Mitchell by April 10.

We would like to keep the attendance limited to 25 or 30 people to keep the discussion focused. For this reason, all approved AP projects are asked to send not more than two individuals and all AP planning projects are asked to send only one individual. 
Informal proceedings will be prepared from the workshop. Copies of the list of attendees, all presentations, position papers and workshop results will be distributed to the attendees within a month of the workshop. The proceedings will be available from the NIST IGES/PDES/STEP office, Building 220 Room A127, Gaithersburg, Maryland 20899, attention: Melissa Andrews. In addition, the results of the workshop will be forwarded to Jerry Weiss, the SC4/PMAG chair, for the Seattle Meeting Minutes.

A preliminary agenda is included in this mailing. Feel free to submit comments on the agenda. Thank you for your attention to this matter.

\section{Objectives}

To inform AP project leaders of the status of AP Guidelines and STEP quality methods.

To promote agreement on the minimum requirements for AP quality.

To identify techniques to evaluate AP quality.

\section{Expectations}

Provide a common frame of reference for all AP projects on quality methods.

Produce proceedings with the status of AP Qualification methods and a roadmap for future directions.

Recommendations from each working group:

- Traceability of AP requirements

- AP documentation - improvements and

minimum requirements

- AP project planning and management

\section{Agenda}

Day 1, Monday afternoon

- Introduction of attendees

- Statement of workshop goals and objectives (15 minutes)

- Status of AP Guidelines and AP Qualification Manual (30 minutes)

- Invited Presentations (90 minutes)

Day 2, Tuesday morning

- Working Sessions (2 hours)

Traceability: How to trace requirements to AP implementation?

Documentation: What are the minimum requirements?

- AP project planning and management

Formulation of conclusions (1 hour) 


\section{Presenters}

A total of eight people made presentations.

Guidelines on Writing Standards within STEP

Nigel Shaw British Aerospace

Status of AP Methods and Documentation

Mark Palmer NIST

Model Quality Criteria and Metrics Status

Roger Stumps Boeing

Deploying the Voice of the Customer

Kurudi Muralidhar Murali ITI - Ann Arbor

What Information is Required in APs

to Ensure Compatible Information Exchange?

Jon Aas FEGS Ltd

Common Methods for PDES, Inc.

Steve Ryan Core Mechanical Project, PDES, Inc.

Developing and Validating Marine Industry Application Protocols

Kent Reed NIST (on behalf of NIDDESC)

The Roles of Mapping Tables and Conformance Test Purposes in STEP Application Protocols

Julian Fowler CADDETC

presented by Jon Owen CADDETC 


\section{Presentations}




\section{GUIDELINES ON WRITING STANDARDS WITHIN STEP}

\section{Nigel Shaw British Aerospace}

\section{Editing Committee Chair}

My starting point is the review meeting held to develop a UK consensus on the Part 203 ballot comments. A number of comments described the fact that it is a difficult document to comprehend. It became clear that for all APs, STEP developers had quite a way to go in turning these documents into usable standards. The concern, apart from reader difficulty, was that the mapping between the application requirements in the ARM and how they are represented in the AIM. This mapping is extremely difficult to present. There are a lot of complex ideas represented with little explanation. There is also a concern over the shear length of these documents. There was little information in an AP that led you through what an AP is and how the various components of the document relate to each other.

Depending on your perspective, e.g., I am an implementor or I am a user or I am a testing person, to some extent biases the way you want to go through the document and how you interpret the relationship between the different sections. Further, there were editorial problems like the definitions were not included from the integrated resource (IR) Parts but the entities were. In other cases, examples and notes from the $\mathbb{R}$ documents which were copied into the AP turned out to be inappropriate to the AP and so forth. So it was very clear that we needed to tighten up considerably the guidelines provided for APs. In Oslo our first attempt was made to accomplish this. What it really did was generate issues for debate at this meeting. I'll try to touch on some of the things that we were covered at the AP Guidelines meeting on Saturday. However, the improvements to the Supplemental Directives and AP Guidelines documents are far from final. Mark Palmer mentioned the concern about an AP does something for the user, it has a purpose. Implicit in that is the user shall do certain things to comply with the standard. At the moment, APs aren't really spelling that out very clearly. They say - "you must have a conforming system", "this is what you must do". What these documents need to do is describe the process of using this AP. The AP document needs to provide the answers.

Its been pointed out that for the first release of STEP, there are a very limited number of things that an implementor needs to do to conform to the ISO 10303 standards. Those participating in the $A P$ Guidelines meeting felt it was important to state in the introduction what a particular AP is intended to do. Furthermore, it should state the industrial needs that led to the Aps development.

An AP also needs to explain what an AP is. The reader needs a road map to lead them through the interrelationship between the components of the AP. It is very important to clarify the scope with respect to what kind of product an AP document and the reader is dealing with. The intent of the Editing 
Committee is to include boiler plate text for the introduction and almost all components of an AP to make it clearer how the components relate, where the requirements are specified within the document and on whom the requirements are placed.

The Editing Committee is looking to improve and include boiler plate text and other pieces of information required by Part Editors in the Supplementary Directives. This work will be coordinated with the Guidelines for AP Development for all areas of the Application Protocol. The inclusion of Application Interpreted Constructs (AIC) in the AP document is a major open issue, especially from the documentation viewpoint, that needs to be resolved.

STEP needs to ensure that the context is very clearly stated. The type of products the AP is dealing should be is clearly established. The implementation matters such as who shall do what and how. There is a missing detail which needs to be addressed in the AP specifications. They should state when do the requirements get imposed.

As a very large group, the participants of this workshop are trying to improve the quality and quantity of standard text as required in application protocol documents. As Editing Chair, I want to get STEP documents consistent. I am a firm believer in the idea that if someone has read one AP that fact is going to help them understand the next one. The Editing Committee will to be providing in the Supplementary Directives, hopefully by the end of May, a large amount of the required text for Application Protocols.
Question and answer period.

\section{Main Points}

Focus is on how to make AP documents into usable standards.

Mapping from application requirements in the ARM and representing them in the AIM is extremely difficult.

Other concerns include the sheer length, the lack of description of how the various AP components are related, and editorial inconsistencies across and within documents.

Roadmap needed to lead reader through the components of the AP and through using the document. 


\section{MODEL QUALITY CRITERIA AND METRICS STATUS}

\section{Roger Stumps Boeing}

member of the IPO Dictionary Methodology Committee

Last week the IPO Dictionary Methodology

Committee held a three day working session at

Boeing on the Model Quality Criteria document to make sure that the ideas in it are still valid with the current STEP development process.

The first item for discussion is the current state of the Model Quality Criteria document, and the changes that are proposed to be made to it. Next I will discuss the future enhancements to accommodate refinements in the STEP development process. Finally, we need to develop correlations between the multiple quality metrics and to extend the process maturity and address work going on in the STEP implementation areas. The document name will be changed to Multi-Quality Criteria Metrics to add the idea of both the identification of quality criteria and a way to measure this criteria. The objective is to provide a meaningful measure of what a STEP model status is.

There is an issue of what categories of quality documents are needed verses what type of document we currently have. The viewpoint of the committee is that a series of documents are needed. It was proposed that the Model Quality Criteria document be worked on to identify what needs to be measured and what additional metrics are needed for them. In addition, a quality procedure document is needed in order to apply the criteria. A practices document, like the AP Qualification Manual, is needed with forms to be filled out when evaluating a STEP model. Each document should have a section which specifically focuses on a certain type of model. Those are the three categories of documents that we are looking at right now.

Our committee proposes to continue working on the document framework the quality management methods in STEP with the available resources. We plan to pull together an outline of what we think the overall document architecture for quality management in STEP should contain. It is a place to start and throw darts at.

Concerning the practices and procedures categories, there has not been too much energy on the committee to actually work on that type of document at this time. (These categories are being developed by WG4/P1.)

We need more feedback back on our document. We have been getting a lot of feedback but not always at the optimal time for us due to time constraints and other constraints on people. The practices documents developed by WG4/P1 and P5 have been coming back into the committee but we also need joint discussion between the projects on the new thoughts coming in these documents and better coordination. We see our committee as belonging at 
this level - producing the requirements and assisting in their deployment by WG4/P1. There are also the practices committees that are assessing the documents and deploying the results. We do not have any overlap in effort however, the two committees should be working together. We think this is missing because of time constraints. Once again, feedback is very important to keep these documents alive and working as STEP defines and refines its methods.

We have received requests for our project to develop examples of what the elements of the STEP documents should look like to help the model owners build in quality throughout the process instead of trying to improve the documents at the end of the process. We are thinking of adding a section to the document on defects. We will be putting together some guidelines and examples about how to spot and correct some of these type of defects and how to prevent them from occurring in the modeling.

Within that concept, we have found that there is a problem with qualification criteria QC 8, model syntax evaluation, and QC 9, formal semantic representation. We are working on a framework for them to see how they work together. The sections on QC 8 and QC 9 will be totally rewritten in the next version of the Model Quality Criteria document.

The committee's strategy is to identify and categorize common modeling defects and to build template solutions to common modeling problems. WG4/P2 used this approach for some common integration problems. These will be documented in modeling practices guidelines, such as the Express usage guidelines. We are hoping that when the model owners develop their models and run into problems, they go through the defect listing. If they do not find the defect and a solution that matches their construct, they should be encouraged to come back to the dictionary and qualification practices committees. They should describe what they are trying to model and propose their solution good or bad. They should be provided with help to determine if there is a better way of modeling this. The communication with the model owners needs to come back to our group too and not just to qualifications practices. We need communication between practices and model owners back into the requirements for the metrics and criteria.

The time available for work on this document is during weekly conference calls that will start next month. If anyone is interested in participating in those, let me or anyone else on the committee know. We will be glad to keep you informed of when the conference calls are. We are also planning to have some interim releases between now and the next document release. The version 1.3 Model Quality Criteria document will probably be available in October.

Question and answer period.

\section{Main Points:}


Keep the "Model Quality Criteria" current with STEP methods and the development process.

Creating a document framework for quality management methods in STEP.

A series of documents are needed covering quality criteria and metrics, procedures, and use of quality management methods in STEP.

Dictionary committee needs more feedback and coordination from model developers and qualification practices.

Qualification criteria for model syntax and use for semantic representation are being enhanced.

Model Criteria document will incorporate section on common modeling defects and recommended solutions.

Document will be re-titled "Multi-Quality Criteria Metrics" and published in October 1992. 


\section{Status of AP Methods and Documentation}

\section{Mark Palmer NIST}

WG4, P5 AP Guidelines and Framework Project Leader

\section{Overview of AP Progress}

To establish the context for the discussions on AP validation, I want to go over the activity diagrams for the AP Development Process, the responsibilities of an AP project and the component elements of an AP. With this information we can identify where AP validation plays a role in the process. From a high level, we have three primary steps in developing an AP. First, plan and develop the AP. Next, develop its abstract test suite. Finally, the development of a prototype AP implementation is strongly encouraged prior to the release of the AP for DIS ballot. Decomposing the plan and develop (A41) activity, we have five basic steps: develop scope and requirements, develop the Application Reference Model, develop the Application Interpreted Model, develop conformance requirements and test purposes, and then complete your AP document.

I will review the current state of the documents which focus on Application Protocols. The three documents currently being worked on within $\mathrm{SC} 4$ are: Guidelines for the Development and Approval of STEP APs, WG4/N34; Issues and Recommendations for a STEP AP Framework, a NIST document; and the STEP AP Qualification Manual, working draft .05. The AP Qualification Manual and the AP Guidelines for the Development of APs have been moving forward in parallel. There have been modifications to both to keep them synchronized. There is work being done on the next AP Framework document. And as a result of meetings of the Qualification Project, WG4/P1, there is a recognition for the need of a Quality Management Structure. The Qualification Project is trying to evolve from a state of inspection at the end of the process to providing the necessary tools for quality management as the AP components are developed incrementally. The AP Guidelines describes three groups of components in an AP Group 1, with the scope, requirements, ARM, and ARM validation report. These components are qualified prior to proceeding into the development of an Application Interpreted Model (AIM). The AIM is developed and also validated and the elements are qualified as Group 2 before developing conformance requirements and test purposes. The conformance requirements, test purposes and the completed AP documentation is then qualified as Group 3 prior to submission for review and approval by the SC4 Editing Committee.

The requirement for AP validation has always been recognized but not fully exercised in existing AP projects. AP validation is an identified task in the development of an AP and plays a critical role in delivering usable APs to the STEP community.

When I come to ISO and IPO meetings, I try to have a current list of AP development issues. I have extracted from that list the issues that are applicable 
to AP validation so you have some issues with which to start these discussions. I will briefly go through these issues. If anyone wishes to question the merit of these issues, please raise your concerns at this time.

There is the issue of verification of the international consensus on the AP scope and requirements. We have a process and procedures defined for that task but I do think it has not been successfully exercised in all arenas. There are cases of overlapping APs and conflicts in what are the industrial requirements that are the driver for any AP. Under the issue of ARM development and validation, there are issues on the required specificity of the application semantics and the required level of detail for the ARM.

Likewise, there is still work being done on the rules for defining Units of Functionality. How does one verify the validity of those Units of Functionality within the domain of the AP? There is also an issue on the role of these "functional building blocks" in an AP. Some of the APs have functional levels in their APs, i.e. how do we build upon a core ARM to provide increasing levels of functionality. This has ramifications on all the downstream activities that must support the AP. On AIM development and validation, there is a fundamental issue of ensuring traceability from the requirements to the AIM and eventually to the ATS. We do not have the tools and the methods in place to ensure comprehensive traceability. Likewise, we have the issue of how to prepare the EXPRESS expanded form from the short form. There are some verification issues here as well. Likewise, there are issues on how to assess the AIM and the need to define procedures for building and validating AICs.

Moving on to test purposes, abstract test suites, and executable test cases, we have a similar set of issues on granularity as with the ARM. There are also issues with the viability of one set of test purposes being useful for multiple implementation forms. Finally, under the issue of what I classify as AP documentation, there is the issue of expanding the mapping table. The initial role of the mapping table was just to identify each ARM concept and how the application concept would be represented by the AIM construct. However the constraints on how this AIM construct is used are equally important.

One of the insights that came out of the Oslo meeting was that if we actually expanded that mapping table to a sufficient level of detail, the mapping table could provide much of what is now required in the test purposes, i.e. one would identify each unique path and that would be the testable structure for clause 6 in the APs. The issue is making the mapping table more robust and more understandable so that some of what is in the mapping table does not need to be repeated in another section of the document. The issue on enforcing validation and the documentation of the AP validation has been raised already.

There are also issues on the ability to obtain all ARM concepts from the AIM constructs, i.e., to promote completeness by generating the reverse mappings. There is also the issue of the contents from the AP usage guide. Much of the work today has been looking at what is a compliant 
implementation of an AP. There is a corresponding issue of how does one ensure compliant data sets? It is in the delivery of compliant data sets that the AP usage guide may finally play a critical role but we have not clarified that issue. This has been a brief description of the issues which confront the development of validated APs.

Question and answer period.

\section{Main Points:}

Endorses the development of a Quality Management Structure for STEP.

The requirement for AP Validation has not been exercised by all existing AP projects and this raises quality issues.

There are many unresolved issues with respect to AP development \& validation.

Qualification is evolving from inspections at the end of the process to incremental evaluations and more direct assistance to Part Editors.

Proposal to have an ISO ballot to determine if there is international consensus of the AP scope and requirements. 


\title{
Deploying the Voice of the Customer
}

\section{Kurudi Muralidhar Murali ITI -Ann Arbor}

\author{
Member of WG6, Conformance Testing
}

\section{Tracking Requirements for Applications}

One of the methods that has been used in tracking requirements in industry is called Quality Function Deployment. This method works fine for tracking the requirements of all types. In addition, it provides for the prioritization of requirements in a structured approach. This technique is similar to other structured requirements approaches. QFD may not be the best technique but it allows you to track and analyze the requirements, $\mathrm{QFD}$ provides a problem solving group with a system for developing a common understanding. The best thing we found out about using this method was the common metrics it provides. The teams which have used it are comfortable that they are working with the same meaning. That is where it helped.

There are various business needs being addressed by STEP. There are maybe similar business needs between particular APs. What we are trying to achieve could be a functional requirement; functionality could be a business need. There may be several reasons for achieving this particular requirement so we can track them with $\mathrm{QFD}$ and we can assign an importance number for each of these needs. The first step in the process is identifying the requirements, defining them, and then collectively assigning a priority what is important about them.

How important is using a formal requirements definition and tracking method? A method like this helps to build a consensus among groups of people and can help you to finalize what are the best requirements. What are the highest needs? Based on evaluations of the various approaches for a particular solution or how you want to meet the particular requirement, you can start assigning rules so you can get absolute measure of importance.

Later in the process when you have actually finished your AP, you can take a particular requirement from applying this method and you can track it all the way through and see how far the requirement will really implement.

To provide more STEP type of things from a general product design, we used a QFD approach for the analysis of an abstract test notation for the abstract test suites. Abstract test notation is a language use to describe abstract test cases for APs. In applying this approach, there were several languages that were available for use as an abstract test notation. We looked at the various requirements for such an abstract test notation. It should have some type of ability to manipulate the instances of product data so that they can test the validation of an AP. There is some testing related administrative information like where this test came from. QFD was used to establish the high level requirements for the notation for specifying abstract test cases. This is important 
so that we can prove the capabilities that a language has matched the requirements and that the language has something we want. We also assigned importance to each particular requirement in a collective fashion. QFD helped us direct our focus to the features that a candidate language most needed to support.

Question and answer period.

\section{Main Points:}

STEP should consider using a formal requirements method to ensure traceability.

QFD provides the ability to trace a requirement from its inception through the deployment of a solution.

QFD was used for the capability survey of potential abstract test notations.

The metrics within QFD provided a means of measuring the relative advantages of each of the abstract test notations. 


\section{WHAT INFORMATION IS REQUIRED IN APS TO ENSURE COMPATIBLE INFORMATION EXCHANGE?}

\section{John Aas FEGS, Ltd.}

Member of AP 206 Wireframe Representation Models

I would like to address what information is required in APs to insure compatible information exchange.

Experience has told us that definitions which are too loose provide too much freedom to build what implementors like or what implementors can understand from the document. IGES has a bad reputation because this freedom created limitations that were not very good-the information exchanges between systems were incompatible. A lot of effort is going into STEP to remedy these weaknesses-to fill the openings and to make it less likely that people will interpret into the documents what they would like to do. What I would like to present today is the fact that despite this effort, there are still some openings for interpretation that need to be filled. I will point out a few that the AP 206 team has found by experimenting and doing actual data exchanges. Due to limited time, I will address one aspect within each of these issues:

1. conversion

2. entity mapping

3. application model characteristics preservation.

To begin with, STEP does not have a clear picture of how the implementation of STEP technology should be done. Our team thinks that implementations will be done which produce systems of a special kind which have a well defined boundary but relatively small scope, e.g. a manifold solid modular. Users will create product definitions and extract product definitions from a system of the same specialized type-essentially, systems that have equivalent functional capabilities. The data exchanges in general will not be across functional capabilities. What does equal mean? Due to practical limitations, a user will actually be sending models between different applications because the implementation may internally represent information differently than the STEP representation. Secondly, a systems implementor wants his system to be unique. The system's distinguishing features are what sell the implementation. In STEP, we are talking about the structure for representing product information where we are exchanging models between different kinds of systems internally even though they have the same STEP interface. APs have to find a way of describing a conversion from one type of system to another. APs need to address the conversion aspect and not just leave the interpretation of what an acceptable conversion is to individual users and implementors of the AP documents.

The next issue is the mapping of application requirements to the STEP integrated resources. First 
an example from Part 205, there are three types of surfaces geometric representation models supported in Part 205. The three representation models are 3-D surfaces model, a face-based surface model, and shell- based surface model. Typically in a direct, simple transfer, a surface modeler has the full topology. With instructions, we can make this topology into a bounded surface model and it can be converted to a manifold model- i.e. a model with no more than two surfaces meeting at each edge. If you have surfaces that meet at more than two faces at the same edge you can map that to a non-manifold surface. There is the freedom to map a geometric representation model of one category into several alternative representations.

\section{Recommendations:}

- Limitations of the way STEP integrated resources are used are necessary.

- Where possible, only a single mapping should be used.

- Any options should be fully described including any implications on conversions.

- The existence of undesirable flexibility is very valuable feedback to the definition of the AP and this should go into an AP. The contents of APs should include enough to plug all holes.

- We should perform manual testing on the AP.

- We need to make sure that all available knowledge will be reflected in the AP documents.

- We must collect experience with AP guidelines.

- We should collect and document all of our bad experiences and share them with STEP developers and users.

Question and answer period.

\section{Main Points:}

Implementation systems may continue to use internal representations of product models that differ from STEP.

An AP should describe the requirements on any conversion of one entity representation to another and not leave this open to the interpretation of implementors of the AP.

Actual testing of data exchanges of the representations prescribed by the AP should be done prior to AP standardization.

Need to accept that implementations will convert between their internal representations and a STEP representation and define the constraints needed to ensure meaningful data exchange. 


\section{COMmon Methods For PDES, INC.}

\section{Steve Ryan}

Core Mechanical Project - PDES, Inc.

Mary asked me to talk a little about common methods meetings that were held within PDES, Inc. during the last 3-4 months starting in December of 1991. The objective of this project was to bring a half dozen project leaders together to agree upon the techniques that each of the projects would use in the future. We looked at the different approaches that we were using to develop and test CDIMs (Context Driven Integrated Models). Within PDES, Inc. we have been doing this to help us provide feedback into the STEP community on the problems with resource models. However we also consider the CDIMs precursors to APs. The idea of this project was to look at the different approaches we have been using with the five projects going on in PDES, Inc. that are developing APs or will be developing APs. We want to make them look more cohesive and comprehensive, five separate application projects. Each had separate ways of doing the job.

A comparison was made between the CDIM and the AP processes. We found considerable similarity at several steps in these processes even though the overall objectives of CDIM \& AP processes were very different. We determined that the scope and requirements activities did not need to differ.

[No further transcript is available: the tape was overwritten inadvertently.] 


\section{DEVELOPING AND VALIDATING Marine Industry Application Protocols}

\section{Kent Reed NIST (on behalf of NIDDESC)}

Member of Navy Industries Digital Data Exchange Standards Committee

I am in the Building and Fire Research Laboratory at NIST (National Institute of Standards and Technology) and I am here to report on work done by the Navy Industry Digital Data Exchange Standards Committee (NIDDESC) which is a cooperative effort of Marine Yards and the U.S. Navy's Sea System Command. This is work I participate in as a technical advisor, most of the "hard" work is done by others. These individuals include: Doug Martin from NASSCO, the model owner for a number of proposed STEP application protocols; Mike Gerardi from Bath Iron Works and Mike Polini from Jonathan Industries; Rick Lovedahl from Lovedahl and Associates; and Dan Wooley from Newport News Shipbuilding. A number of the testing items I will discuss are actually being done by Subhash Ramachandran and Bill Schmidt of Angle, Inc.; Jack Brainen and Lisa Deeds of David Taylor Research Center, and others.

The Navy work is broadly scoped. A series of six application protocols were proposed by NIDDESC in Oslo as STEP work projects. The AP proposals cover the topics of ship piping systems; heating, ventilation and air conditioning systems; and ship electrical raceway systems; ship structural systems; ship outfit and fumishing; and ship parts libraries. All share the common heritage in building industry applications.

All of these applications have a design context. All focus primarily on the context of exchange. The Naval Sea Systems Command exchanges design data with its yards and the yards exchange data with other yards as the products are designed. All the application protocols have to deal with configuration management of the systems and parts as they are being designed.

We have closely coupled elements in each one of these application protocols. For example, the scope and requirements relate to the application reference model (ARM), and the application reference model maps into the application interpreted model (AIM). Test purposes, performance requirements, and other AP components are elements that we haven't talked about yet. They are all strongly interrelated but in fact they are developed separately. Some of our problems are due to the fact that multiple versions of application models are under development at any one time. There are parallel activities; one laboratory may be analyzing one version of the model while its developer is working on the next version of the model so both teams may be looking at the next and preceding versions. At the same time, the multiple application protocols have common requirements as in the case of distribution systems for piping systems 
and for heating, ventilation and air conditioning. There is a massive amount of documentation to manage in the existing paper-based model development world. It is a massive problem to maintain the consistency of that documentation. Let us look at some of these elements. Partially overlapping scope and requirements, developed by a part owner, are reviewed and revised by NIDDESC members at many different meetings. NIDDESC validates this work by going to different domain experts to examine the scope and the requirements. If they agree that these requirements are really what needs to be specified for this application, we proceed to the next step in the AP development process. Over time we have had trouble keeping the scopes and requirements consistent with the existing state of the models.

Definitions are handled in much the same way. The part owner has the primary responsibility for either writing or collecting definitions. Definitions are revised in light of model testing and simply reviewing them. Again, the validation consists of having different people look at the definitions and agree on what was meant and that this is what was required. Part owners maintain the definitions in a word processor. The difficulty is that part owners are defining everything and they have other things to do. Typically, there are not sufficient definitions for the entities that exist. Over time the model entities may drift from their definitions. Unfortunately, the entities with missing definitions may sound like entities in other models. Model owners assume that the definition for the series of APs is the same. We are have the same trouble keeping the definitions synchronized, just as we have in keeping the definitions synchronized within the Parts of STEP. We have trouble just accessing the definitions that are current.

Application reference models have consumed the bulk of the development effort. Typically the part owner developed the draft application reference model. These models have been built in NIAM, Nijssen's Information Analysis Methodology. Nijssen has refined his ideas over 10 years. The software tools we use to support NIAM differ from each other. The models have been critiqued at many workshops and are now being revised in light of the testing results. We have tools that analyze NIAM models for consistent and correct syntax. The tools used include PC-IAST, a Control Data Corporation product, and Ridl* from Intellibase Corporation, Belgium. Both of those tools generate SQL schemas which we use for model validation testing. The ARM graphic representations are maintained in the part owner's word processor format as drawings. Their controlling representation is maintained in the repository of the NIAM tools.

One obvious problem is maintaining a consistent system of ARMs across the scope of multiple APs. Since no tool does everything we need, we must maintain multiple representations, one for each tool we use. To further complicate matters, the tools have different naming conventions. Since we are testing the ARM in a relational database environment, we have no direct mechanism to test the constraints. The major problem for us is that it takes a tremendous amount of human interaction to revise a model and then transform these revisions into the formats that each of the tools require. 
To move on to the ARM to AIM mapping table, each one has been developed primarily by the part owner manually, without any software to generate it. The mapping table is then revised in light of the model enhancements and the testing results. The AP Guidelines are not very clear on the requirements for the mapping table and each example we have looked at from the emerging APs is different from the last.

Validation of the AIM has proceeded only to the extent that I have tried to compile some of the Express schema into the Data Probe, a prototype software tool which is available through the Manufacturing Engineering Laboratory and National PDES Testbed. Next, we will populate these models with data from the ship yards. We decided that going back to a relational data base environment at this point is too much effort. The AIM is maintained in a word processor. At some point we will need to include EXPRESS-G drawings, if we can find a tool to build the EXPRESS-G with. I would like to point out that test case data used in AIM testing are the same data that we used for ARM testing.

In the sections on conformance requirements and test purposes, I will ONLY speak on usage test purposes at this point and not the more basic structural test purposes. They have been drafted by part owners and reviewed and revised by other team members. The validation of conformance requirements and test purposes consists of a critique by domain experts. During this process, we start to gather test case data from domain experts along with data from the testing teams who use existing Navy projects as their source for data. For example, the piping system testing information is coming from the design of the combat information centerfor the DDG51. In the test purposes, their definition is a problem. The source of the problem is with the process, the requirements have changed at an alarming rate.

The primary problem for us is lack of a good environment to help us control of our information. We have multiple representations of the same ideas because we operate in different environments. We have tried to use the best tools but these offer only fragmented solutions. We lack tools that support model validation testing. The tools that do exist are not yet industrial strength; I have broken them all. This is a common complaint about the software industry but it hurts the development of STEP where so much complex information has to be captured and manipulated. The tools are not validated to work correctly so there is always the question "Are we testing the tool or the model?". In using the available EXPRESS compilers, a lot of the problems have been worked out. They are still one-way processors. We developed filters to move from one model environment to another model environment but this is a one-way process. If we change something between ARM and the AIM, it is very difficult to propagate that change backwards to go back to earlier steps in the AP development process and revalidate the changes is very time consuming.

In spite of all this, the message I would like to convey here is that we believe in STEP. There really is no other alternative. NIDDESC and others are trying very hard to make STEP work. We must, however, find ways to make this work easier and less human resource intensive. STEP developers need to take advantage of everything that is available but I 
don't think this is sufficient. We will need a lot of automated tools to get the job done. Paper-based management of AP development is not enough and it requires too many scarce human resources. There needs to be a concentrated effort to develop a software environment to support AP development. This would be cost effective and improve the chances of the success of STEP. The current documentation in the AP guidelines and other STEP guidelines are not sufficient to provide adequate direction to STEP developers. This causes each development project to hash through the same set of problems and ambiguity. We should take the time to document the best known practices in these documents now.

Question and answer period.

\section{Main Points:}

NIDDESC is developing six highly integrated APS for the marine industry.

The environment for developing an AP is fragmented which makes it difficult to maintain a consistent set of AP components.

Scope, requirements, and definition are validated by reviews from domain experts.

Both the ARM and AIM are validated by populating the models with real world data.

Software tools support for AP development must be improved.

STEP Guidelines documents must be enhanced to reflect lessons learned and the current best practices for development. 


\section{The Roles of Mapping Tables and Conformance Test PuRposes In STEP APPLICATION PROTOCOLS}

\section{Julian Fowler CADDETC Presented by Jon Owen CADDETC}

Member of AP 201 Explicit Draughting

Convener, WG6, Conformance Testing

I will go through the thoughts that CADDETC has had on the role of mapping tables and conformance test purposes in STEP application protocols. This has been largely inspired by Julian Fowler from his role in Part 201, Explicit Draughting. First I will provide background and ask the question "What is an AP?" This answer to the role of the mapping table and test purposes is not guaranteed but it's a start. We will examine the relationships between the components of an AP. Then we will look at the role of the mapping table; what is in it and what the prospects are for moving forward on an improved representation for it. The last half of the presentation will look at the test purposes; what they are and how they are documented. Then I will try to bring the importance of these components together in the summary.

Mapping tables and test purposes were required components of APs from the start. In practice, the work on APs to date has concentrated on the activity models, the application reference models, and in particular the AIM development. The approaches to the mapping tables and the test purposes were not agreed to or documented. That is evident from the differences in the appearance of Part 201 and Part 203. Julian is not here today but consider all the mapping tables he merged in proposing an improved mapping table format that could support the generation of test purposes and an abstract test suite. I believe the mapping table format can and I believe it has emerged out of the integration processes. How can we ensure that the AIM actually satisfies everything that is in the ARM. I will summarize where we are and give a few suggestions on how to move forward.

What is a STEP Application Protocol? It is a standard and a specification of the data constructs that are required to exchange all the information needed to meet documented industrial requirements.

In practice, an AP may have several levels of conformity. It is important that each clearly identified need should be documented in separate AP level. The levels of functional capability within an AP are kind of a miniature AP within the AP.

The STEP implementation combines an AP and implementation form, such as Part 21 - the Physical 
File, or SDAI.

An AP is not a subset of the integrated resources as has been thought of in the past. What we need to do is to integrate the AP so that we can enable interoperability between different APs.

We have a mapping table that brings together the information requirements in the ARM and the AIM as well as the conformance test purposes. The test purposes exercise the structures and requirements in the mapping table.

A refinement of the previous slide is that we can see an AIC as a complete testable element which can be shared by more than one AP.

The idea behind the mapping table is to document the correspondence between what was in the information requirements in the AIM. The mapping table is really providing a link between an application domain and its language. An observation is that the length and the level of detail in the mapping tables will depend on the details of the AP information needs. If you have a very detailed Application Reference Model then false detail will be presented in the mapping table. Part 201 has a two-level mapping table. The content and layout has not been agreed to but this is one way to produce a mapping table. Each construct of the ARM is matched to a primary construct in the AIM. That works for Part 201 and it may be sufficient for an AP with a mapping that has a direct interpretation of integrated resources. APs such as Part 204, Part 205, and Part 206 have requirements which are very close to the representation provided by Part 42.

The mapping table to date has tended to be a - I've got something here in the ARM that I need to satisfy and here is some stuff either of the one-liner or a collection of things that fulfill it. It might be quite useful in our environment to be able to have the mapping properly directed back to the ARM. If I have an instance of something in my physical file, what is that actually doing? You get a pointer back that says something like in application-speak "I am satisfying this requirement".

We have the AIM in the EXPRESS language. It is useful to make the mapping tables computer processable. The question here is do we actually need a standardized formal method for documenting requirements? Possibly..Probably...

Conformance test purposes are the starting point for denoting an abstract test case. It provides the objective that you are trying to test. If you take the test purpose, a particular bit of the mapping table exercised by the test purpose, and then the information requirements from the AIM that it naturally brings in, that specifies the things that you need in order to be able to test a particular test purpose. Conformance test purposes identify all discrete options that are in the application.

That process for defining conformance test purposes is documented in WG6 document N26 written by Julian Fowler for this workshop. It is feasible to produce the whole set of required test purposes from the AIM automatically. We have rules for mapping EXPRESS to generate the stand-alone discrete 
options to be tested. The other half has yet to be documented. Just because of this problem with the mapping table - we can express test purposes in STEP-speak but not as it relates back to the ARM in application-speak. A word on the test purposes for AP 201. Test purposes were grouped in a particular way. Test groups are structured by units of functionality, then there is a series of test purposes for product associations. This is not AP 201 specific. We anticipate that every AP is going to have these test groups because they are part of Part 41.

Next, how are the test purposes being documented? Test purpose name is under the corresponding AIM entity. Where that entity participates in a role is the context and they are listed. There is a close relationship between the mapping table and the test purposes. CADDETC has an algorithm for generating test purposes from a given EXPRESS specification. It does not include consideration of defects in global and local rules so that if there is an attribute that says optional the program will generate a "present" or "not present" with a local rule that says where it takes it - which means it's always there. But in practice the algorithm generates the maximum set.

The mapping tables include better identification of the AIM to ARM mapping rather than the ARM to AIM. The technique and a given mapping table can fulfill that because we have this half of the automation. If we can get the other half, then we can move the conformance test purposes in the AP into the abstract test cases because there is a formal and sufficient basis for doing that. The technique will always be reproducible.

Finally, a brief word on what has been called usage test purposes. There are tests around which exercise specific combinations and potentially complex combinations of data in the AP. We believe that such complex test purposes should be used before you move into conformance testing. For example, if I am a customer who uses this AP to do the sort of things that I want, then I want a system that does $A, B, \&$ C. You can specify a usage test to make sure that you get the information you need within a particular AP or a particular implementation. You can then say this: Is it what I need? Does the model actually do it correctly? So the recommendation here is that each of these test purposes should be used as part of the AP evaluation.

Question and answer period.

\section{Main Points:}

An AP may have several levels of conformity; each should be documented in separate AP level.

Mapping tables bring together the information requirements in the ARM and the AIM solutions to them as well as the conformance test purposes.

Conformance test purposes are the starting point for specifying abstract test cases.

Mapping tables include better identification of the AIM to ARM mapping rather than the ARM to AIM.

We can move the conformance test purposes in the AP into the abstract test cases because there is a formal and sufficient basis for doing that. 


\section{Summary \& Workshop Recommendations}

\section{Working Sessions}

The participants were divided into three working sessions:

\section{Ensuring Requirements Traceability}

Steve Brett, Mitchell Gilbert , Jochen Haenisch, Mary Mitchell, Kurudi Muralidhar Murali, Kent Reed

\section{AP Validation Requirements and Documentation}

Diane Allen, Allison Bamard, Bill Burkett, Larry McKee, Haidee Rapacke, Bob Street, Anne Williams

\section{AP Project Management and Planning}

Jon Aas, John Barnes, Peter Kruse, Mark Palmer, Sandy Ressler, Bill Russell

\section{Action Items}

The action items which were generated during the workshop fell into four categories:

- improvements to existing documents, such as the

AP Guidelines and AP Qualification Manual

- refinements to the AP development process,

- requirements for additional WG4 documents or actions needing WG4 attention, and

- actions which need attention by groups outside of WG4.

\section{Identified needs and potential sources to assume the responsibility:}

- Quality management structure which covers the AP development process and STEP methods. (WG4/P1 project lead and IPO Dictionary methodology committee coordination)

- Identify the rights and responsibilities for an AP planning project. (PMAG AP Coordinator)

- Template work break down structure for AP project planning. This should identify expectations on time, manpower resources, coordination requirements and necessary approvals. Sources for initial material include M. Mitchell's Development Plan for Application Protocols Mechanical Parts Production and S. Ryan's APDBE work breakdown structure. (PMAG AP Coordinator)

- WG4 projects, resource model projects and AP projects must maintain an accurate schedule which can be distributed by SC4. (WG4 Convenor, \& project leaders)

- WG4 should document how its priorities are established. (WG4 project leaders)

- Additional criteria to establish WG4 work priority; this should include the identification of AP capabilities needed by multiple industries. (PMAG AP Coordinator)

- Rules and procedures to defined on how gaps in the integrated resources are to be filled. The AP guidelines assume integrated resources are sufficient to satisfy the application requirements. A principal criteria for selecting the initial release APs was the existence of draft resource models. (i.e. there is no guidance on how new integrated resource models are initiated and added to a release of STEP)(WG4, PMAG)

- Rules and procedures to guide the development of AICs. How specific should AICs be? How to modify an existing AIC (P6, AP Integration Project) 
- Establish criteria is used to determine the commonality of requirements and the utility of existing AICs and APs for use by additional APs (AP integration)

\section{Improvements to existing documents identified:}

- Identify the minimum set of information needed to evaluate the scope of an AP. This is needed for the AP project approval process. (AP Qualification Manual \& AP Guidelines documents : Mark Palmer with submissions by WG4/P1).

a) $C D$ comments by member countries;

b) for AP project approval.

- Specify the AP components that are part of the submission for CD Comment ballot by PMAG and SC4. (AP Guidelines)

- Define the required contents of an ARM population section of the AP validation report (AP Qualification Manual and AP Guidelines)

- Define what constitutes an adequate ARM and AIM population coverage. (AP Qualification Manual and AP Guidelines)

- Document the IR version references used in the AIM. An AP should list the version ( $N$-number) of resource parts in mapping table and references in AP bibliography. (AP Guidelines)

- Define the required contents of the AIM compilation report. (AP Guidelines)

- Specify what EXPRESS compilers are approved for use in checking the correctness of the EXPRESS in the AIM (AP Guidelines \& AP Qualification Manual with guidance from WG6)

- Define how small of a market sector can AP target. An example is Navy shipbuilding; is this a large enough sector to warrant international standardization by ISO TC184/SC4 (AP Guidelines)

- Provide guidance on conformance requirements and test purposes to AP developers with respect to conversion between shape (geometric) representations. APs need to answer questions like: when is conversion is allowed; what test purposes are needed are needed to assess the result of a conversion, and what are the acceptance criteria. (AP Guidelines, Guidelines for the Development of Test Purpose )

- Define the required contents of an AIM population section of the AP validation report . (AP Qualification Manual and AP Guidelines)

- Define boiler plate text for integrated resource interpretation report (Supplemental Directives)

- Specify an appropriate introduction to the ARMAIM mapping table. Should material from the integrated resource interpretation report be used as the basis for the introduction to the ARM-AIM mapping table? (Supplemental Directives)

- Direct AP developers to document the intent of selected resource constructs in the integrated resource interpretation report (AP Guidelines)

- State that the AAM to ARM mapping report needs to be part of validation report (AP Qualification Manual and AP Guidelines)

- Require AP team to describe their information modeling expertise (AP Qualification Manual)

- Require proof that a structured approach to information gathering was used in developing the AP requirements. (AP Qualification Manual and AP Guidelines)

- Require proof that a method that ensures traceability of requirements from scope throughout AP development process was used. An AP should demonstrate that this has occurred; QFD is one possible technique. (AP Guidelines)

- Update the Supplement Directives to reflect the current AP development process. (Supplemental Directives)

- State how to assess the correspondance of AIM mapping table to test purposes. (AP Qualification Manual)

- Provide guidance on how to assess validity of requirements. In information gathering stage, domain experts may report planned not actual requirements. (AP Qualification Manual)

- State that to-be information requirements will not 
be allowed if the transformation from the as-is to the to-be cannot be produced. (AP Qualification Manual and AP Guidelines)

- Define how an AP is to demonstrate the correspondence between the scope, units of functionality, and requirements. The AP scope states the overall required functionality of the AP and Clause 4 states the information requirements. (AP Qualifications Manual)

- Identify what criteria is used to determine if an AP scope unique. (AP Guidelines)

\section{Required action external to WG4:}

- Develop consensus on criteria for committing resources to subsequent work which extends the functionality of STEP. (SPAG, IPO Steering

Committee; National TAG)

- Develop acceptance criteria for implementations of EXPRESS compilers. (WG6)

- Provide a list of approved and acceptance tested EXPRESS compilers to all STEP Part developers. (WG5 Express project)

- Decide on the inclusion of assertions in generating conformance test purposes. (WG6)

- Develop procedure for review and approve AP test purposes. (WG6)

- Define a procedure for validating EXPRESS compilers. (WG6)

Mark Palmer presented some of these recommendations at the Wednesday PMAG.

\section{AP Qualification Manual additional criteria proposed}

For validation of the AP requirements clause:

- Proof that there was a trained and experienced information requirements staff.
- Proof that the project employed a structured information gathering technique. (The AP project should submit questionnaire or other documented method for group 1 review.)

- Demonstrate the AP can support a broad industrial application by obtaining actual data from multiple enterprises and more than one country and populating both the ARM and AIM with it.

- Document reviews of the capabilities within existing commercial systems which support all or part of the application scope.

- Proof of the existence of a plan that was used for coordinating the requirements analysis.

Are the requirements within the AP scope?

- Document with the external reviewer evaluation, the use a structured method.

- Check that the AP project has established priority within the AP framework.

Further Discussion Required:

If an AP goes beyond existing practices and provides a to-be process model, how should this be validated?

Should the ARM-AIM mapping require that transitive closure be proved?

Should the mapping structure provide the exactness that would allow the ARM to be reverse engineered from the AIM as a verification technique?

\section{Traceability of Requirements}

- The lack of traceability can be tied to the need to improve the guidance on how an AP is to document that the AP development process was followed.

- Some flexibility should be allowed in how to demonstrate traceability. Equivalent approaches are likely. We lack experience to select any specific one as the best method to use. Especially for the normative elements of an AP, we need to establish what the minimum requirement is.

- All requirements should be traceable to an ARM 
object. If it cannot be traced then it is probable that the resulting AP cannot satisfy this requirement.

- Develop usages tests based on expert interviews and existing application system analysis and expand the constraints uncovered by these into AIM reference path queries. These frequently reflect important aspects which are necessary to satisfy application requirements.

- Extend the current mapping table structure to eliminate the following limitations

a) the current structure is unable to express relationships including cardinality,

b) ARM many-to-many relationships are not cleanly represented in the mapping table, and

c) constraints in the AIM which have significance in the ARM are not represented 


\section{REQUIREMENTS FOR AP VALIDATION \& THE AP VALIDATION REPORT}

\section{Larry McKee}

Our group discussed the following topics:

documenting the application interpretation; the extent that an AIM should be test populated with data; assessment of conformance requirements and test purposes. An AIM should be test populated with data and that population should be provided in a report that at a minimum should access the degree of coverage of the population, meaning how much of the AIM did you actually populate. The minimum data used in the population should be the same part used to populate the ARM. Another piece of documentation for the AIM is a list of the numbers of the integrated resources Parts that were used to make the AIM. The AP document should state exactly what versions of the Parts were used in building the AIM so that there is no doubt of the version used in the AIM.

Another requirement is a compile of the EXPRESS for the AIM. The results of that compile are to be provided in documentation. For the group 3 evaluation of conformance requirements and test purposes, especially for test purposes it doesn't make sense to evaluation them by themselves. They need to be evaluated against the AIM to make sure all the required test purposes are there. For conformance requirements, the test purpose should indicate which of the AP's various implementation levels they apply to.
One of the validations we talked about, but we did not discuss how to document, was determining what the conformance requirements for the implementation level are and that it should map into a closed set of entities. When you conform to this implementation level, it uses a fixed and closed set of entities that you must conform to.

\section{Documenting the Validity of an $\mathrm{AP}$}

One form of documentation should be the results of the ballot of the scope, AAM and ARM along with comment resolutions. Another part of the report should be the ARM population report with a coverage analysis which identifies how much of the model was populated. Furthermore, it describes why things were not populated. The report should list the $\mathrm{N}$-numbered integrated resource parts used in the AIM compilation report. The AIM population report should be similar to the ARM population report; it should show that at least the ARM test part was used in the population and the results of the population should be described. Test purpose to AIM construct mapping report shows that for every enumerated construct in the AIM you have a test purpose that exercises all optional and enumerated values. The integrated resource interpretation report describes how the interpretation was done and where the mapped elements came from. There was a proposal for WG6 to perform test purpose review and that the results of that review from WG6 the AP 
developers will be responsible for covering any comments that WG6 had against the test purposes and how they were resolved. On the AAM to ARM mapping report, assuming the use of IDEF 0 for your AAM, each one of the inputs and outputs should be mapped into ARM data constructs. It won't be one-to-one mapping but it should show that the in-scope inputs and outputs are covered. Another part of this document essentially takes what has been called complex test purposes and shows how they are used against the AIM to produce the data which satisfies the input and output requirements. Those are the things we have identified in the model validation methodology. The fundamental thing we need to agree on with respect to AP validation is to get an up-front validation of the scoping requirements to eliminate the problems we have seen in all of the APs up to now with requirements extensions and contractions. The AP projects must make sure they have an agreed upon set of requirements.

The other thing discussed was where the ballot comments came from. Industry expert reviews tend to be done within the confines of your group, within U.S., European, or Asian, etc.; they tend to not extend into the entire ISO arena. Using the ballot mechanism would force an international review of scope and requirements. This ballot would not be an approve or rejection; the intent is to just verify that none of the requirements have been left out. The problem we have experienced, is that if you send a document out to all the $\mathrm{P}$ member countries for comment and not for ballot, we do not get any response. If we send the AP scope and requirements out for ballot, we will probably get some meaningful responses because the $\mathbf{P}$ member countries must reply.

Question and answer period. 


\section{References}

[1] Palmer, M., Gilbert, M., and Anderson, J.;

Guidelines for the Development and Approval of STEP Application Protocols, ISO TC184/SC4/WG4.

[2] STEP Application Protocol Qualification Manual, ISO TC184/SC4/WG4/P5 AP Guidelines and Framework Project, Owner: Palmer, $\mathrm{M}$.

[3] Guidelines for the Development of Test Purposes, Version 1, ISO

TC184/SC4/WG6, Owner: Fowler, J.

[4] Danner, W. and Yang, Y., STEP

Development Methods: Resource Integration and Application Interpretation, ISO TC184/SC4/WG5 .

[5] The EXPRESS Usage Guide, ISO TC184/SC4/WG5 EXPRESS Methodology Project Draft, Owner. Brett, S.

[6] STEP Part Qualification Procedures, P1: Qualification Project, ISO C184/SC4/WG4, Document N31, Owner: Burkett, W.

[7] Jorgenson, B., Tyler, J., Williams, A., and Zimmermann, J.; Model Qualification Criteria for PDES/STEP, Revision 1.2, IGES/PDES Organization, Dictionary Methodology Committee

[8] Mitchell, M., A Proposed Methodology for STEP A pplication Protocol Validation, NISTIR 4683. 


\section{Attendees}

Jon Aas

5 Coles Lane

Oakington

Cambridge CB4 5BA

United Kingdom

Diane Allen

One Northrop Ave

Organization 566/AV

Hawthorne, Califomia 90250

422-9777@mcimail.com

Allison Barnard NIST
Bldg 220, Room A127
Gaithersburg, Maryland 20899
barnard@cme.nist.gov
John H. Barnes Air Force MANTECH
USAF
Building 653, WL/MTIB
Wright Patterson AFB, Ohio 45433
513) 255-7371
barmesih@mlgate.ml.wpafb.af.mil

\section{Steve Brett}

CADDETC

Amdale House

Headingley

Leeds LS6 2UU

United Kingdom

Tel: +4453230500

Fax: +4453230448

steve@uk.ac.leeds.leva

Bill Burkett

PDIT

6433 Topanga Canyon Blvd Box 443

Canoga Park, California 91303

burkett@girtab.usc.edu

\section{Pat Chavez}

Sandia National Lab

NIPDE/NIST

Bldg 220, Room 317

Gaithersburg, Maryland 20899

Bill Conroy

NIST

Bldg 220, Room A127

Gaithersburg, Maryland 20899

conroy@cme.nist.gov
Cita Furlani NIST

Bldg 220, Room A127

Gaithersburg, Maryland 20899

furlani@cme.nist.gov

Jeane Ford NIST

Bldg 220, Room A127

Gaithersburg, Md. 20899

Pete Lazo Newport News

Dept E20, Bldg $600 \quad$ Shipbuilding

4101 Washington Ave.

Newport News, Virginia 23607

Jochen Haenisch $\quad S I$

PO Box 124, Blindern

$\mathrm{N}-0314$

Oslo, Norway

haenisch@si.no

Peter Kruse

IMW TU Clausthal

Robert-Koch Str.

3392 Clausthal-Fellerfeld

Germany

kruse@sunslc;lmw.TU-Clausthal.DE

Doug Martin

NASSCO

PO Box 85278 MS05

San Diego, California 92186-527?

Compuserve 70412,3036

Larry McKee

IBM

9500 Godwin Drive

Attn: 102/072

Manassas, Virginia 22110

374-9667@mcimail.com

Mary Mitchell

NIST

Bldg 220, Room 127

Gaithersburg, Maryland 20899

mitchell@cme.nist.gov

Kurudi Muralidhar Murali Industrial

PO Box 1485 Technology Institute

Ann Arbor, Michigan 48106

313) 769-4337

khm@iti.org 


\begin{tabular}{|c|c|}
\hline $\begin{array}{l}\text { Constance Orogo } \\
1450 \text { Scalp }\end{array}$ & $\begin{array}{l}\text { Metal Working } \\
\text { Technology }\end{array}$ \\
\hline \multicolumn{2}{|c|}{ Johnstown, Pennsylvania 15904} \\
\hline $\begin{array}{l}\text { Jon Owen } \\
\text { University of Leeds } \\
171 \text { Woodhouse Lane } \\
\text { Leeds LS2 3AR } \\
\text { United Kingdom } \\
\text { jon@uk.ac.leeds.leva }\end{array}$ & CADDETC \\
\hline $\begin{array}{l}\text { Mark Palmer } \\
\text { Bldg 226, Room B306 } \\
\text { Gaithersburg, Maryland } \\
\text { palmer@enh_nist.gov }\end{array}$ & $\begin{array}{l}\text { NIST } \\
0899\end{array}$ \\
\hline $\begin{array}{l}\text { Haidee Rapacki } \\
12500 \text { N Washington } \\
\text { Denver, Colorado } 8024\end{array}$ & $\begin{array}{l}\text { Auto-trol Technology } \\
2404\end{array}$ \\
\hline
\end{tabular}

Kent Reed NIST

Bldg 226 Room B306

Gaithersburg, Maryland 20899

Guy Renfro Boeing

Boeing Defense \& Space

PO Box 3999, MS 80?

Seattle, Washington 98124-249

renfrog\%net.A1@ATC.Boeing.com

Sandy Ressler NIST

Bldg 220 Room A127

Gaithersburg, Maryland 20899

ressler@cme.nist.gov

\section{Bill Russell AF Wright Lab}

USAF

Bldg 653/WL/MTI, Room 218

Wright Patterson Air Force Base, Ohio 45433

Steve Ryan

1 Neumann Way

Cincinnati, Ohio 45215

369-6141@mcimail.com
Nigel Shaw

British Aerospace

$\mathrm{BAe}$

CTU, Broughton.

Chester, CH4 ODR

UK

4340247@mcimail.com

Bob Street

Lockheed

Z0199 Dept 730

86 S. Cobb Drive

Marietta, Georgia 30063

5036455@mcimail.com

Roger Stumps

Boeing

PO Box 7730

M/SK46-12

Wichita, Kansas 67277

rls0028@KS.Boeing.Com

Anne Williams

M.C. 6800950

13736 Riverport Drive

Maryland Heights, Missouri 63043

John Zimmerman

Allied-Signal KCD

2000 E 95th St.

D/878 MS FB-22

Kansas City, Missouri 64131 


\section{Annex}





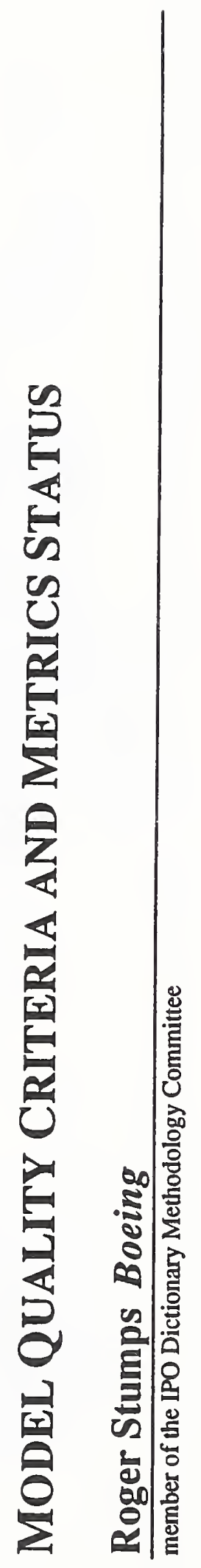




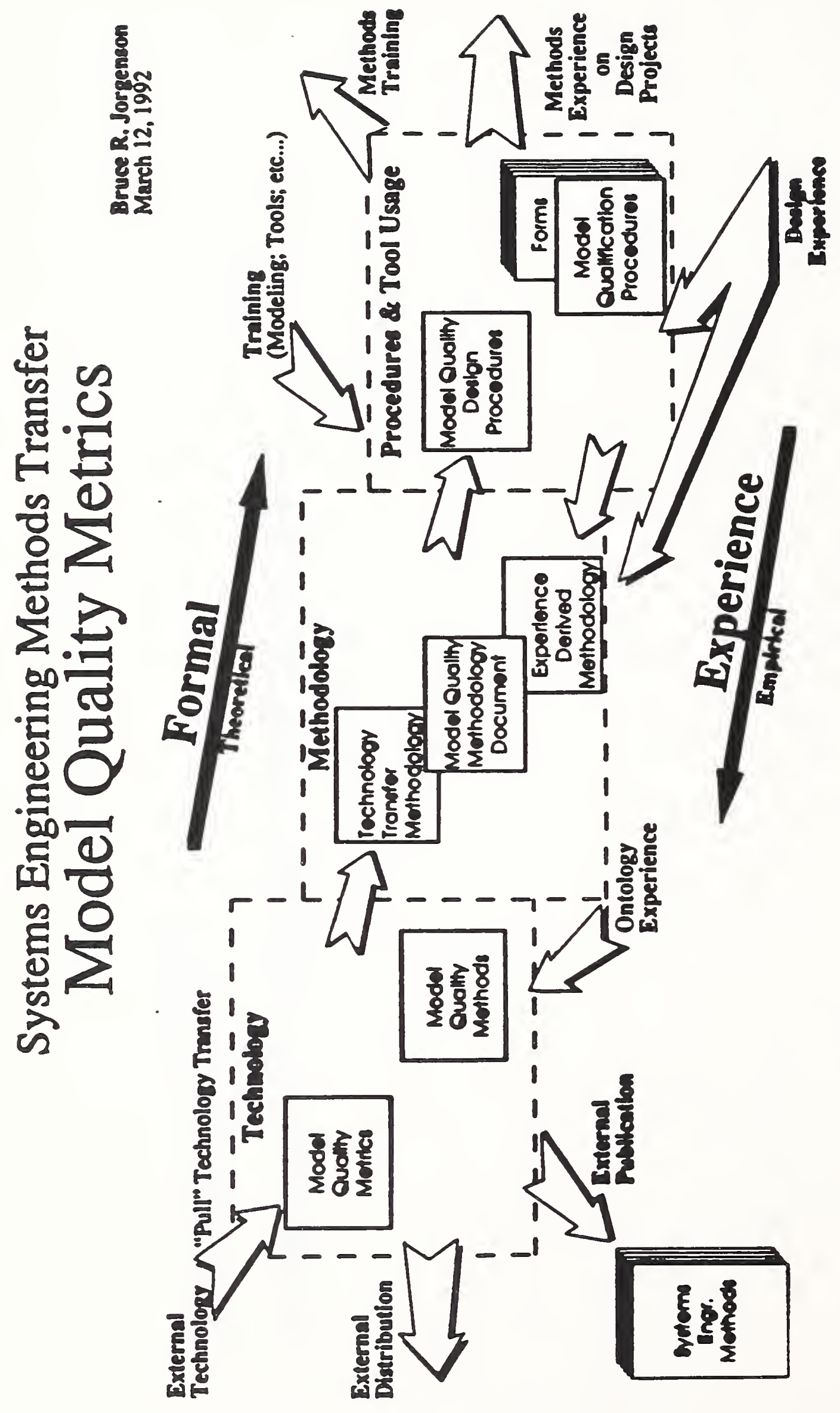




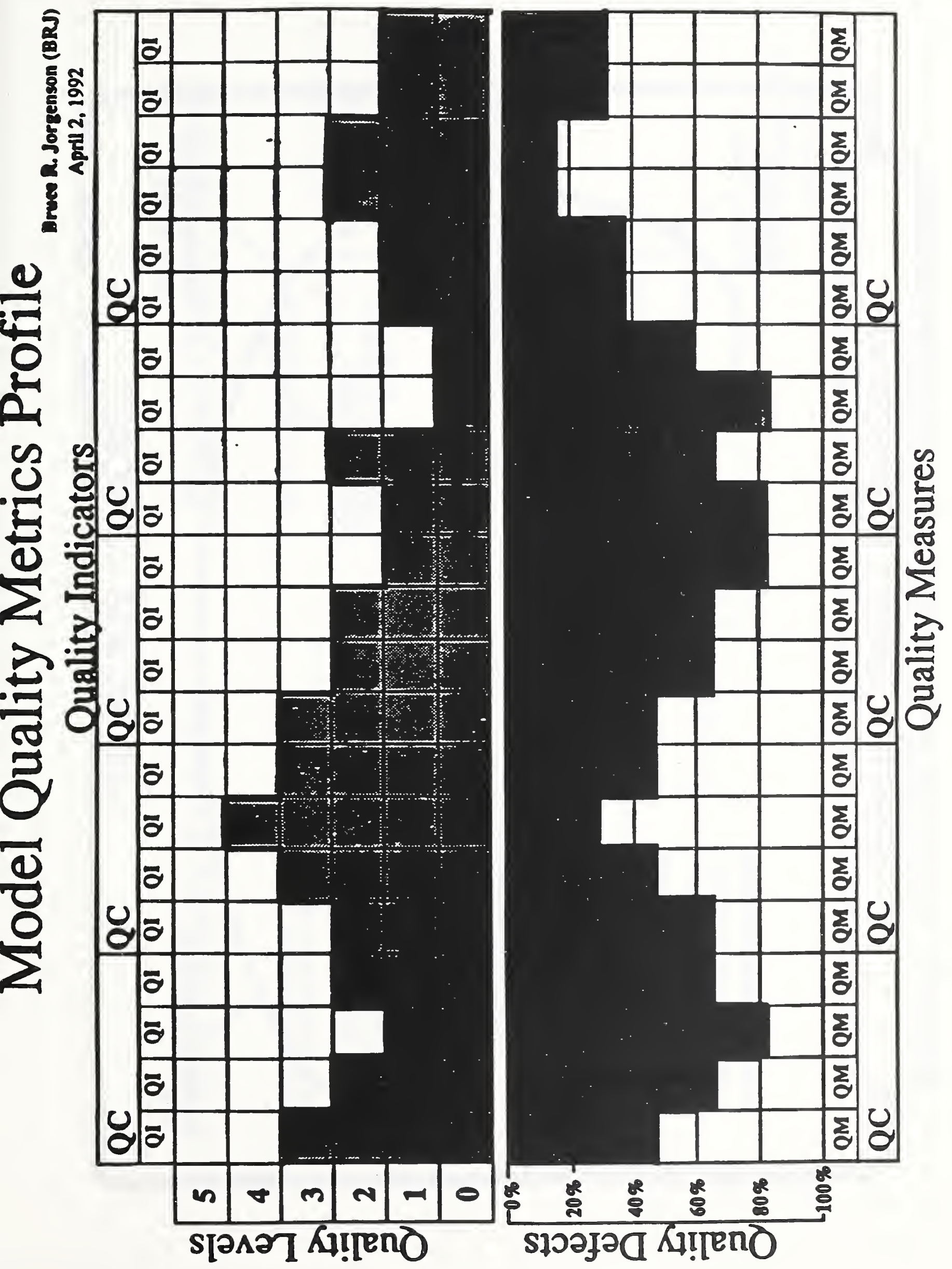




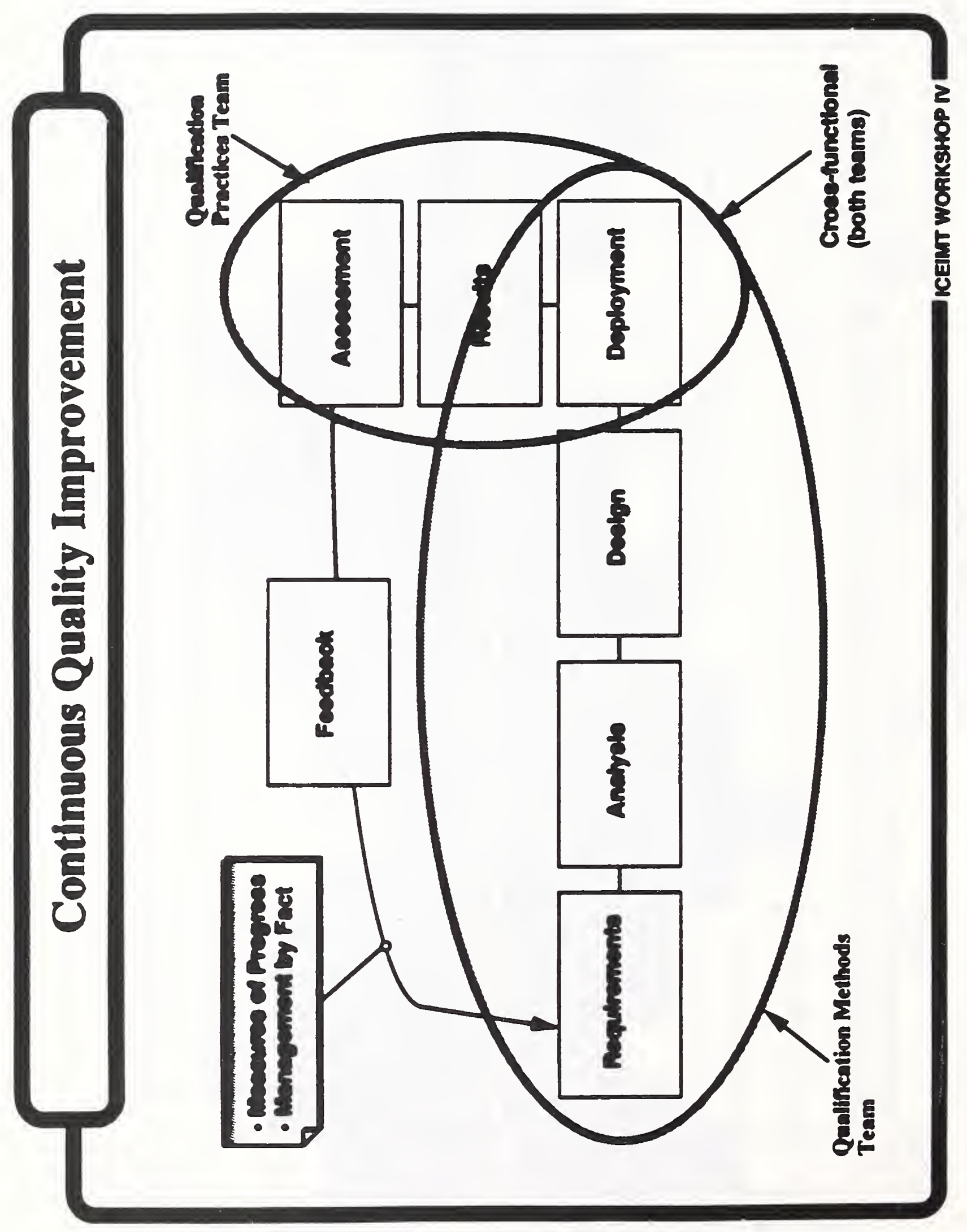




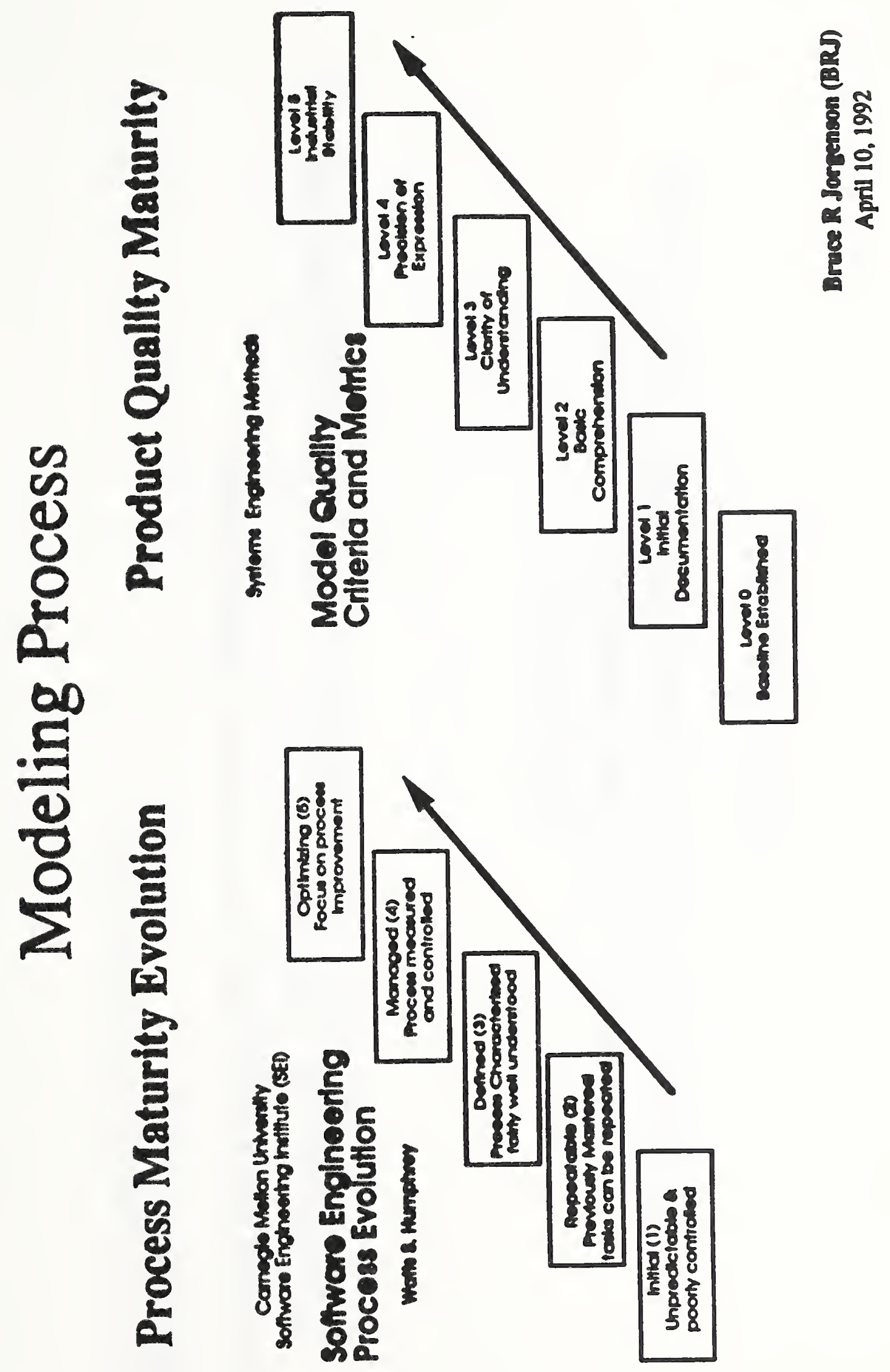




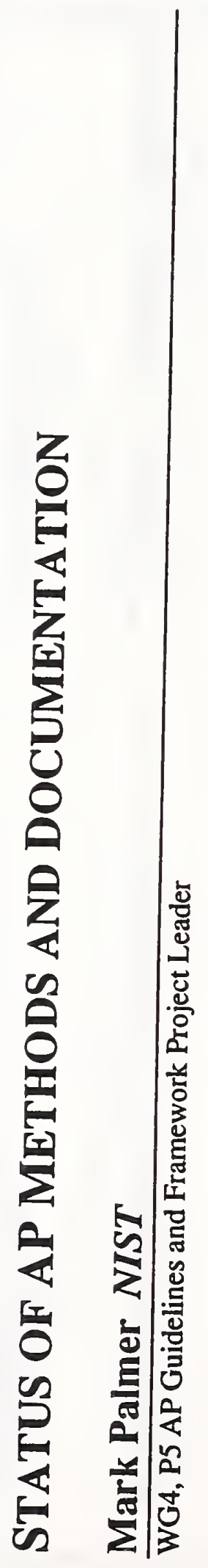




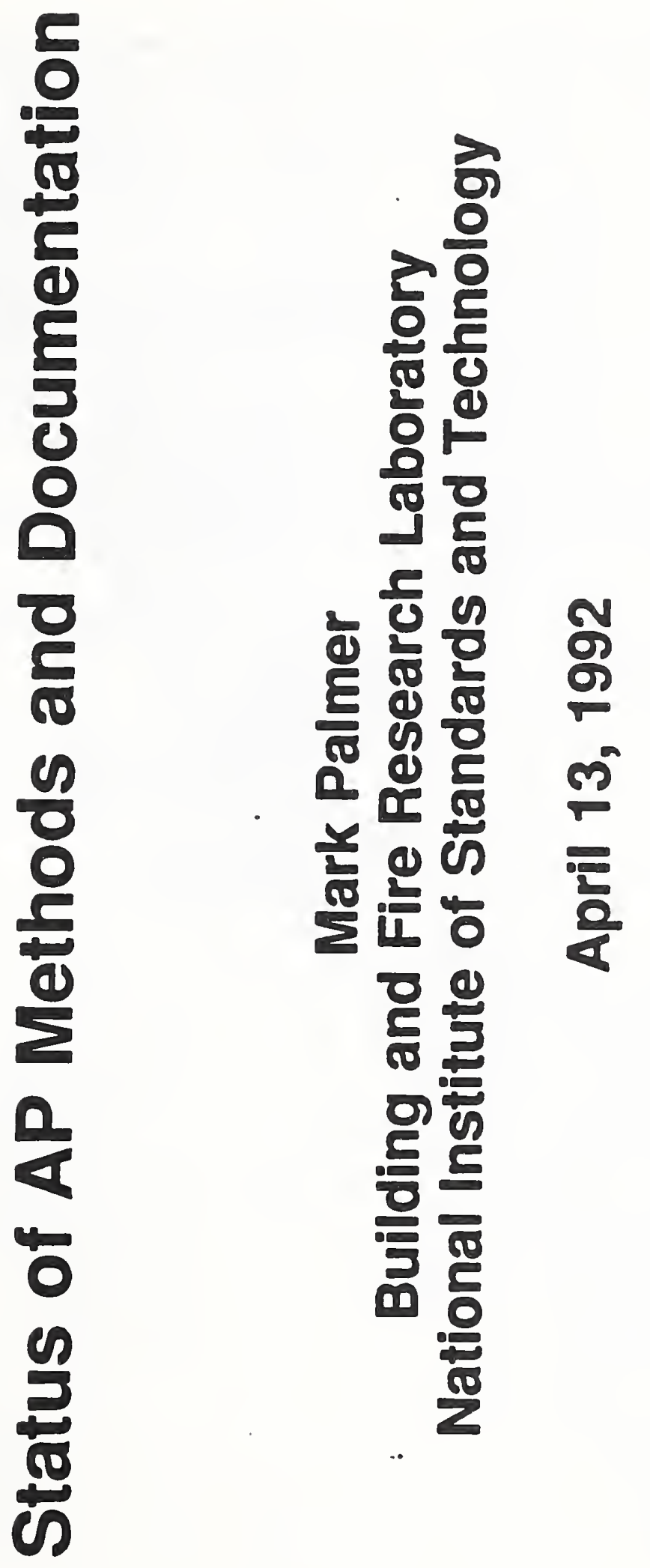




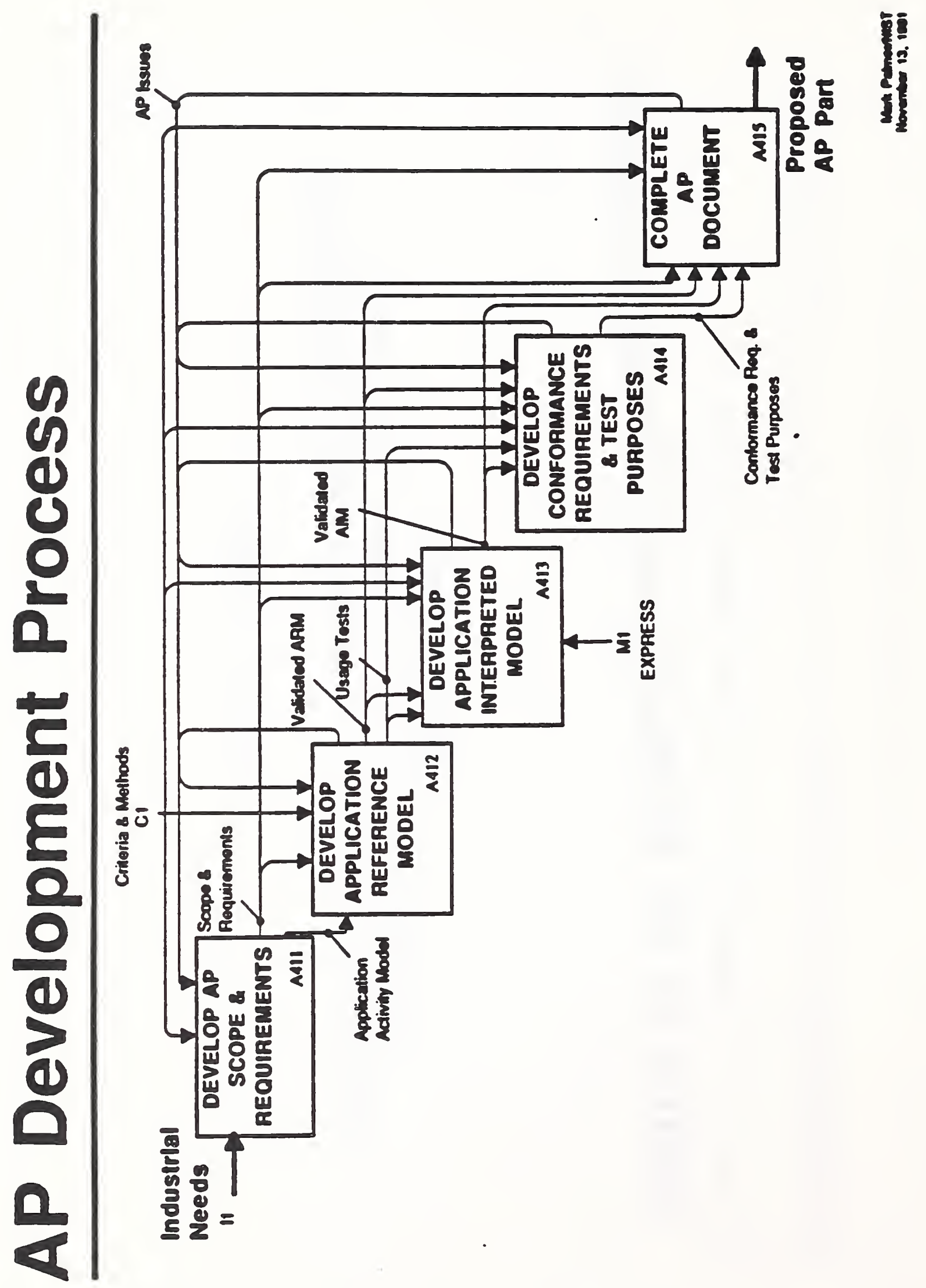




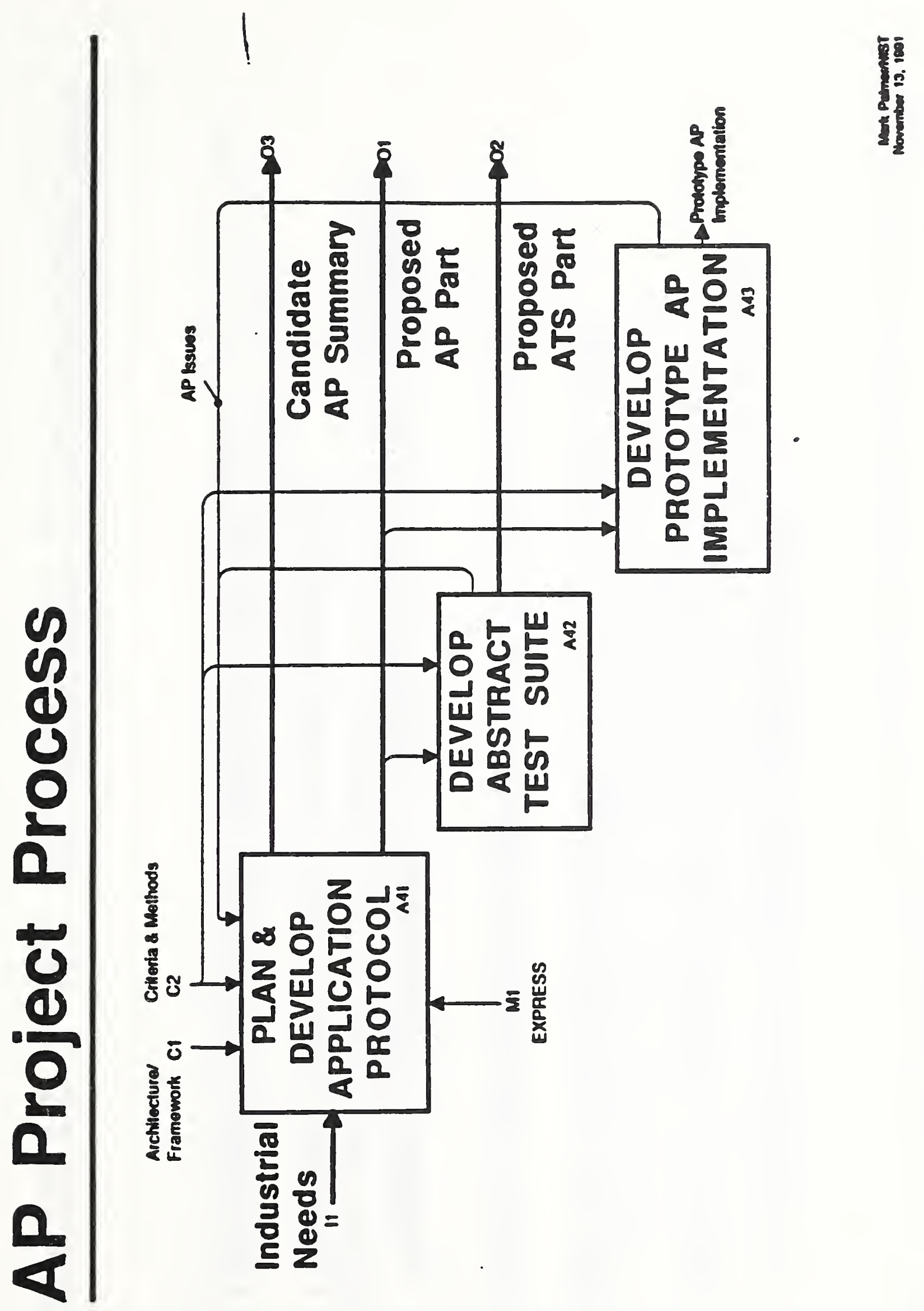




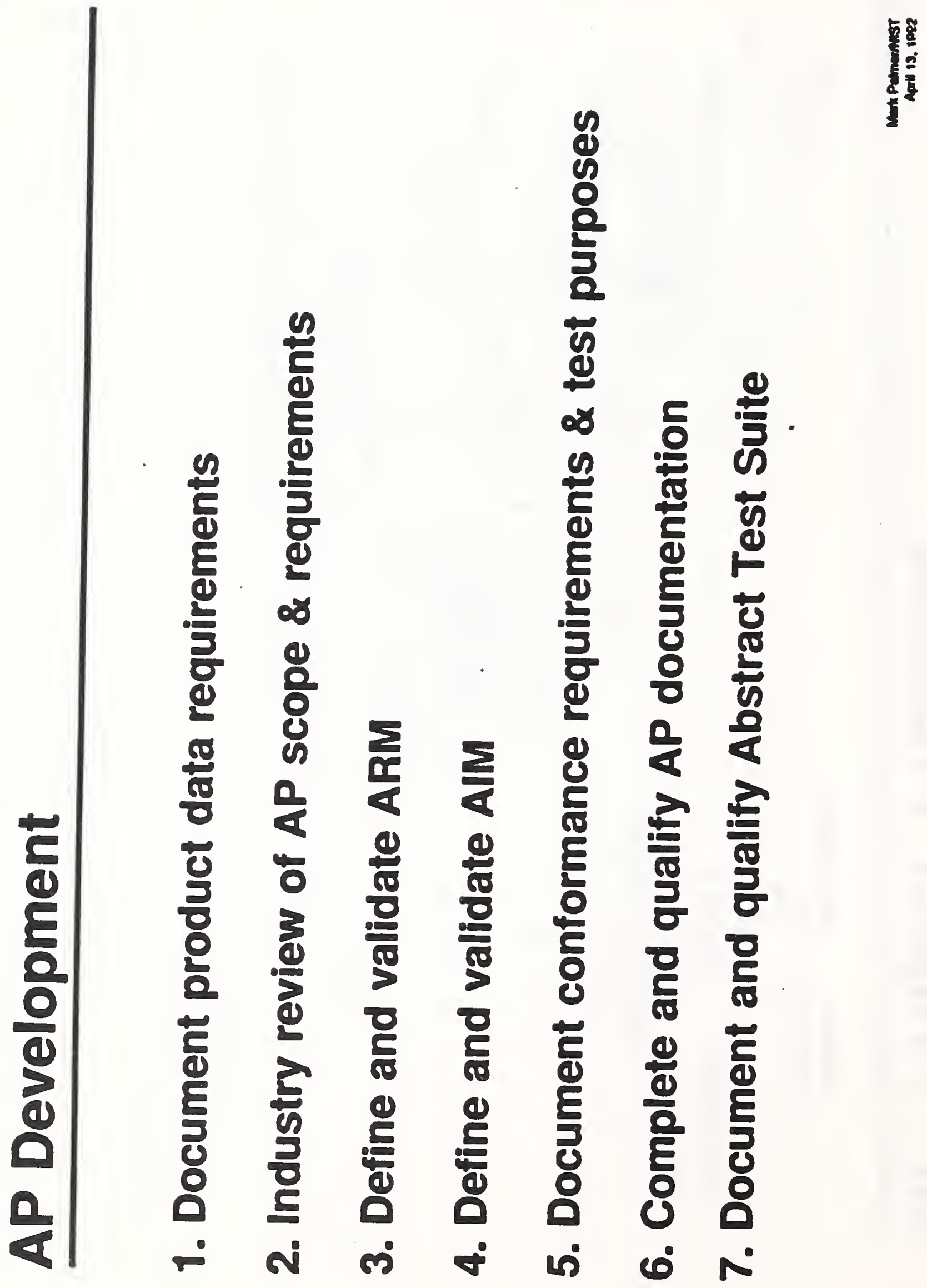




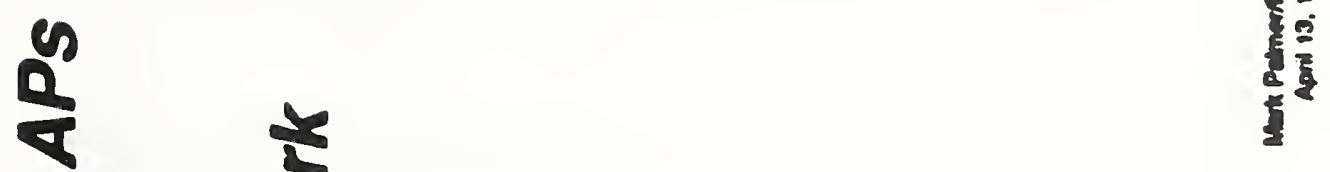

$\frac{0}{0}$

D)

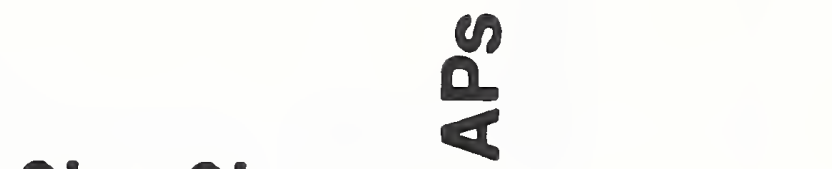

(1)

4020

05

N

$\frac{1}{4}$

3

200

98

820

Q1 4

$\frac{0}{11}$

200

$\sum_{20}^{\infty}$

020

2

$\infty 2$

(1)

(b) 80

\&

$0 \%$

Q 1

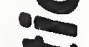

$\rightarrow 2$

( 60

60

(1) $\frac{1}{\text { a }}$

05

(a) 02

(1) 2

a.

II

85

a) 2

5

$\infty 2$

20

8

62

(2)

() 18

(1)

\& 00 क 0

\& की के के

$E_{0}^{\circ}$

0

\& 200

a) $\pi$

EU 00

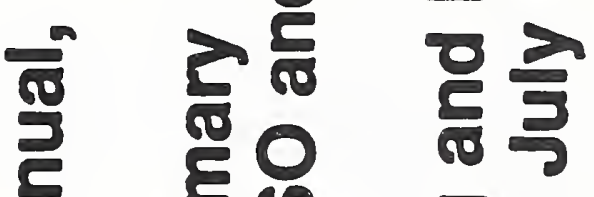

$6 \%$

(2) 1

8

6

\%

$\Leftrightarrow 2$

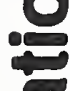

05

E

80

10

(2)

50

(i) 2

(1)

01

00

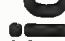

든



600

5

8

중

(1)

는

0

는

- 5

a 0

U1

a 20

잉

00

$\frac{1}{2}=$

( 2

55

(1) 0

룽

8

in

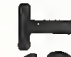

$-5$ 


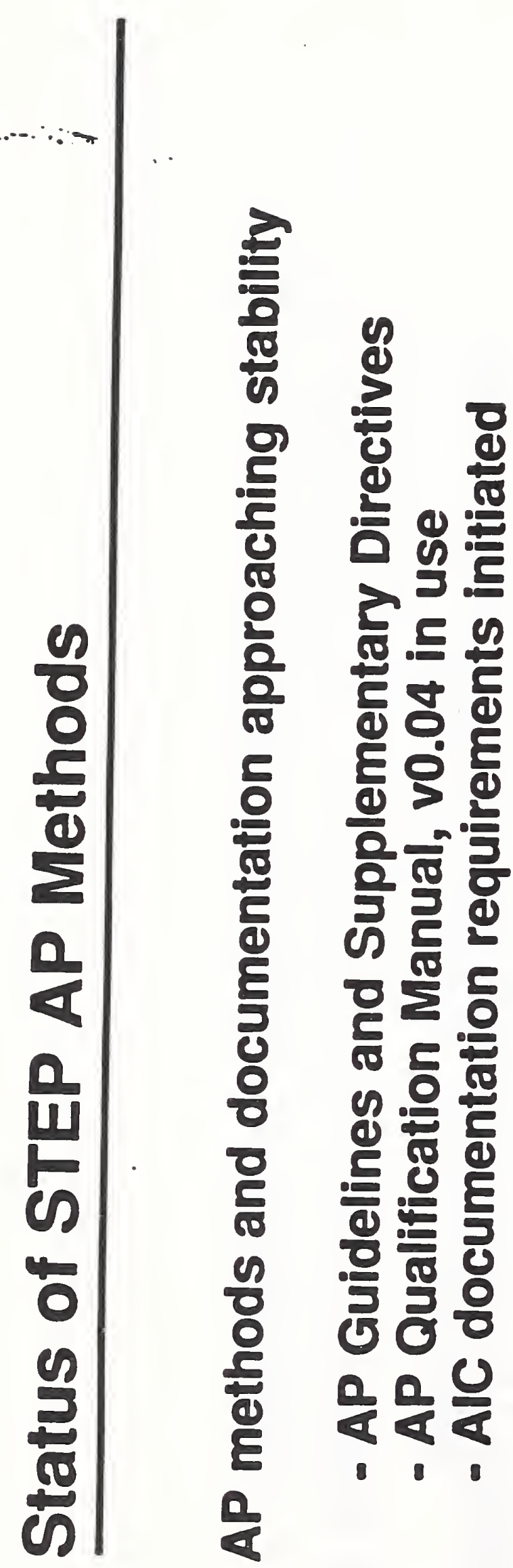

$\stackrel{n}{2}$

$\frac{5}{5}$
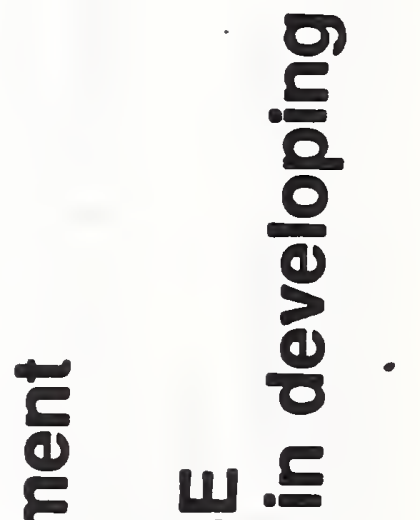

$\frac{5}{x}$

o

d

동

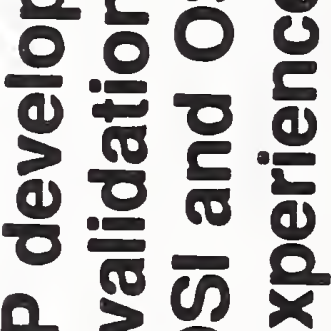

< 0 ब

ㅎํㅇ은 흥

응오을을 응

엉당

-

จ

으

(1) 0 :

\%

은

$\overline{8}$

.0ํㅡㄹ

엉 을응 응

.트를 


\section{AP Development and Validation Issues}

1. Verification and international consensus of $A P$ scope and requirements

- process and procedures defined

2. ARM development and validation

- specificity of industry semantics and detail (granularity) of ARM

- rules for defining "units of functionality" (UoFs)

- verification of UoFs

- role of "functional building blocks" to extend core ARM

3. AlM development and validation

- ensuring traceability from the AP requirements to AIM to ATS

- rules and tools for preparing the AlM long form from short form

- how to assess the AIM short form, how to assess the AIM long form

- procedures for building and validating reusable AICs

4. Development and validation of test purposes, abstract test suite (ATS), and executable test cases

- procedures for deriving test groups and test purposes

- required format and granularity of conformance test purposes

- consensus on the value and role of basic test purposes and complex test purposes

- how to assess the conformance requirements and test purposes

- viability of using the same test purposes and conformance requirements to generate an ATS for an exchange file implementation and an ATS for a distributed databases implementation

- rules and procedures for producing an ATS

5. AP development and documentation

- expanded mapping table: understandability and simplify the enumeration of test purposes

- enforcing validation of APs and documenting the results

- inclusion of standardized queries for obtaining the ARM concepts from the AIM

- contents and role of AP usage guide 


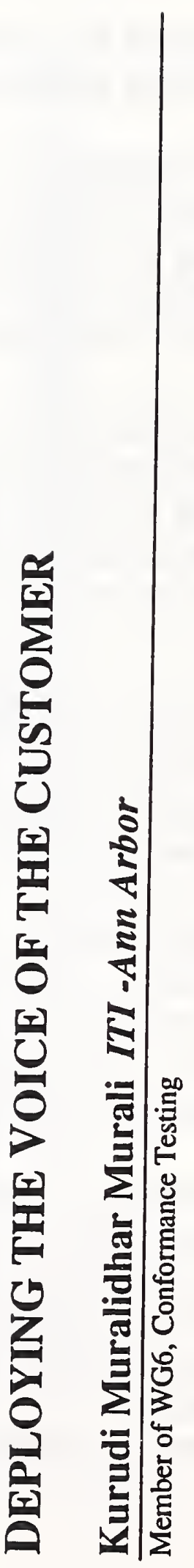




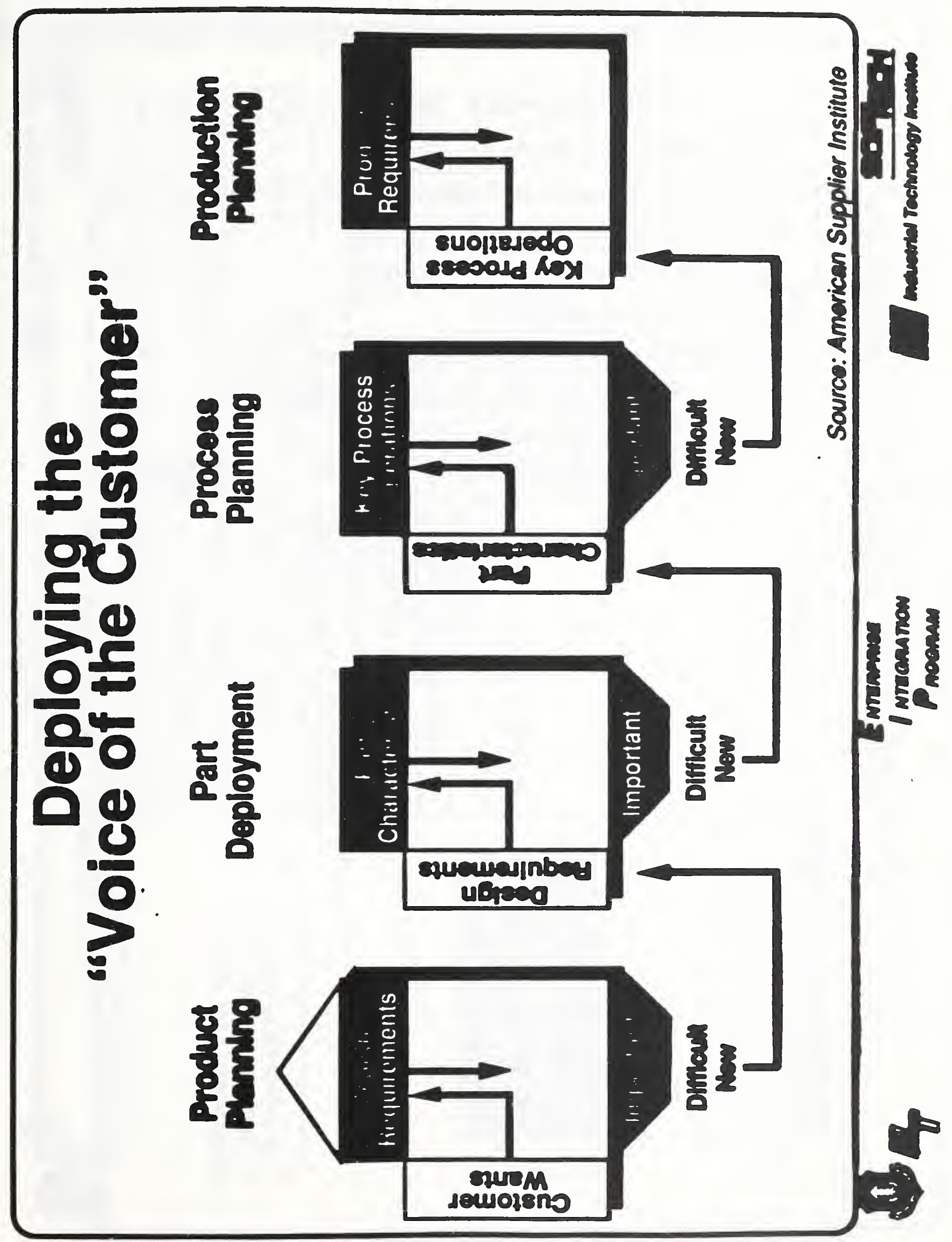




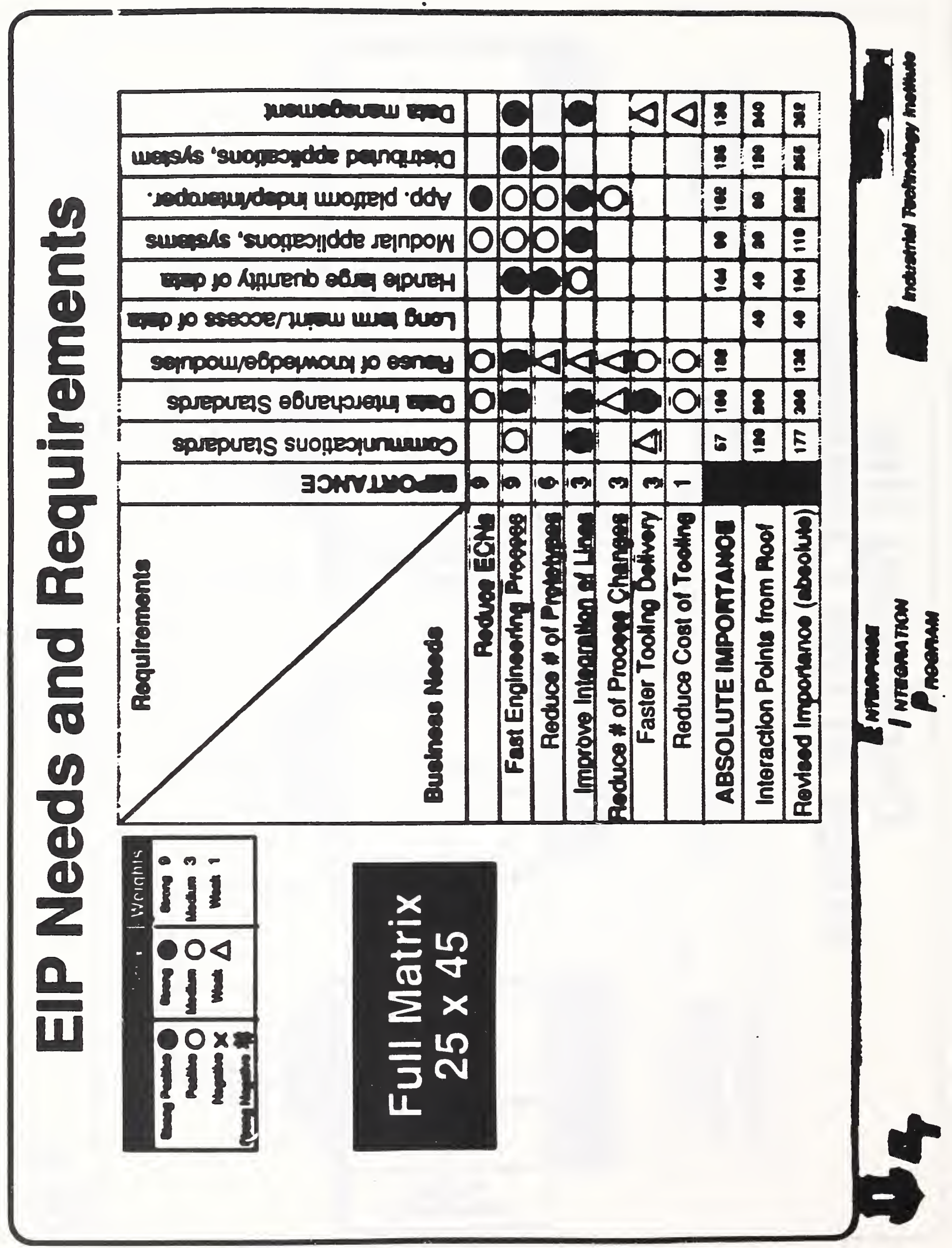




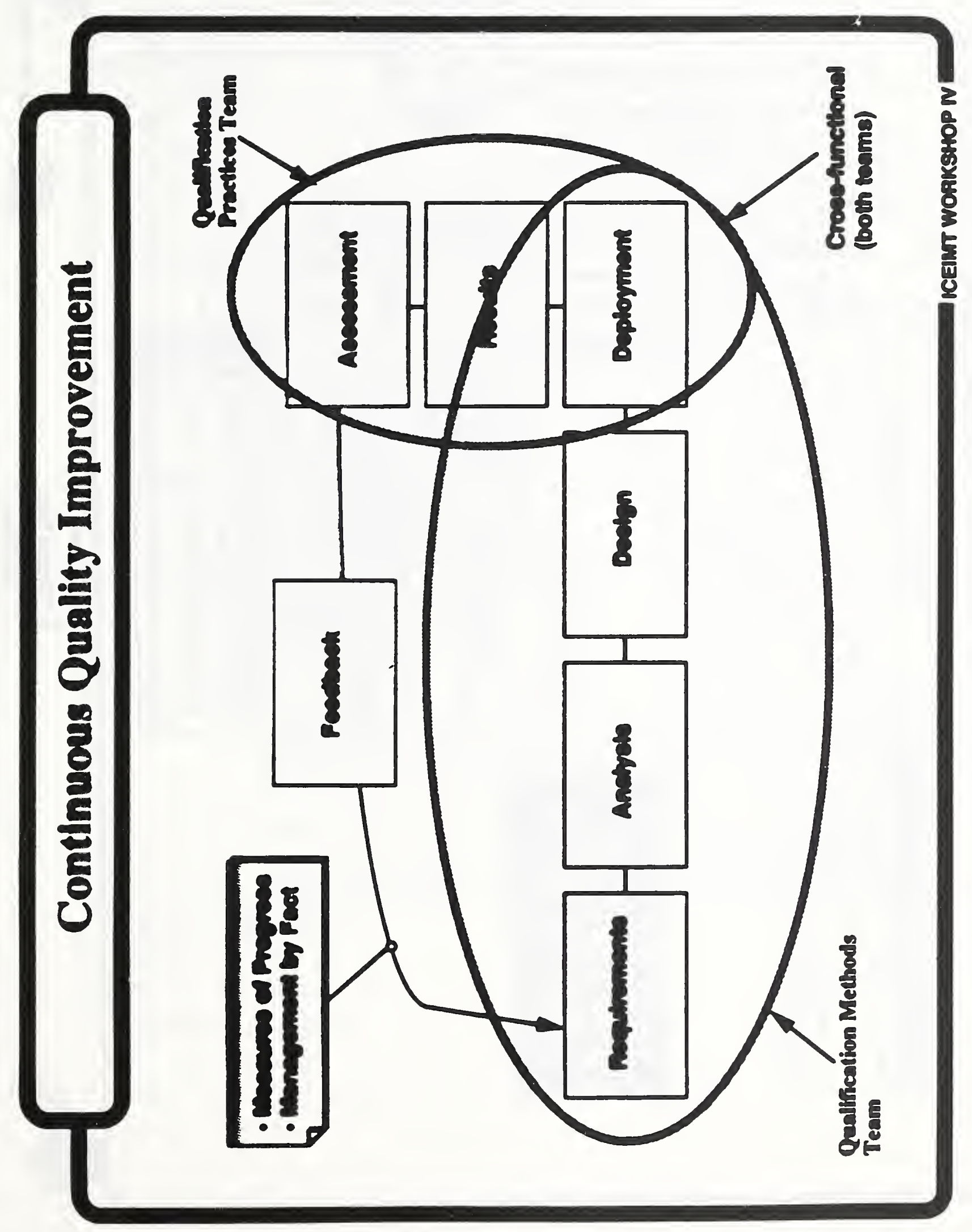




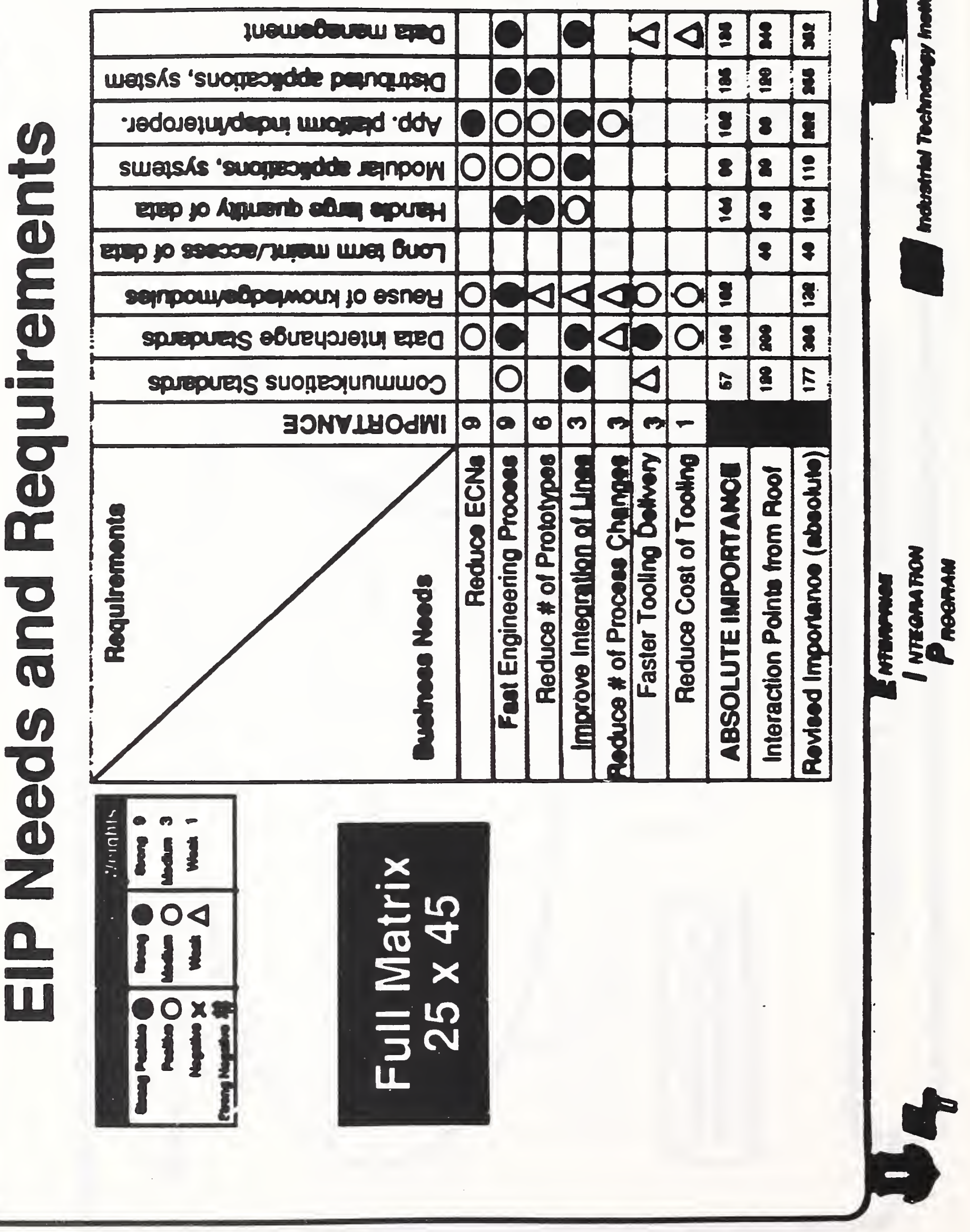




\begin{tabular}{|c|c|c|c|c|c|c|c|c|}
\hline \multicolumn{2}{|c|}{ REOUIREMENTS } & 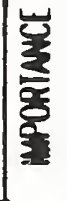 & 剀 & 영 & $\stackrel{z}{\underline{J}}$ & $\frac{\omega}{\tilde{J}}$ & 능 & \pm \\
\hline \multirow{5}{*}{ NSTANTIATION } & EXPRESS Compotible & 9 & (2) & 0 & & & & \\
\hline & Declare and Volves to ExpRESS Tyoes & 9 & (?) & $\mathrm{O}$ & $\triangle$ & $\triangle$ & & $\mathrm{O}$ \\
\hline & Models Must Be Self Conteined & 6 & (2) & (2) & & & & (2) \\
\hline & Handle EXPRESS expressions & 6 & (2) & 0 & $\triangle$ & $\triangle$ & & (2) \\
\hline & Sove and Retrieve Models & 6 & $\Delta$ & (2) & $\triangle$ & & $\triangle$ & 0 \\
\hline \multirow{2}{*}{ Monipulation } & Șend Commands & 16 & & O & O & $\triangle$ & (2) & 0 \\
\hline & Describe Commonds & .6 & & 0 & $\triangle$ & $\triangle$ & $\triangle$ & 0 \\
\hline \multirow{7}{*}{ Testing } & Specify Admin Information & 16 & & $\triangle$ & ○ & $\triangle$ & $\triangle$ & $\mathrm{O}$ \\
\hline & Test Cose Administration & 3 & & $\triangle$ & ○ & $\triangle$ & $\triangle$ & 0 \\
\hline & Specify Test Result Evaluation & 9 & & $\triangle$ & (2) & $\triangle$ & $\triangle$ & $\Delta$ \\
\hline & Reoch o Verdict & 9 & & $\Delta$ & (2) & $\triangle$ & $\triangle$ & $\Delta$ \\
\hline & Execute Test Bosed on Results & 16 & & $\triangle$ & (2) & $\triangle$ & 0 & $\triangle$ \\
\hline & Reoch Inside the Model & 16 & $\triangle$ & (2) & $\triangle$ & & & $\triangle$ \\
\hline & Specify Comporison to Reference & 9 & & 0 & (2) & $\triangle$ & O & $\triangle$ \\
\hline \multirow{8}{*}{ High Level } & Well Defined & 6 & 0 & $\triangle$ & 0 & 0 & (6) & (2) \\
\hline & Humon Understancoble & 3 & 0 & $\triangle$ & () & 0 & $\triangle$ & $\triangle$ \\
\hline & Mochine Reodoble & 9 & 0 & () & 0 & (2) & 0 & (2) \\
\hline & Simple & 3 & $\triangle$ & $\triangle$ & 0 & 0 & 0 & $\mathrm{O}$ \\
\hline & Mature & 3 & & $\triangle$ & 0 & (2) & () & (6) \\
\hline & Extensible & 6 & $\triangle$ & 0 & 0 & $\triangle$ & $\triangle$ & C \\
\hline & Supported by Tests and Compilers & 9 & & 0 & 0 & () & (2) & (2) \\
\hline & Support Executable Tests Automation & 9 & & & $\Delta$ & 0 & $\triangle$ & 6 \\
\hline \multicolumn{2}{|r|}{ ABSOLUTE IMPORTANCE } & & $\frac{\Omega}{2}$ & $\equiv$ & $\tilde{n}$ & $\mathscr{3}$ & $\vec{S}$ & $\widetilde{\widetilde{\Sigma}}$ \\
\hline & RELATIVE IMPORTANCE & & $\underline{E}$ & 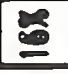 & $\mathscr{8}$ & 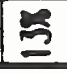 & $\Xi$ & $1 \approx$ \\
\hline
\end{tabular}

\begin{tabular}{|cc}
\hline MATRIX & WEIGHTS \\
Strong 0 & 9 \\
Medium 0 & 3 \\
Wook & 1 \\
\hline
\end{tabular}




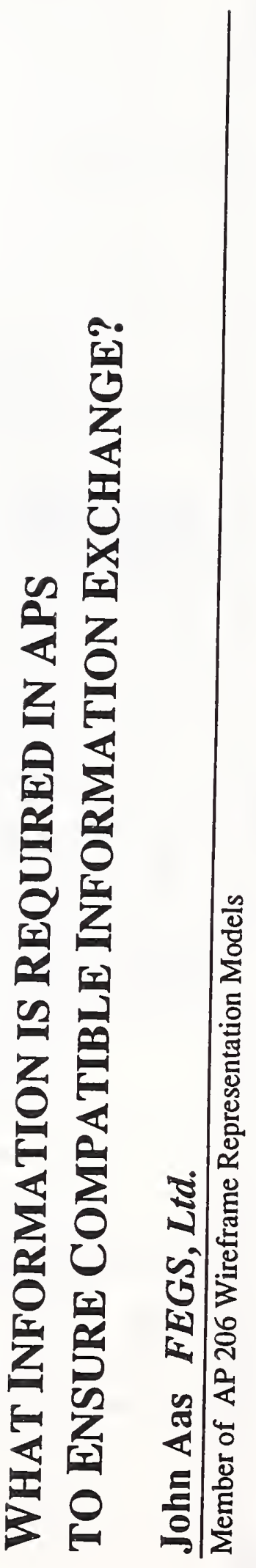



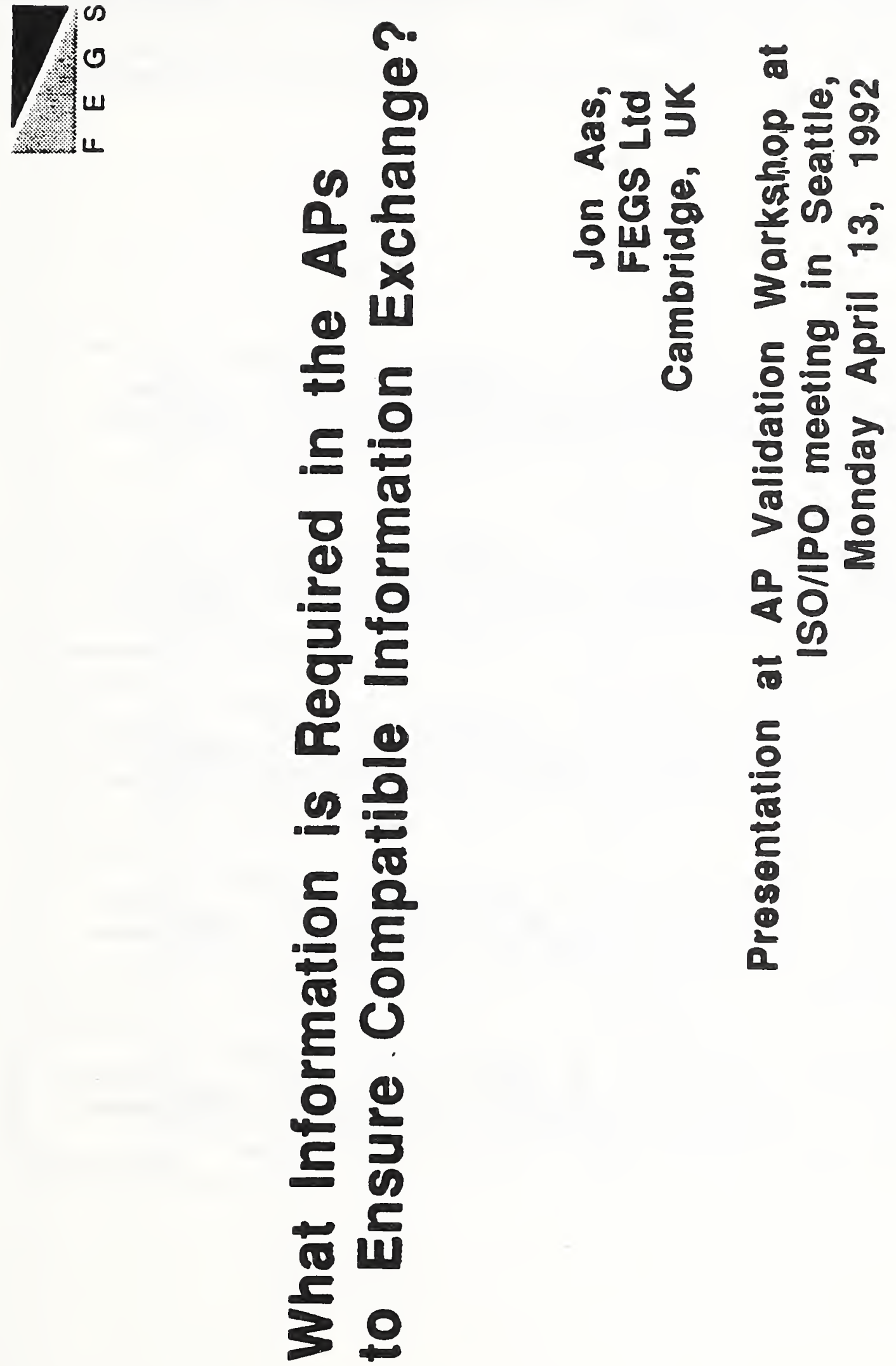


\section{ESPRIT CIM PROJECT 2195
FAMexchange architecture:
separate processors}
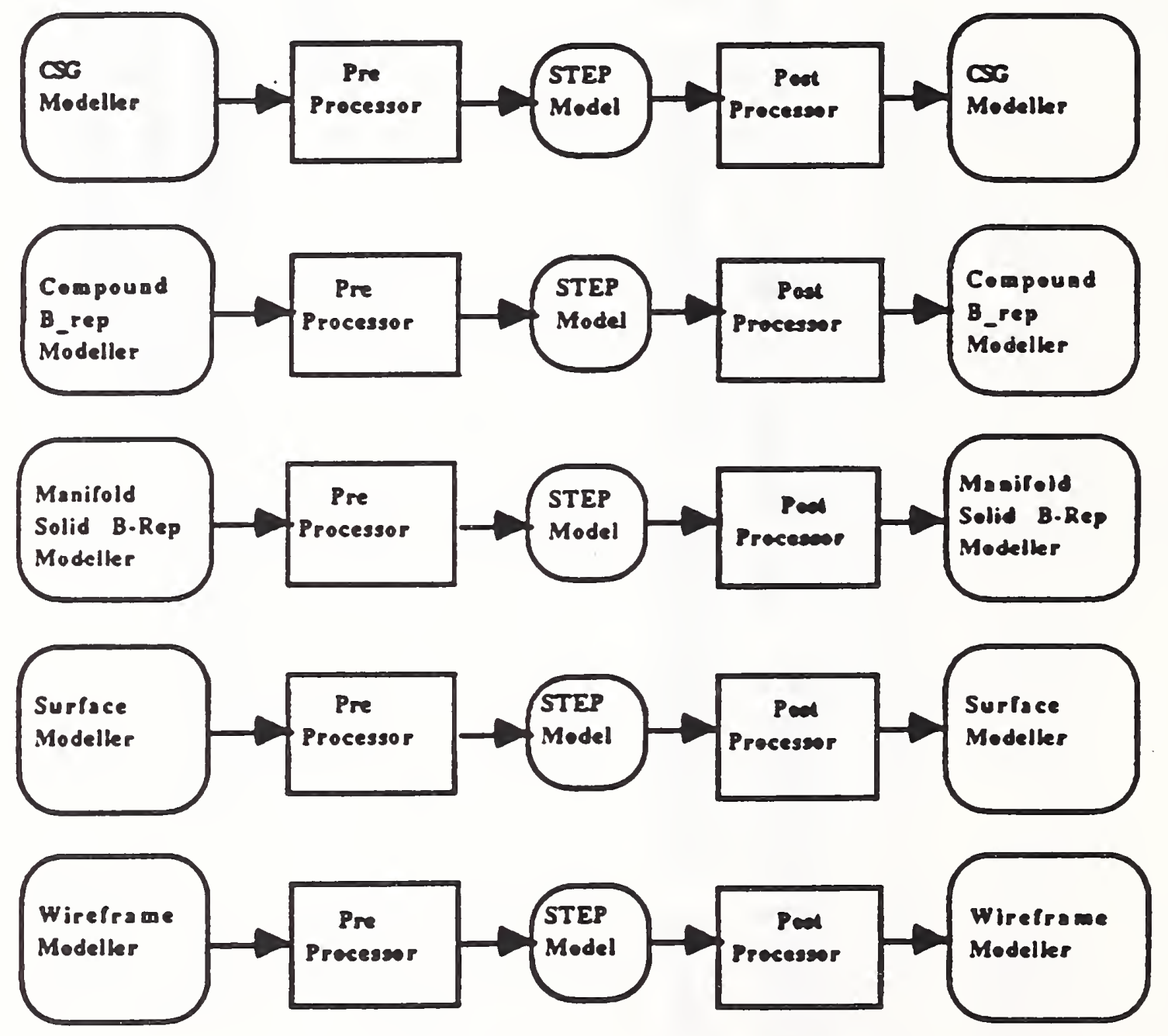


\section{ESPRIT CIM PROJECT 2195}

FAMexchange architecture:

merged processors

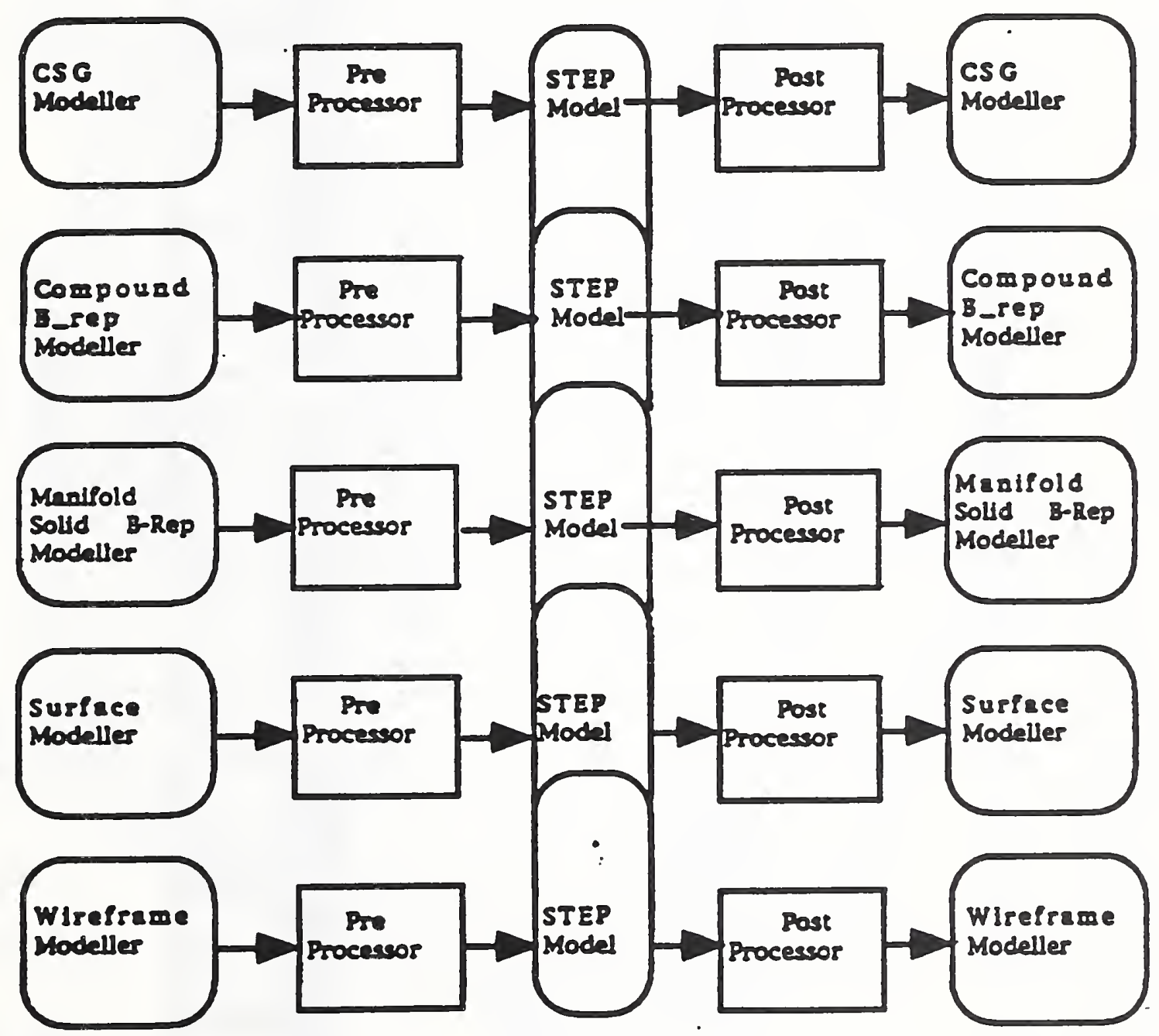




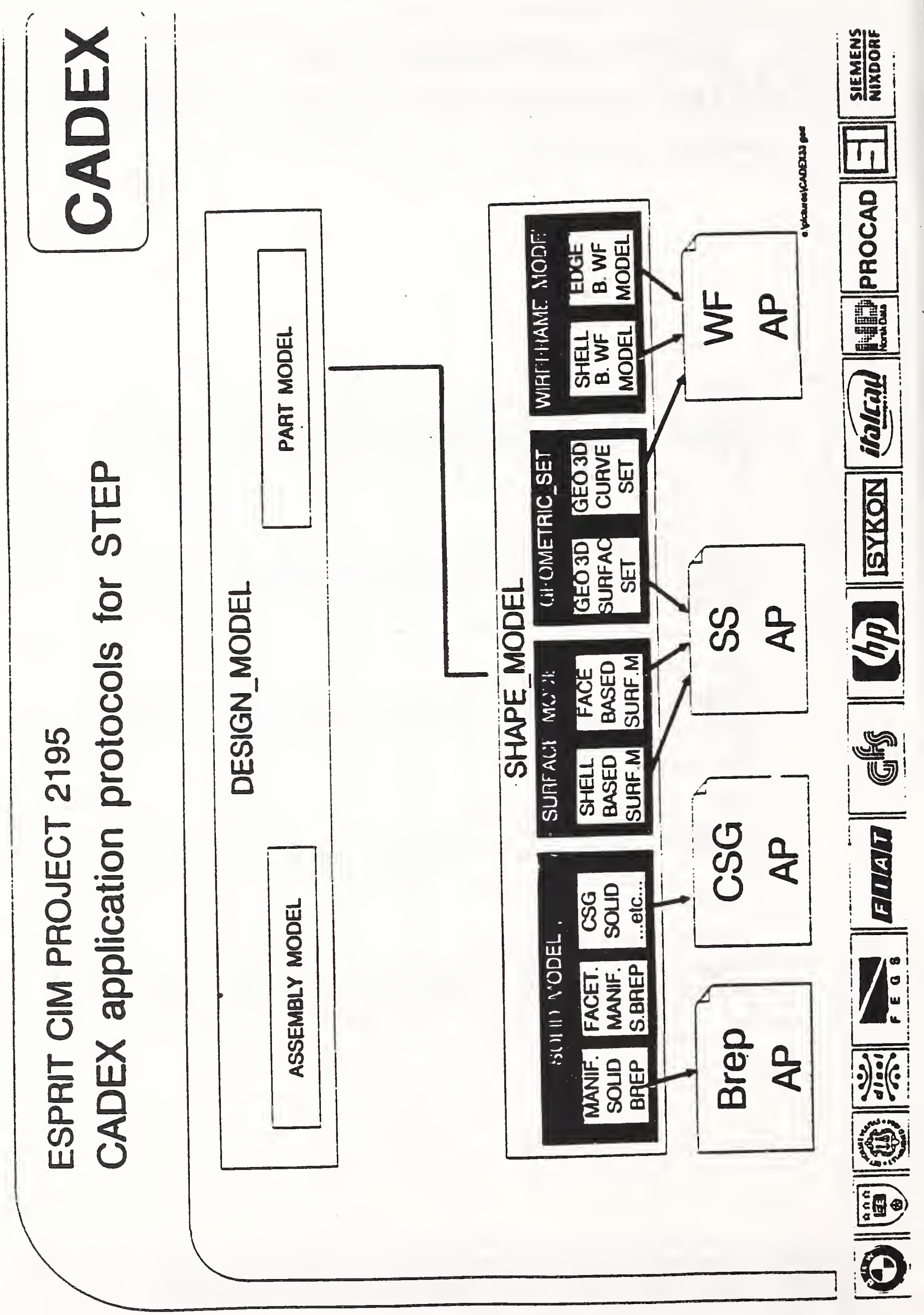




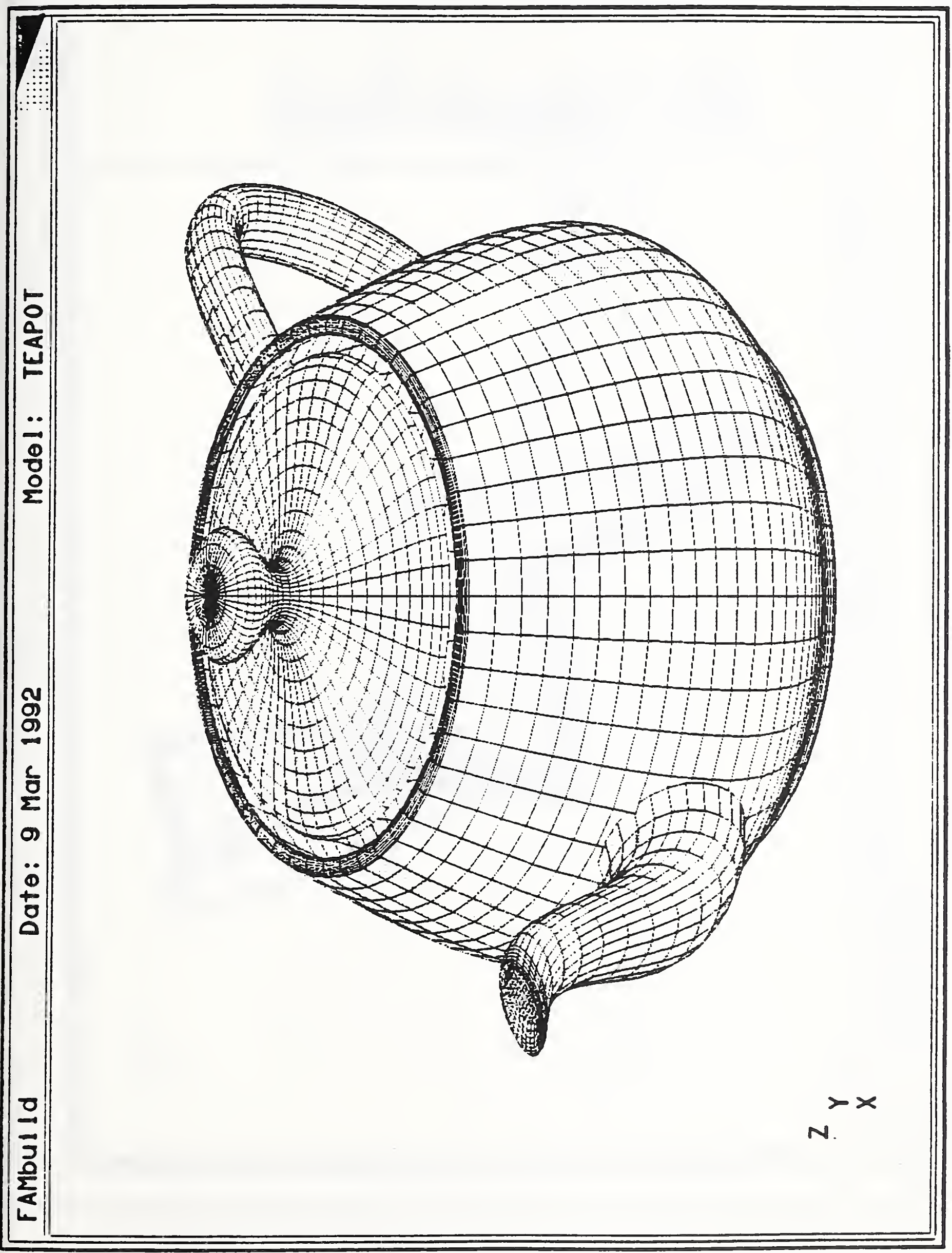




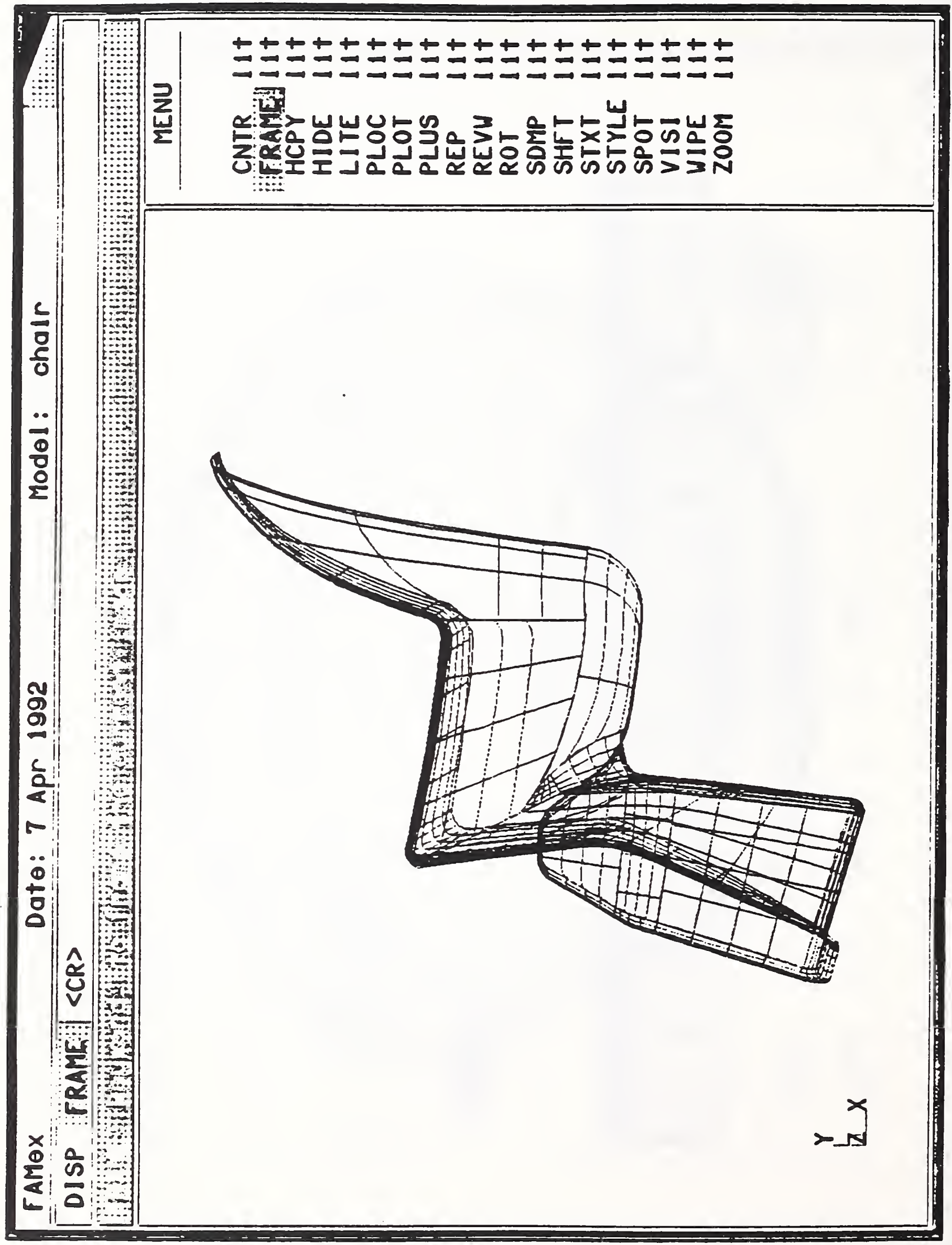




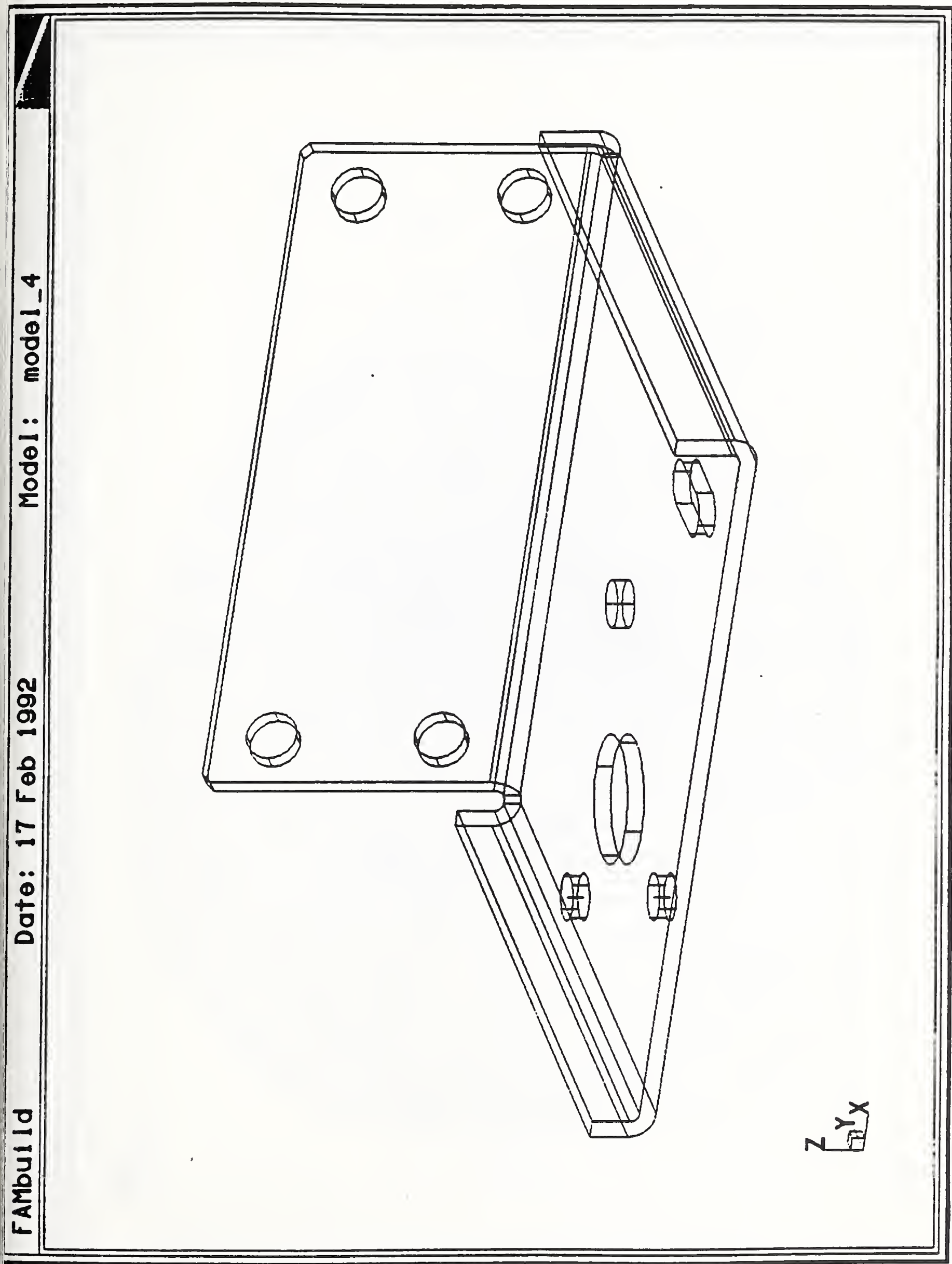




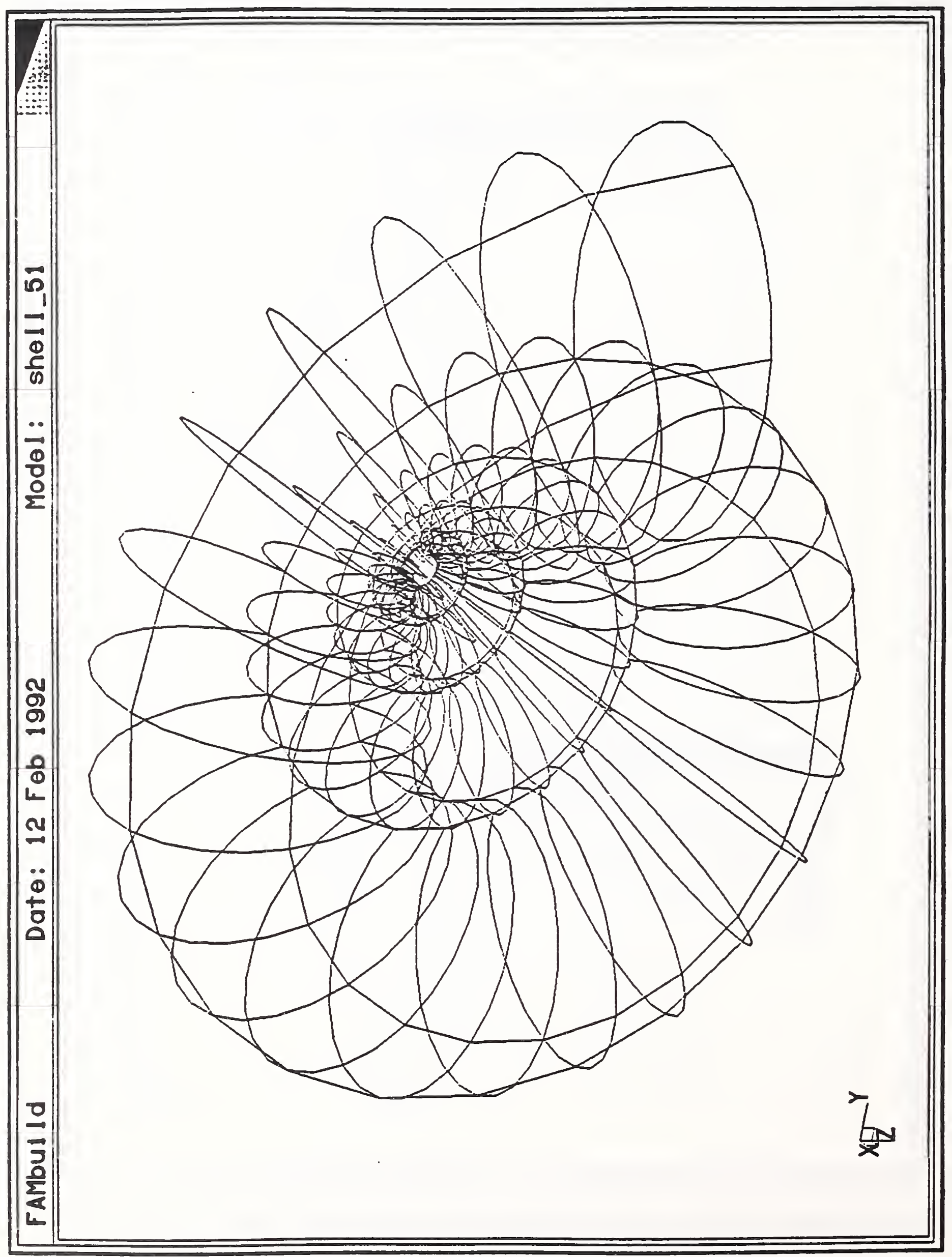




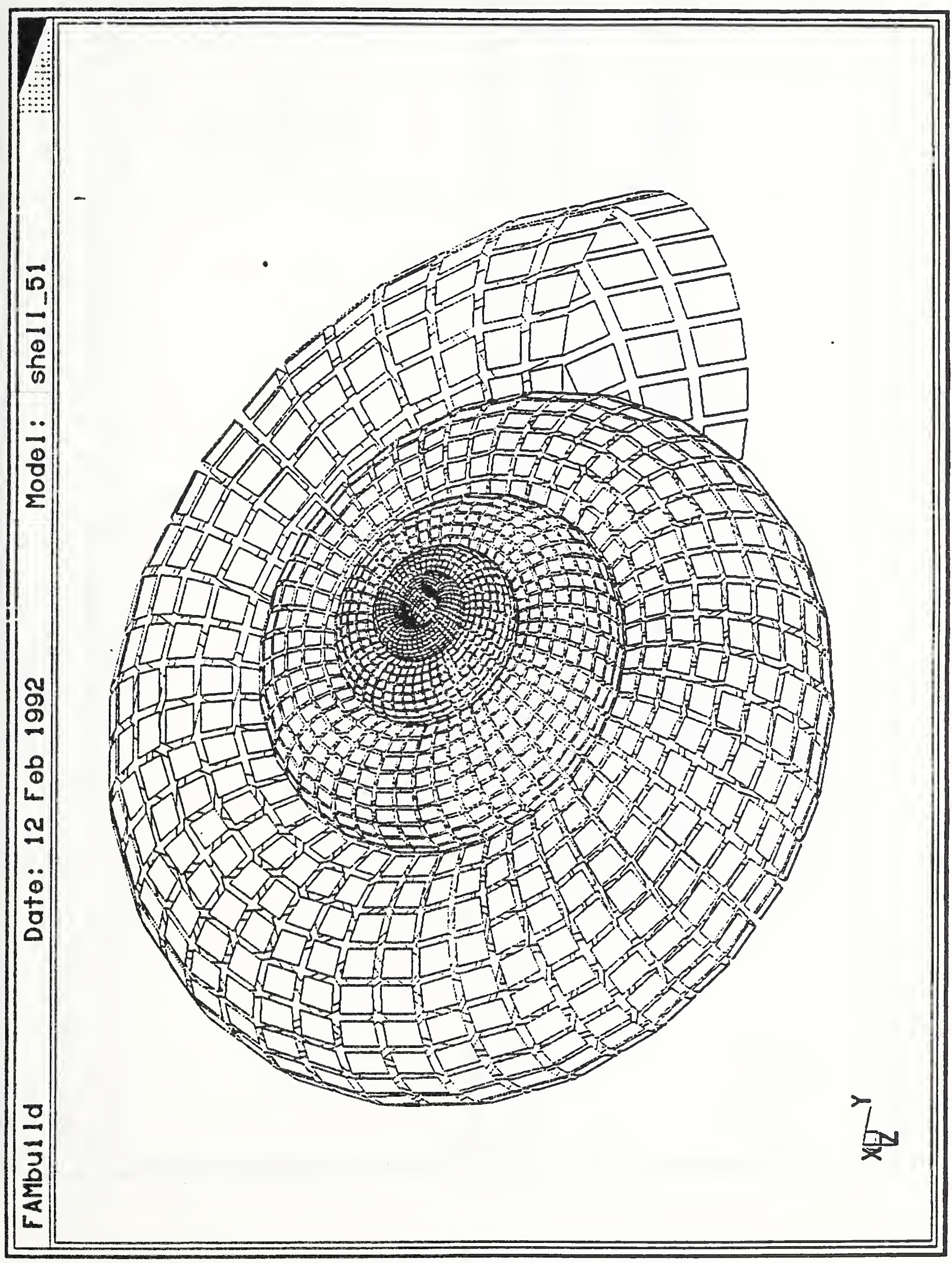



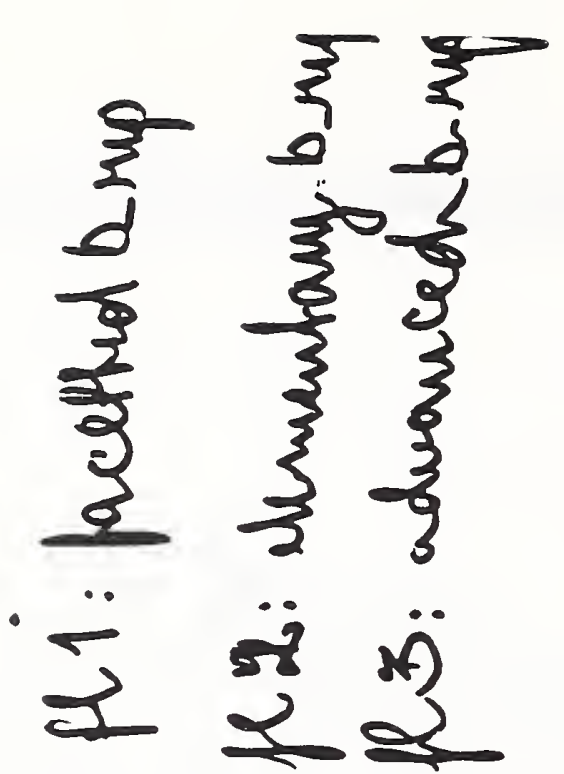

袁喜索

N.
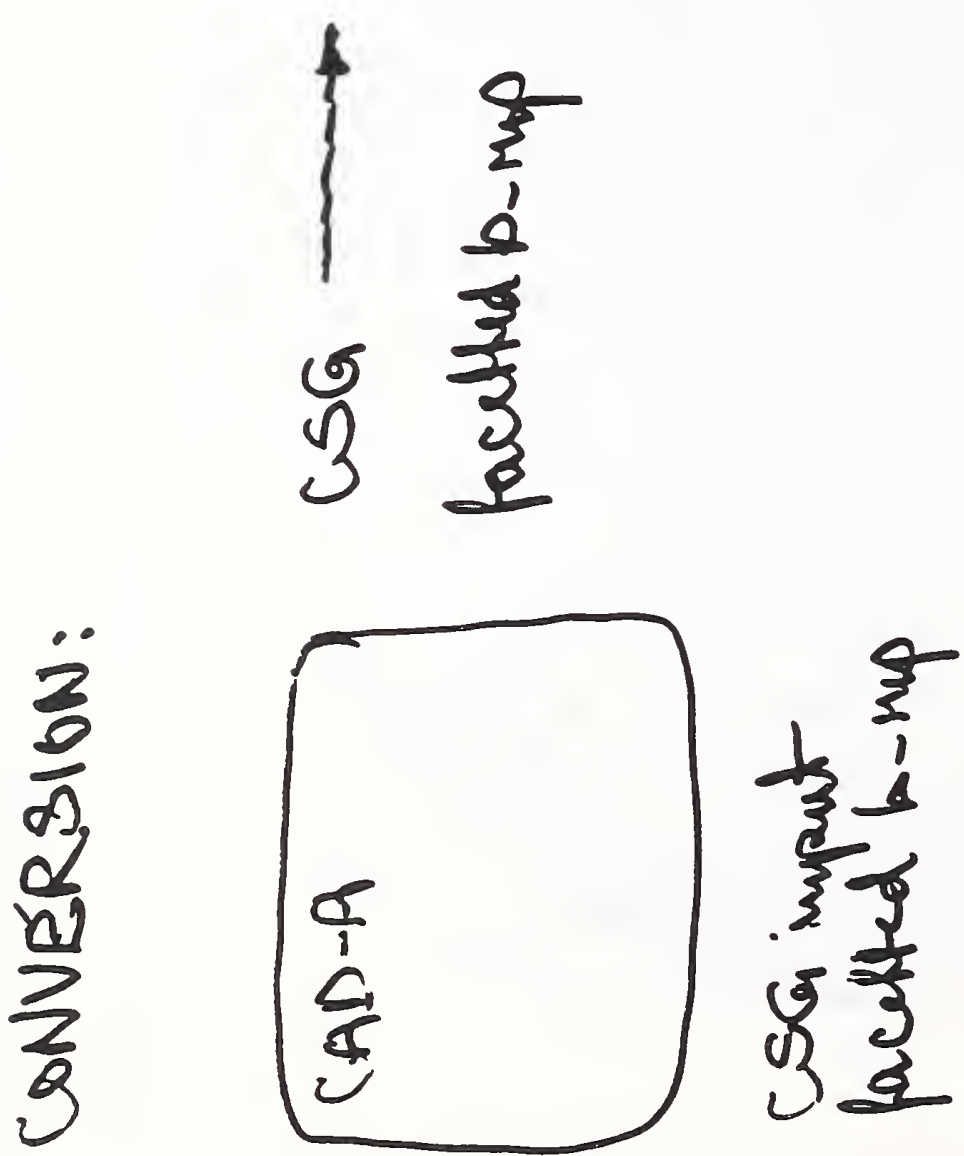


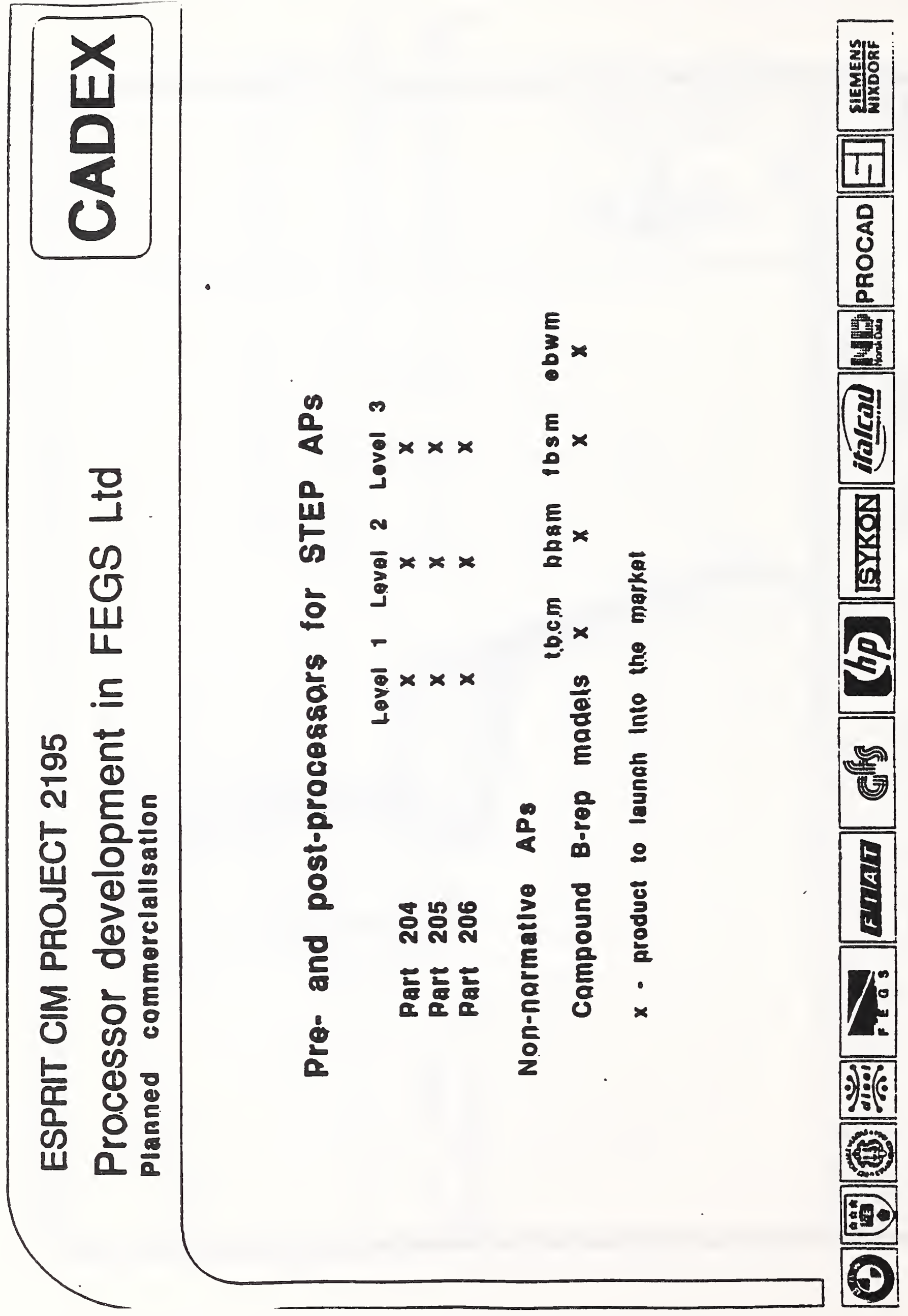




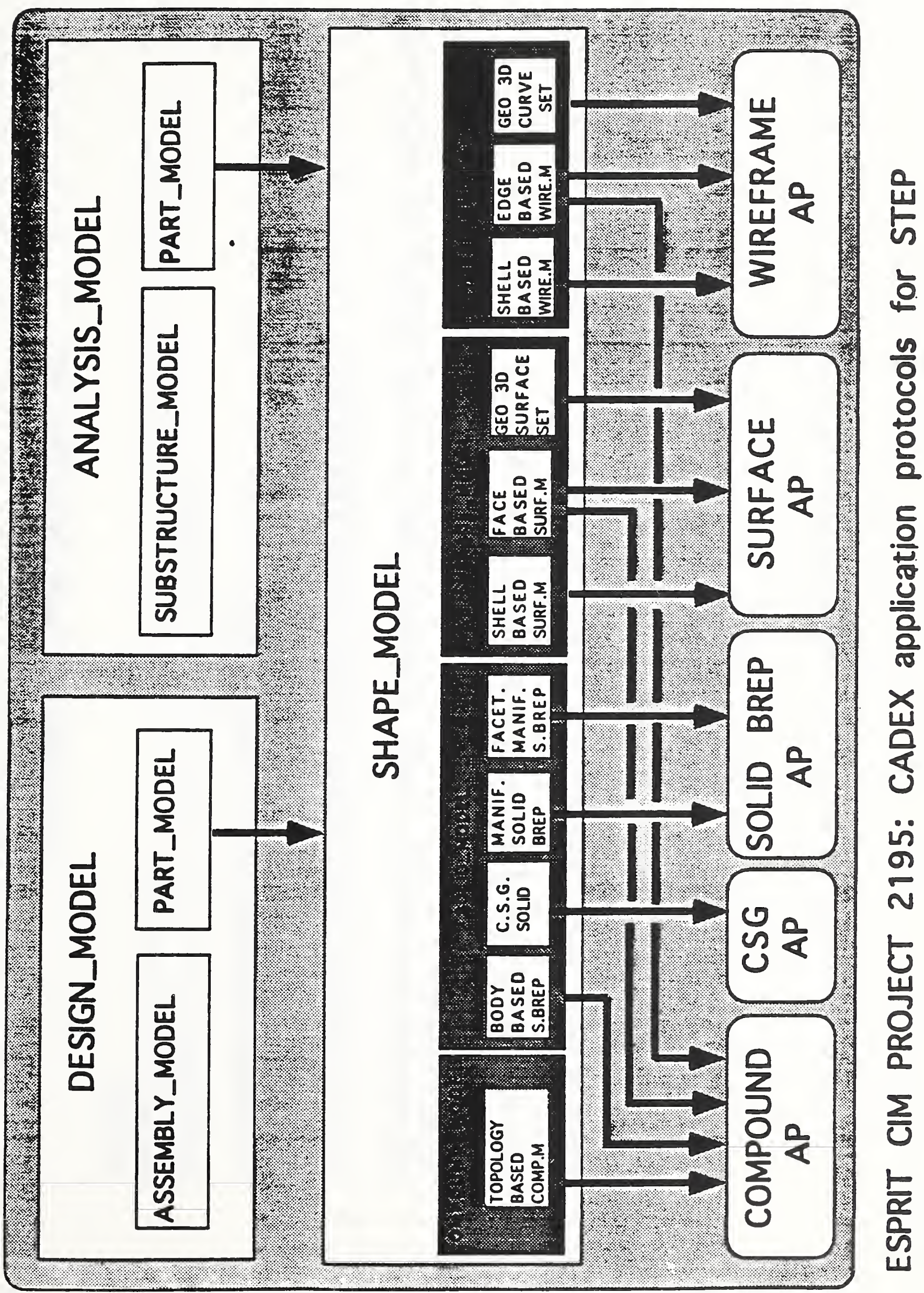




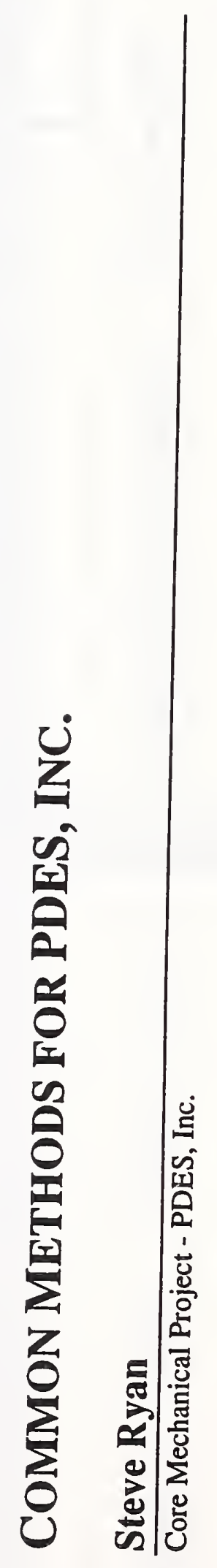




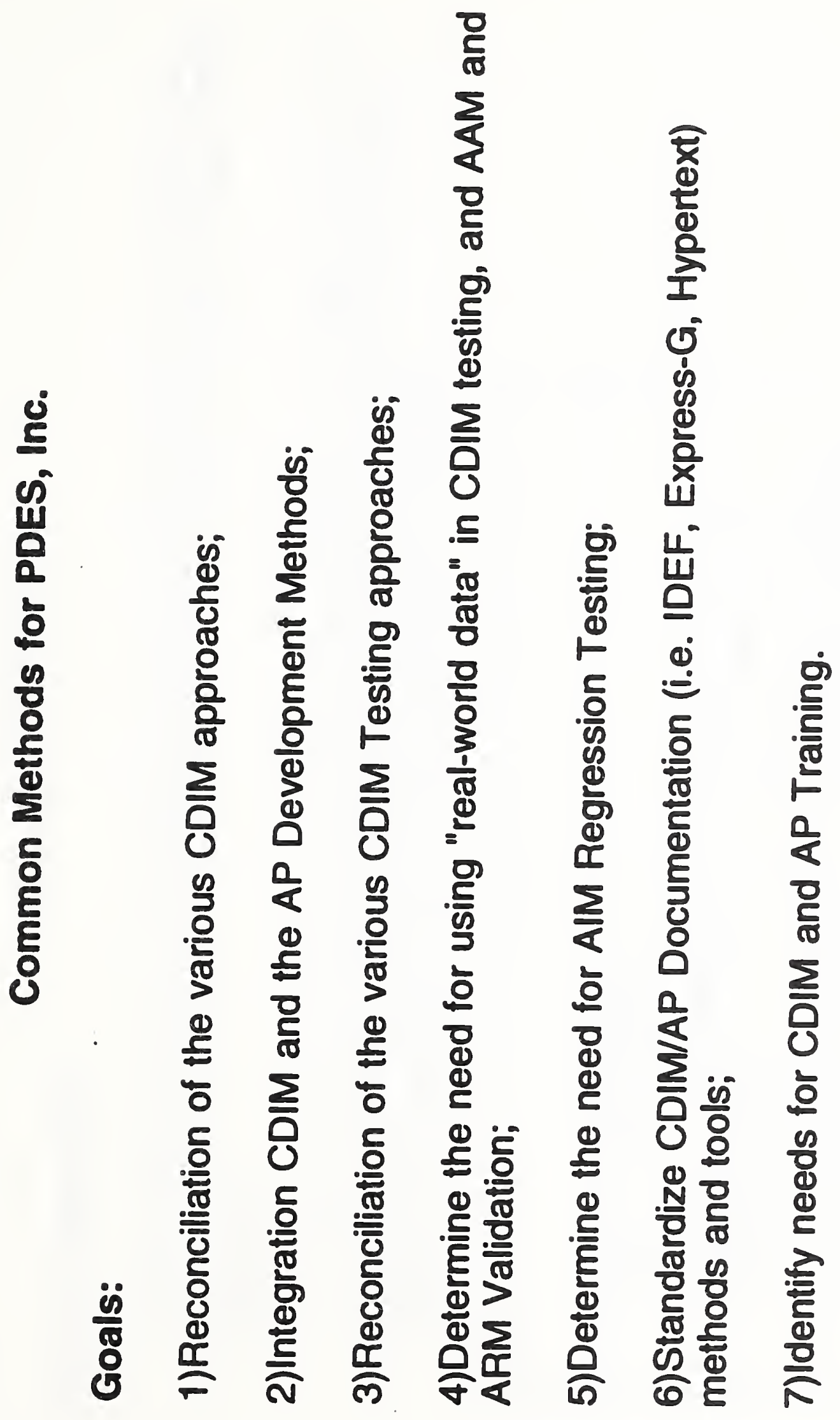




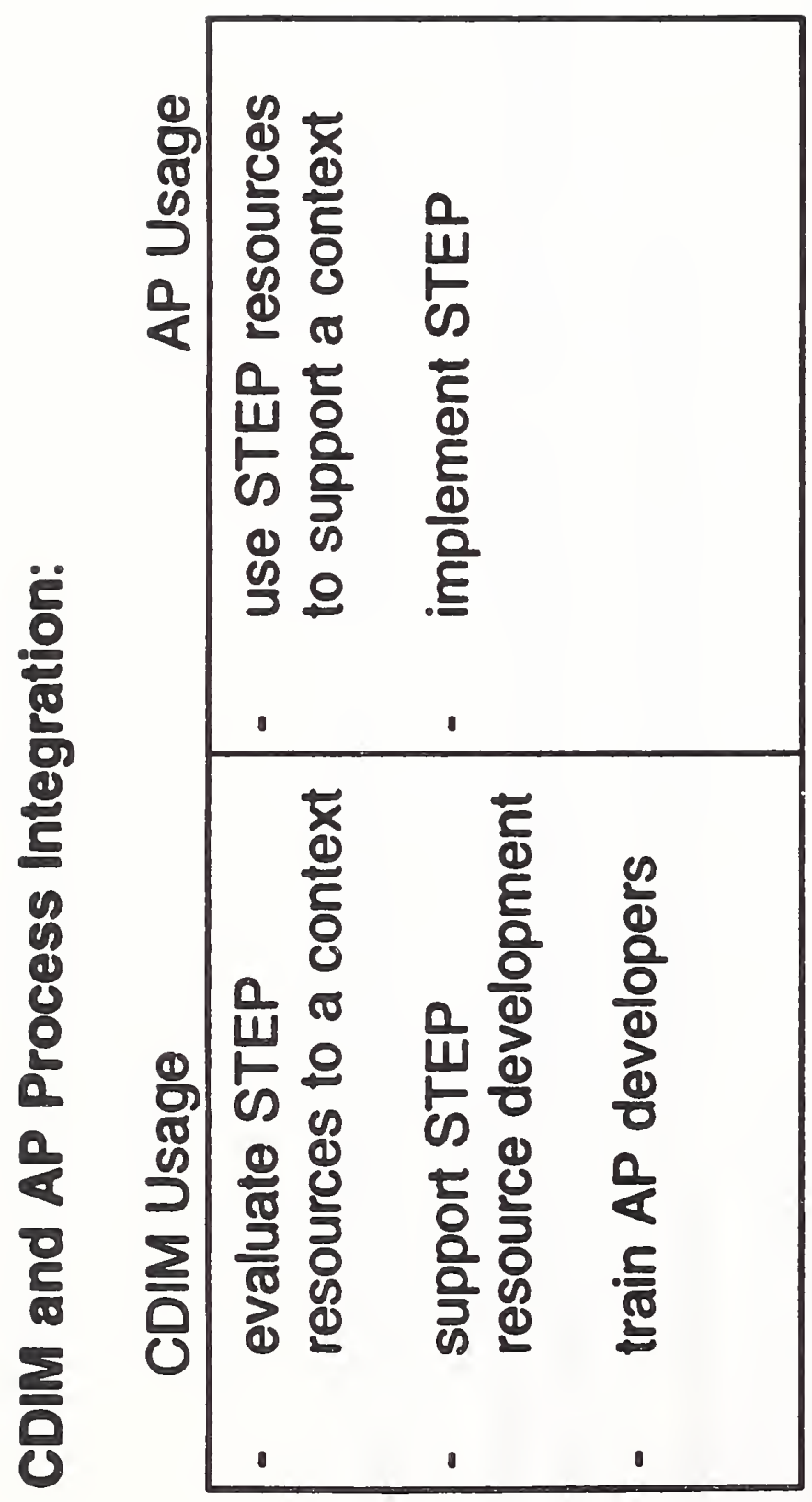




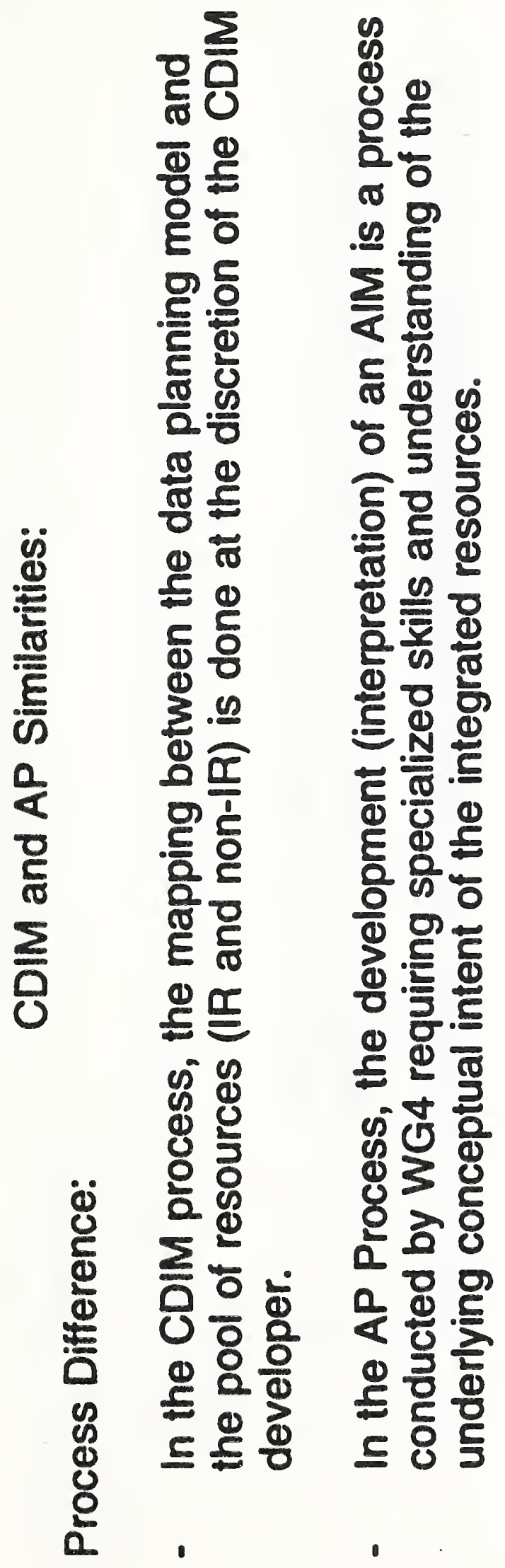




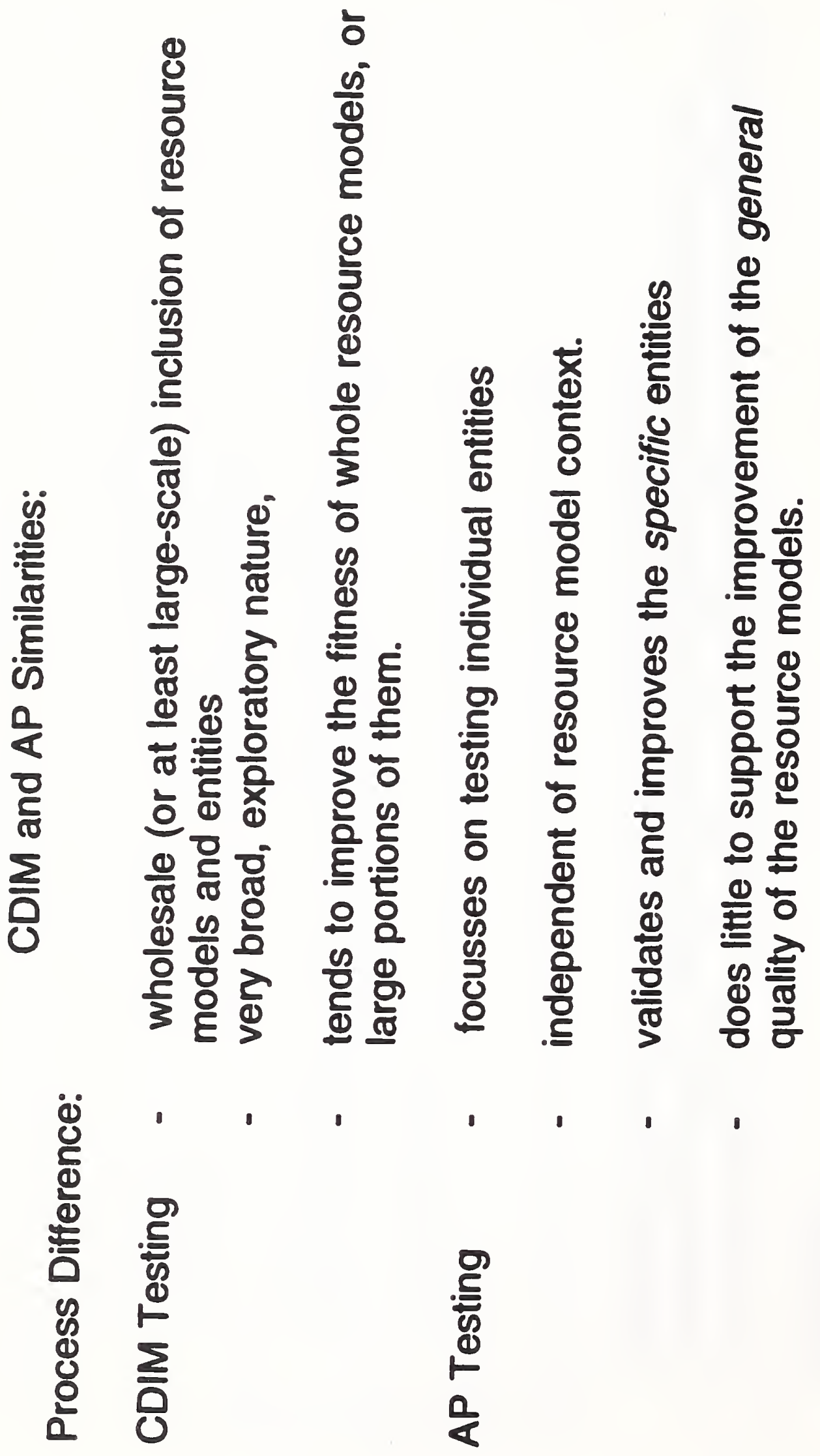




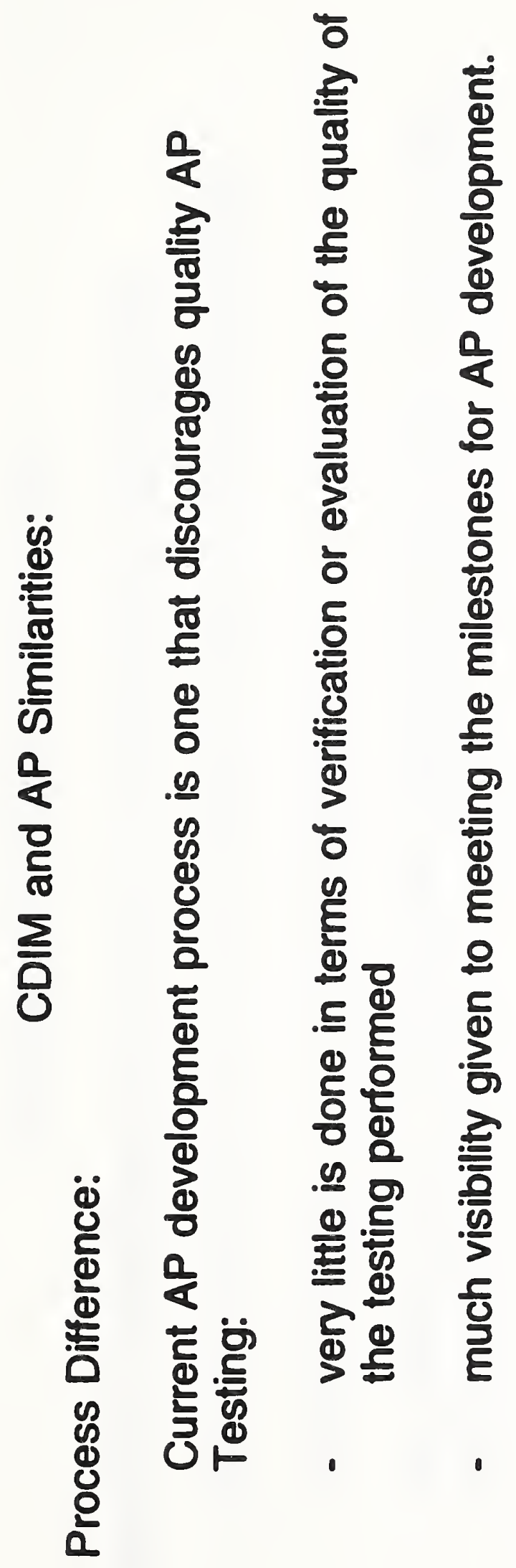




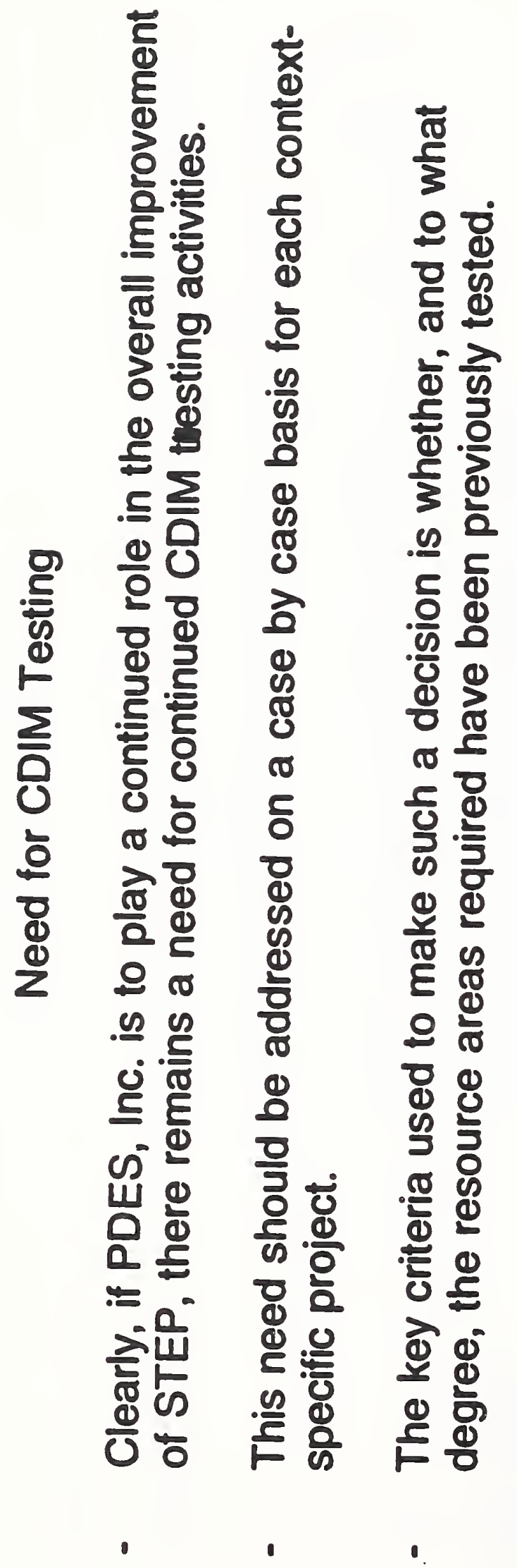




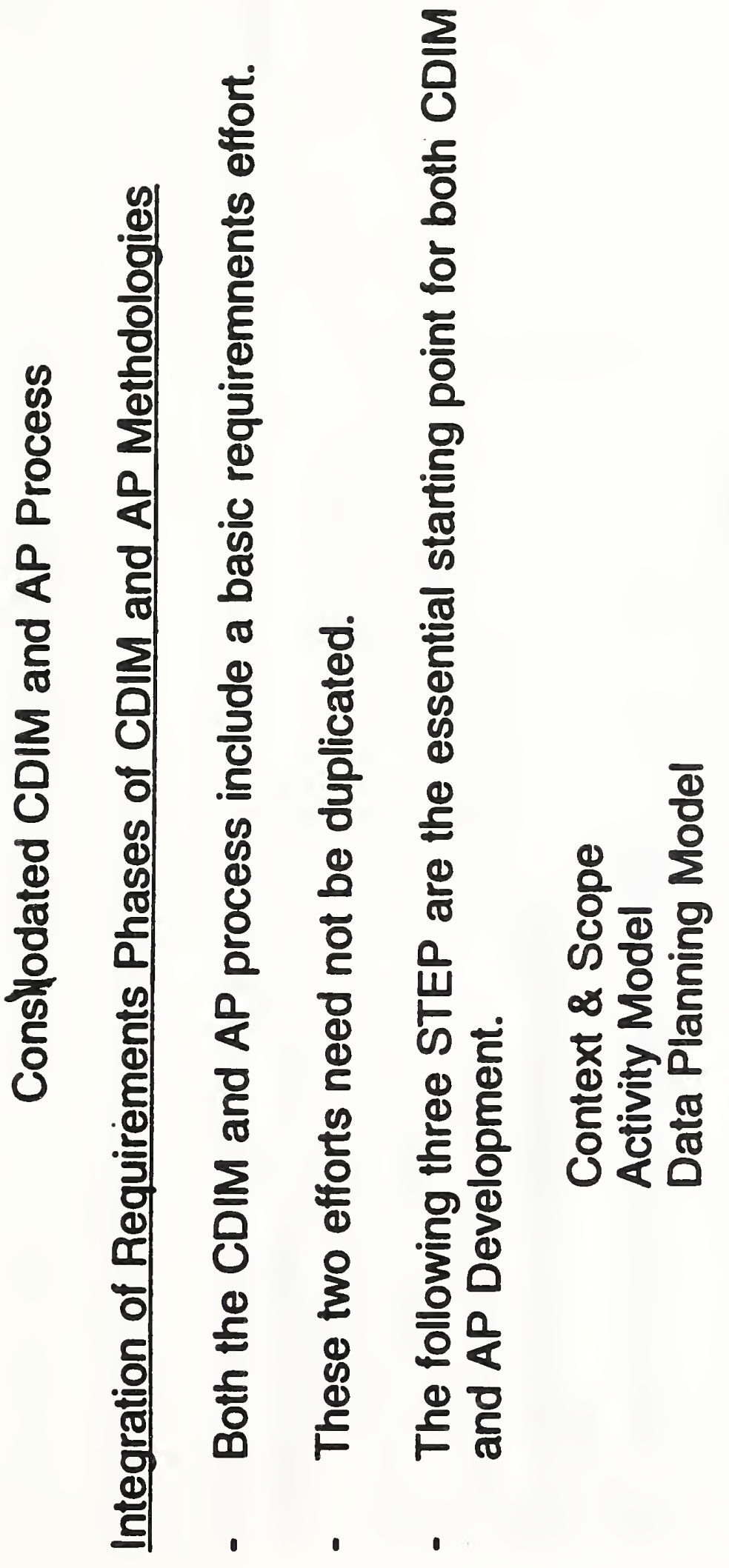




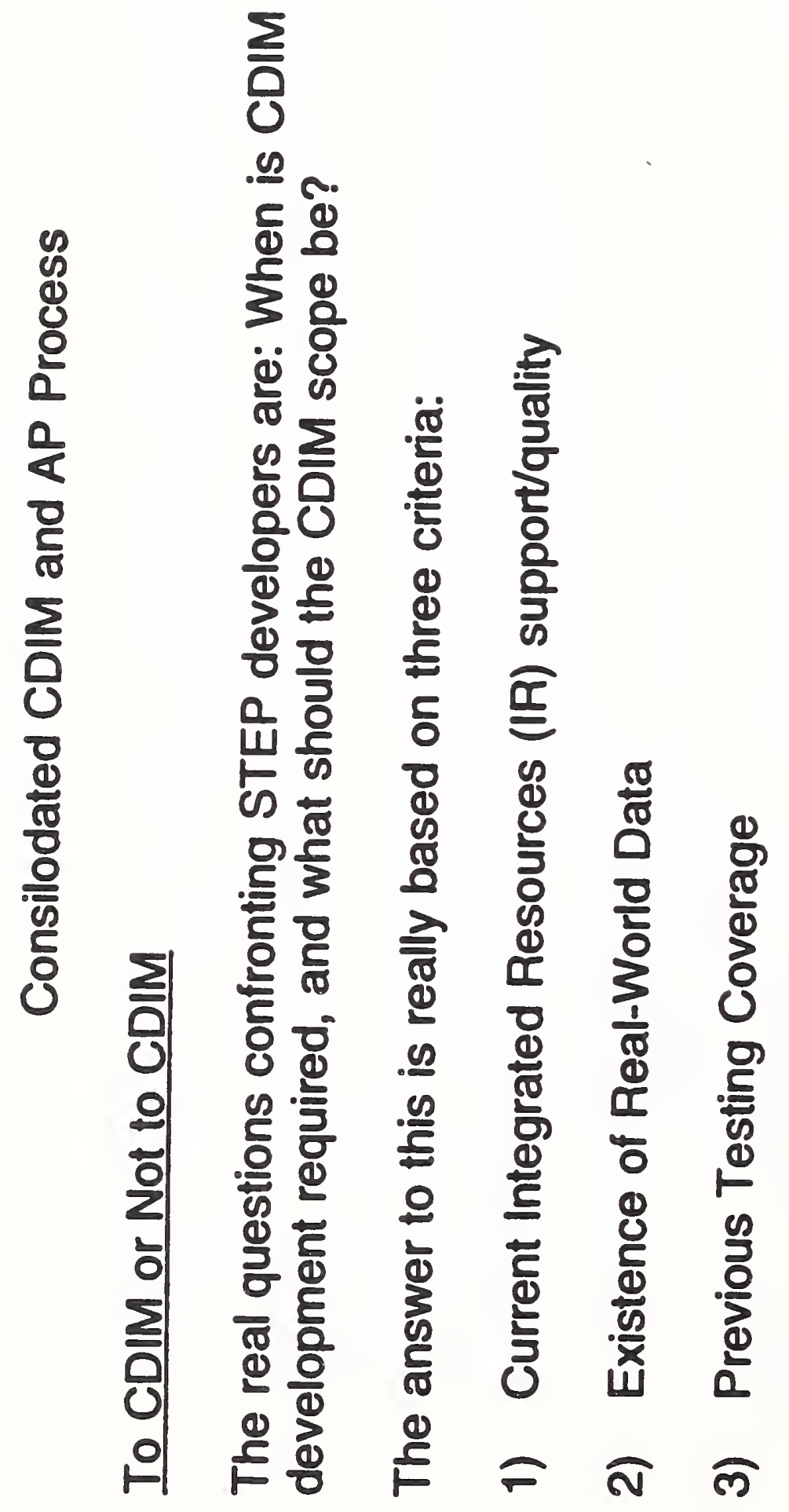



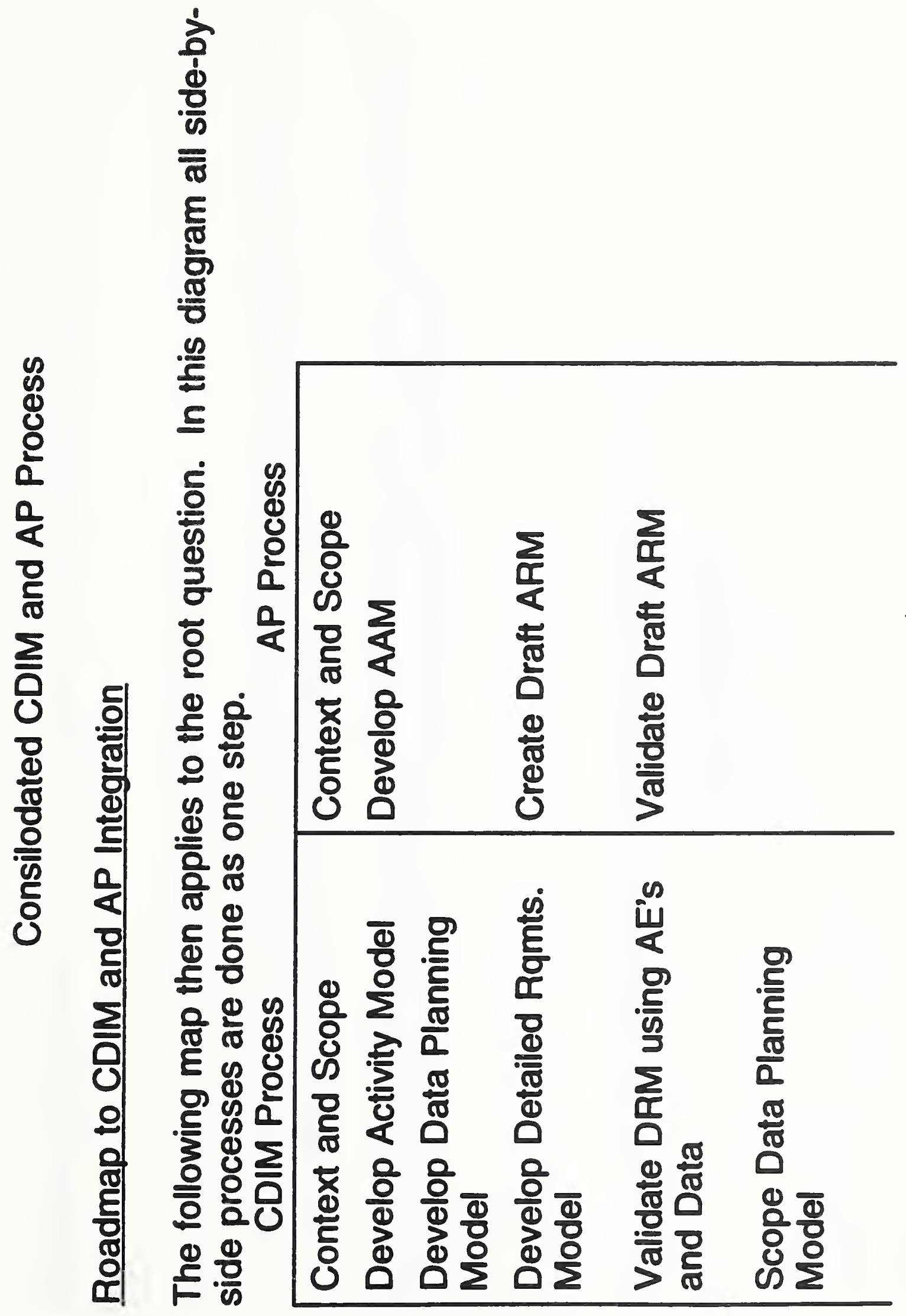


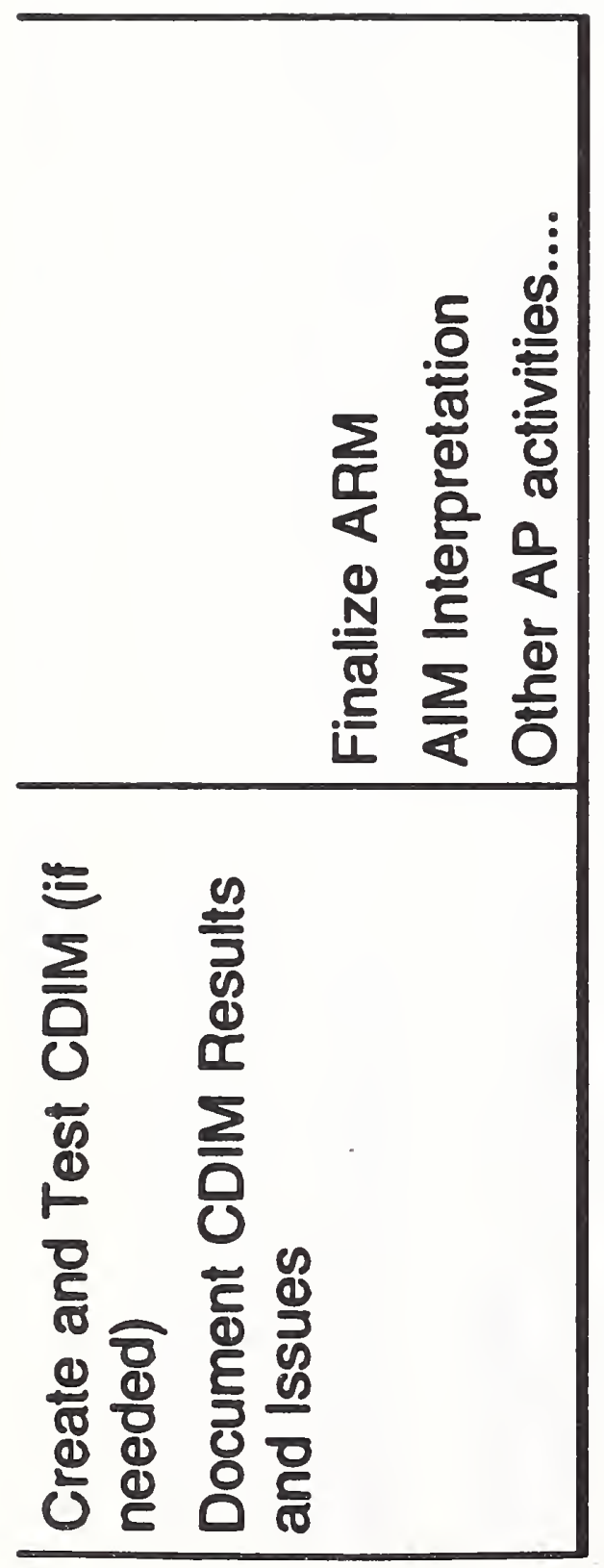




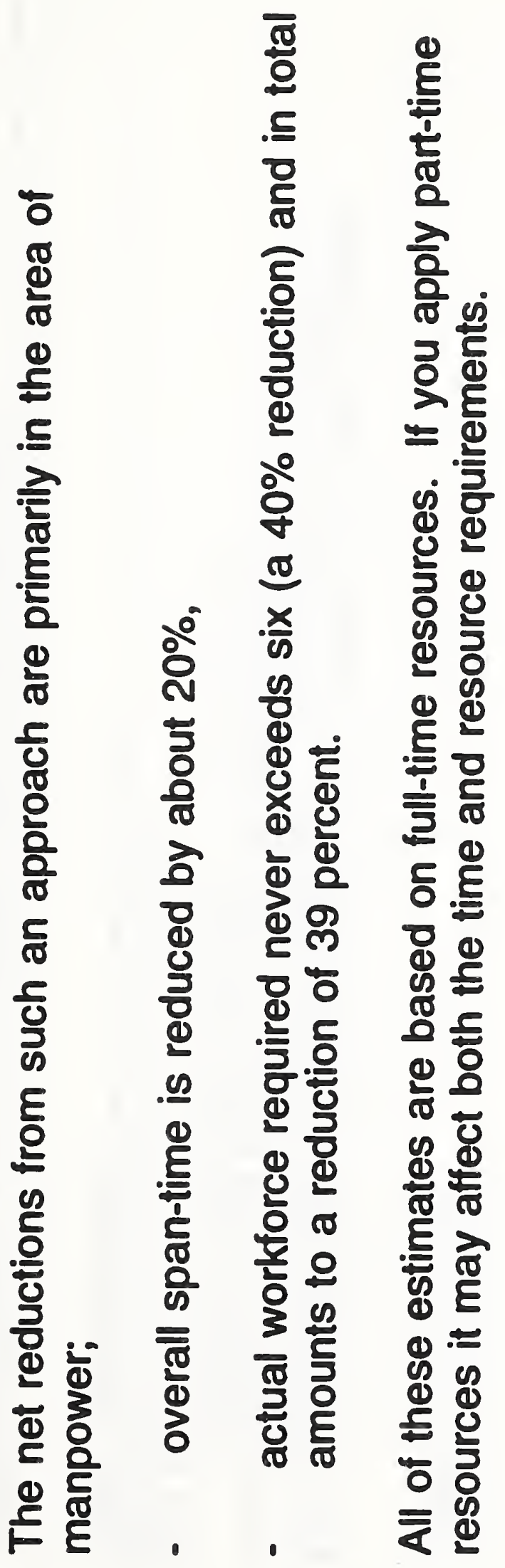




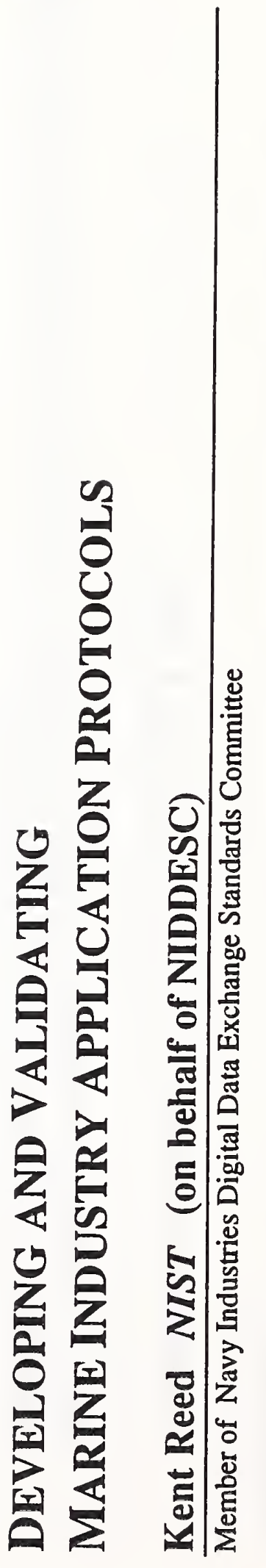




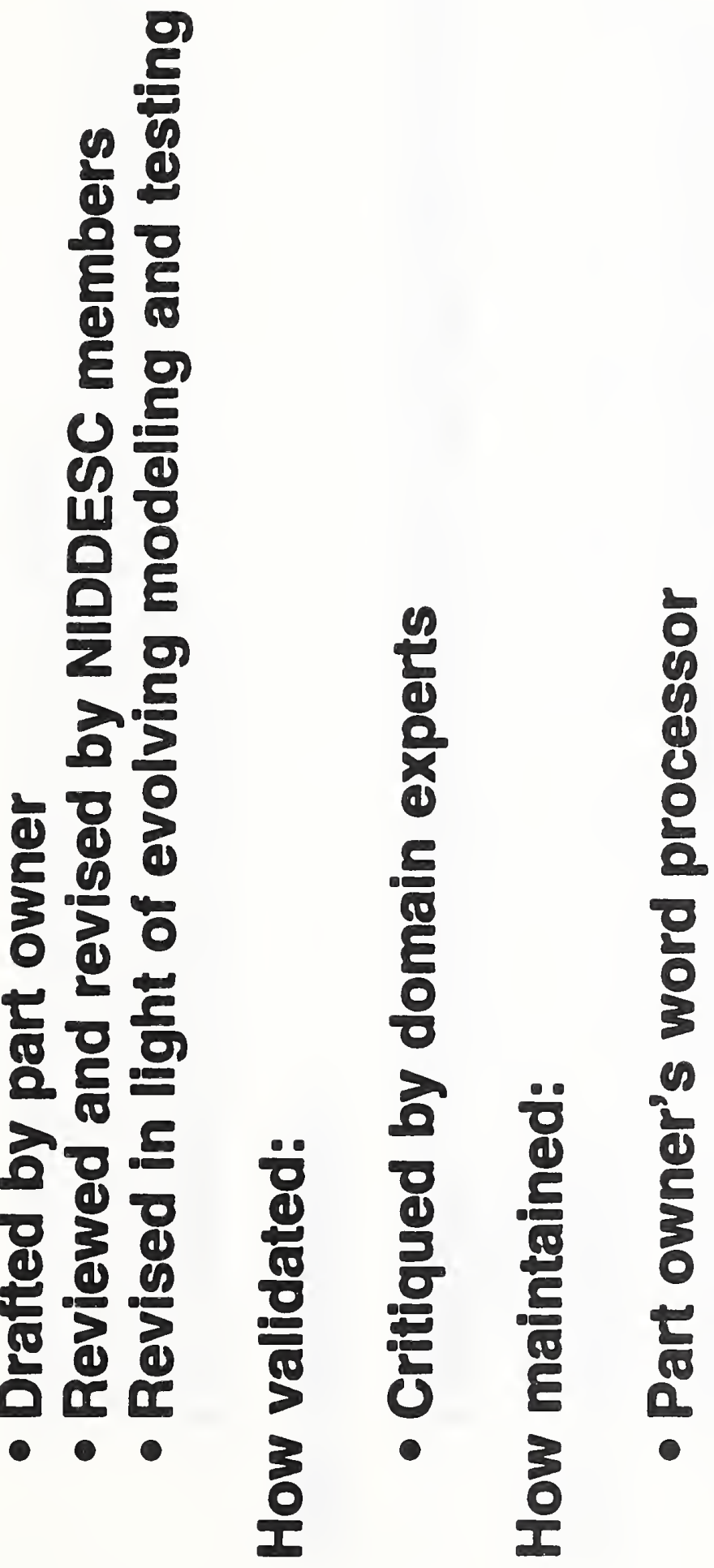




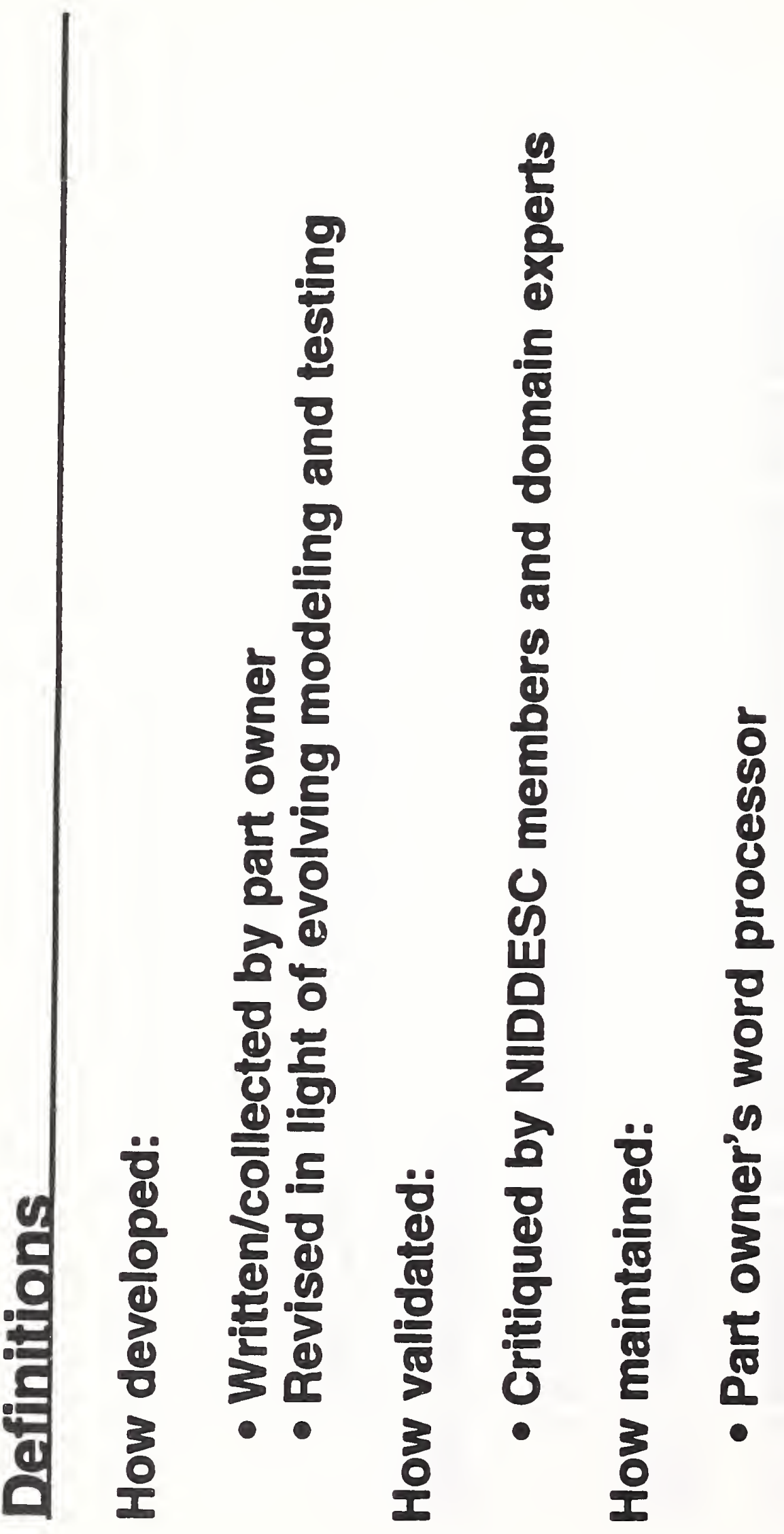




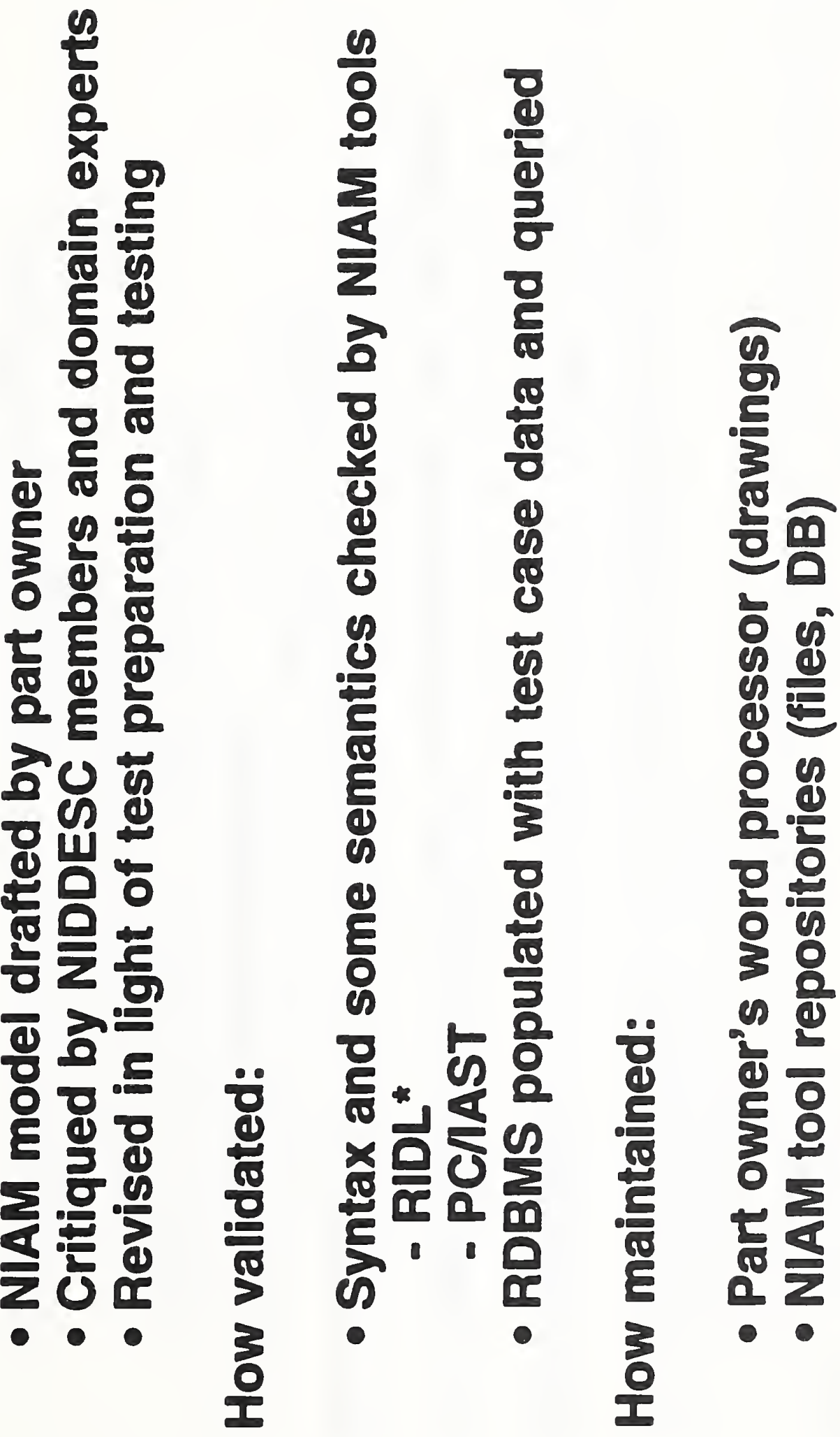




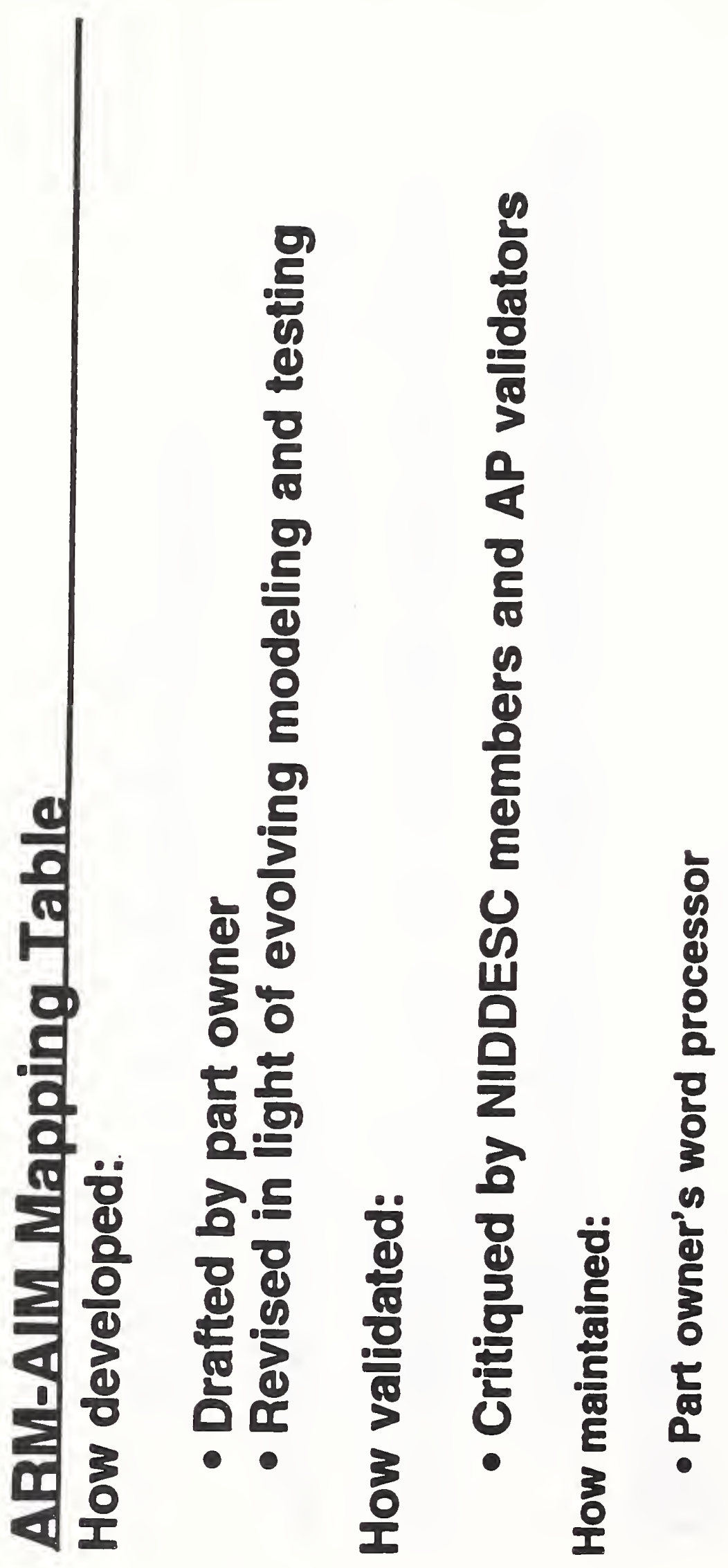




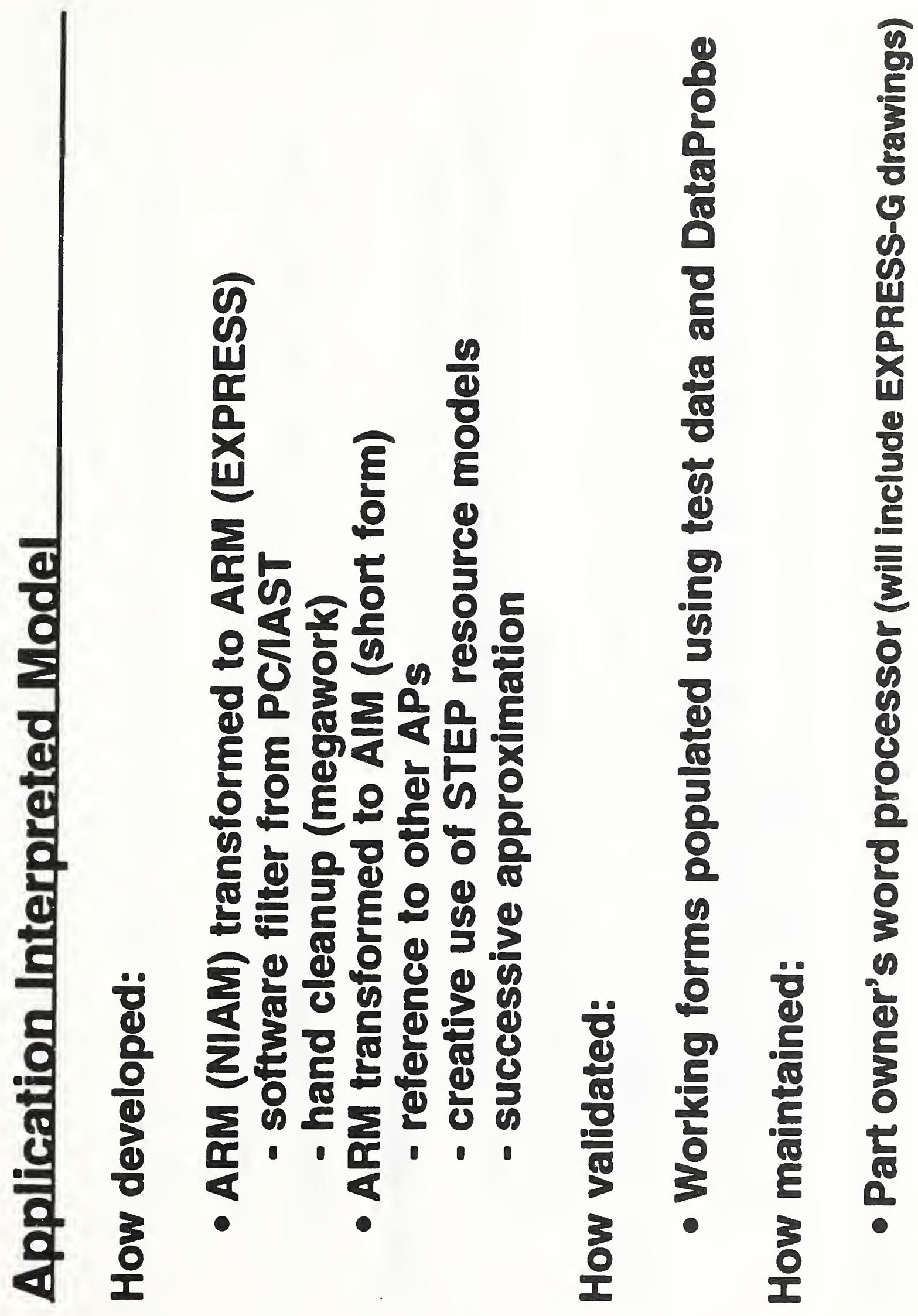




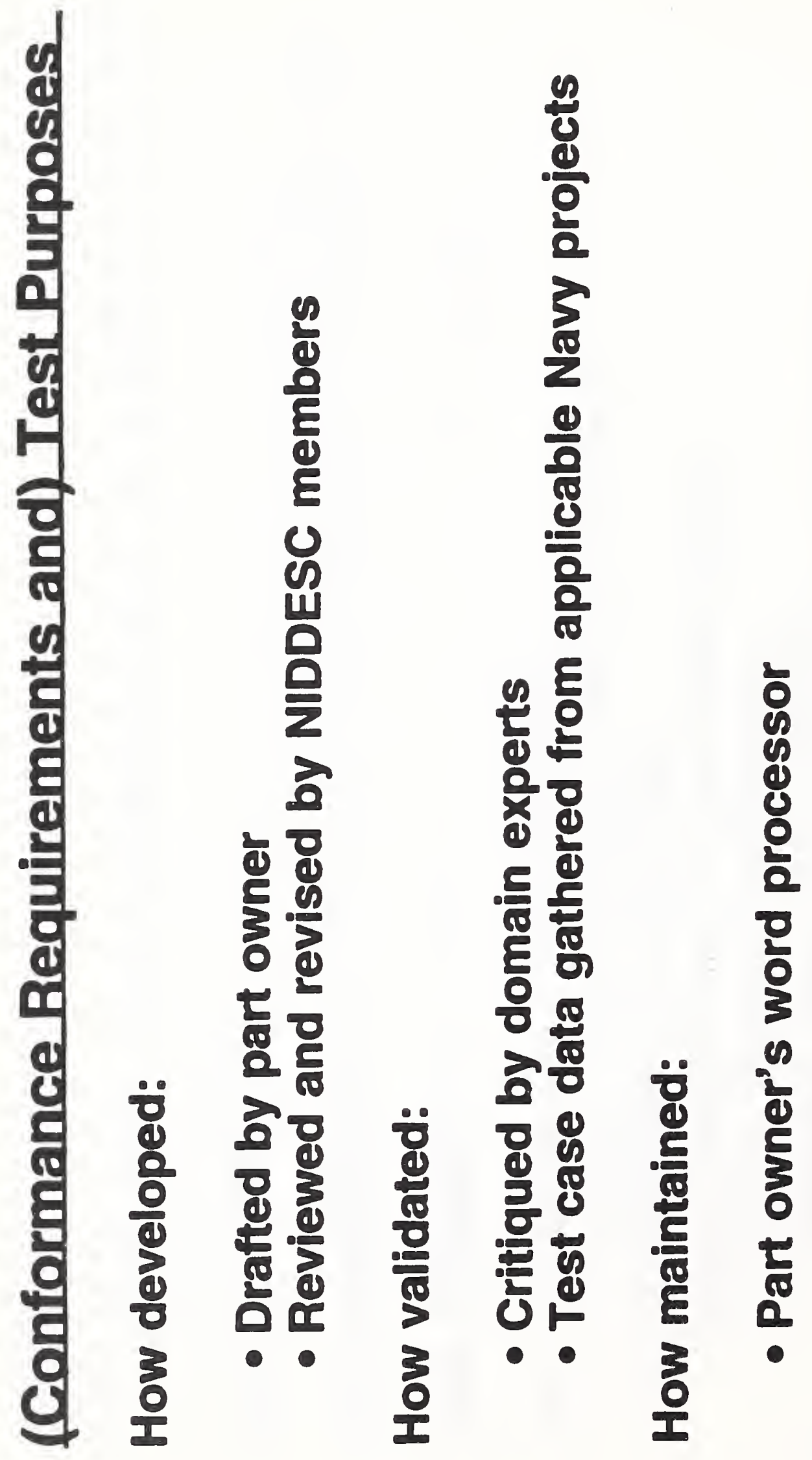




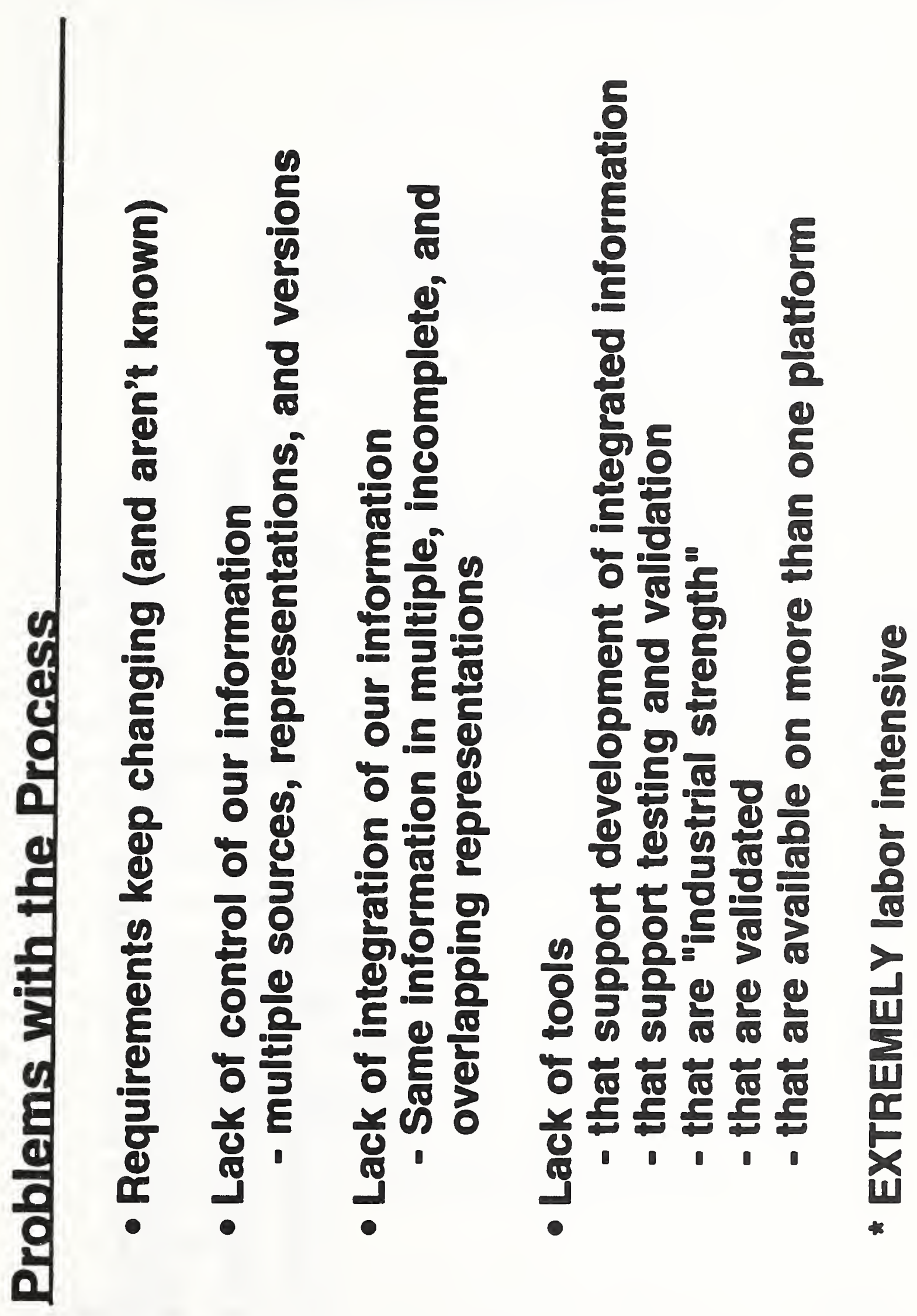




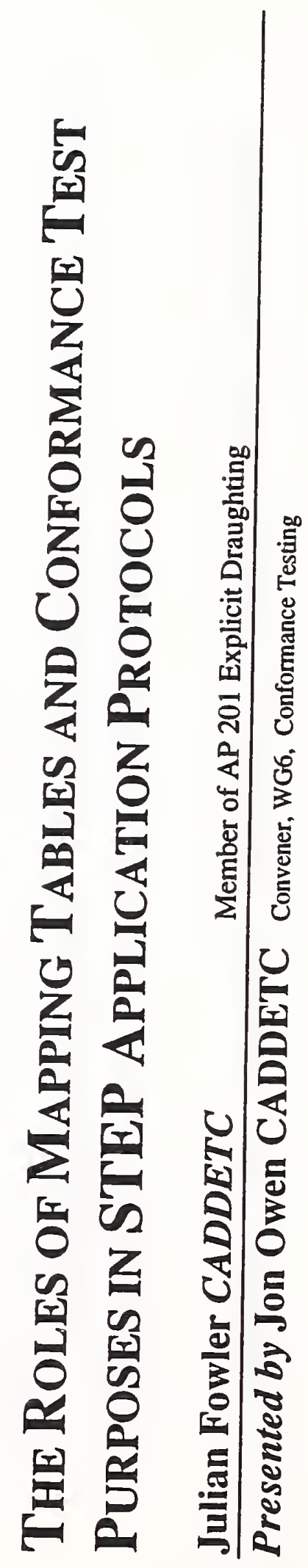




\section{The Roles of Mapping Tables and Conformance Test Purposes in STEP Application Protocols}

\section{Agenda}

- Background

- What is an AP?

- Relationships between components of an AP

- The pole of the mapping table

- Contents of the mapping table

- current position

- future requirements

- Conformance test purposes

- what they are

- how they are developed

- how they are documented

- Relationship between mapping table and conformance test purposes

- Usage test purposes 
- Mapping tables and test purposes have been required components of STEP Application Protocols since the AP was adopted as part of STEP in 1989

- Active work on APs has to date concentrated on AAMs, ARMs (the AP developers), AIM development and integration

- Approaches to mapping tables and test purposes have not been agreed or documented: note the differences between Parts 201 and 203

- The key role of the mapping table has emerged in consideration of how test purposes relate to the AP and to the abstract test suite

- This presentation summarises the current position and proposes how we should move forward

\section{What is a STEP Application Protocol?}

- A STEP AP is a Standard

- A STEP AP is a specification of data constructs (entities and structure) required to exchange and/or share product information to meet a documented industrial requirement

- A STEP AP may specify several "levels": each of these could be documented as a separate AP Part

- A STEP implementation combines a STEP AP and an implementation form (physical file, SDAl, etc)

- A STEP AP is not a subset of the STEP Integrated Resources

- STEP APs are to be integrated to enable interoperability between implementations of different APs that share common information

- "Application Interpreted Constructs" specify potentially shareable information structures 


\section{STEP AP Interoperability}
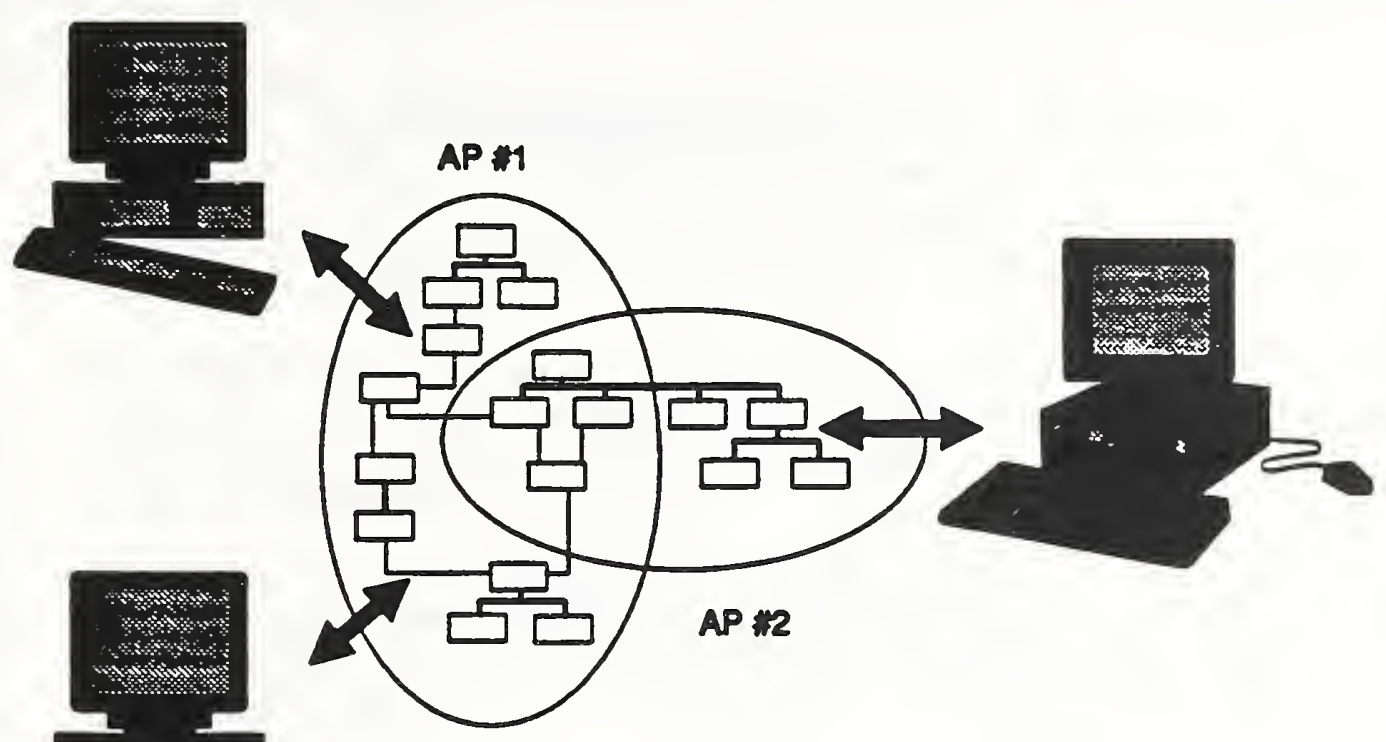

2

s.

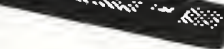

Relationship between AP components

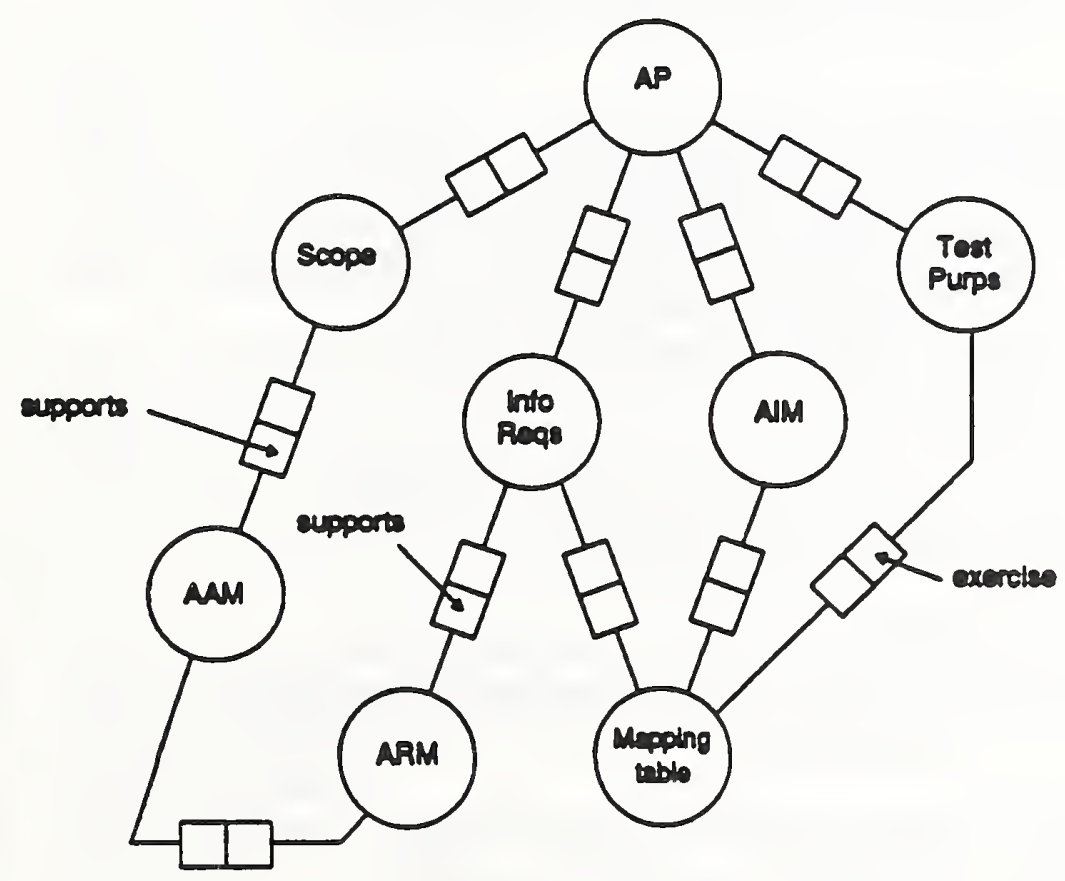




\section{AlCs as complete, testable "mini APs"}

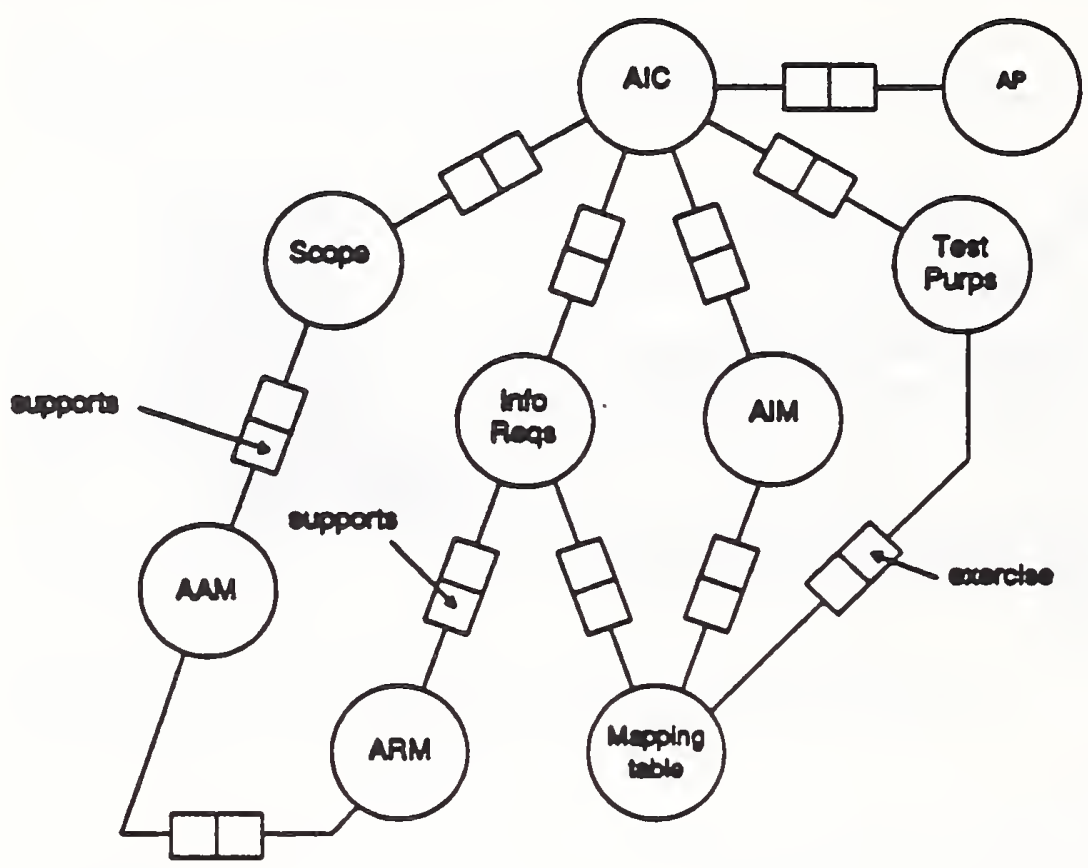

\section{The role of the mapping table}

- The mapping table documents the correspondence between the information requirements and the AlM. The listing shall provide a complete and unambiguous mapping between the constructs defined in clause 4 and the constructs defined in the AlM, including preservation of the construct assertions."

(Guidelines for the development and approval of STEP Application Protocols, version 1.0, WG4 N34)

- The mapping table provides an explicit, normative link between the application domain and its language, and the "STEP" domain and its language.

- The level of detail and length of the mapping table depends directly upon the level of detail to which the information requirements are modelled. 


\section{Contents of the mapping table}

- In developing Part 201 a two-level mapping table has been employed. The content and layout employed has not yet been agreed from either the technical or editorial vlewpolnt.

- Each ARM construct (entity) is mapped to a "primary" construct in the AIM.

- This level may be sufficient for APs where the mapping is relatively simple, $\theta .9$. the mappings from the geometric requirements in Parts 204, 205 and 206 to the goometry_schema In Part 42

- Where this "primary" mapping is not complete and unambiguous, each attribute of the ARM entity is mapped to one or more AlM constructs; In each case a unique "reference path" must be stated.

- Any and all global rules in the AIM that play a role in satistying the ARM requirement are stated explicitly.

\section{Future requirements for mapping tables}

- Current approach results in a mapping table that is only useful for determining a "One way" mapping: ARM $\rightarrow$ AIM; consideration of test purposes suggests that a two-way table (or tables) may be required; i.e., given an AIM construct, which ARM construct(s) does it play a pole in satisfying?

- The mapplng table must satisty the need: "what query or queries on an Implementation of the AIM will give all the data necessary to populate a CAx system database instance corresponding to the given ARM construct?"

- Mapping tables should be computer-processable.

- Is the above requirement synonymous with, and/or dependent on, a need for:

- standardised, formal method(s) for documenting requirements;

- a standardised mapping language use in the interpretation process? 


\section{Conformance test purposes}

- A conformance test purpose is ... "a precise description of the objective which an abstract test case is then designed to achieve."

(iSO CD 10303-31 "Conformance Testing Methodoiogy and Framework")

- A conformance test purpose, taken together with the aspect of the mapplng table exercised by the test purpose and the corresponding parts of the information requirements and AIM, is a specification of an abstract test case.

- Conformance test purposes identify ail the discrete options in the AIM: OPTIONAL attributes, subtypes, select types, enumerations (including BOOLEAN and LOGICAL)
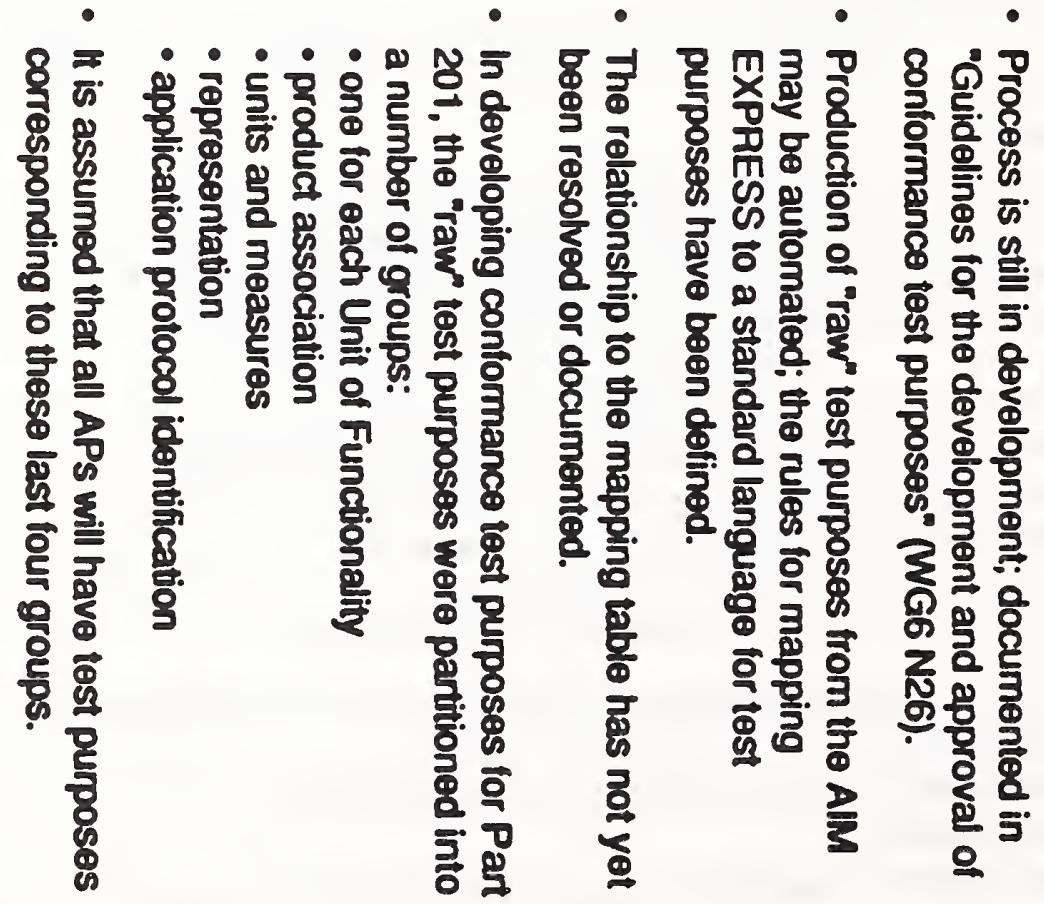


\section{Documentation of conformance test purposes}

- Test purpose name is that of the corresponding AlM entity.

- The context(s) In which the test purpose exists are listed; these are all the roles that the AlM entity plays.

- All discrete options In the entity are listed, using a standard languago.

- TEST_PURPOSE = ENTITY_NAME ("AS" ENTITY_TYPE) I "WITH" ATTRIBUTE_NAME ATTRIBUTE_OPTIONS ).

- ATTRIBUTE_OPTIONS = "NOT PRESENT" I

[ "PRESENT"]

["HAVING AT LEAST ONE ELEMENT" ["PRESENT"]]

("AS" ENTITY_TYPE I"=" ENUMERATION_VALUE").

\section{Relationship between mapping table \& test purposes}

- We know "intuitively" that there is a close relationship between the mapping table and the test purposes.

- An algorithm exists for the generation of test purpose "syntax" from the AIM EXPRESS schema (this has not, as yet, included consideration of the effect of local and global rules).

- The future requirements for improved mapping tables include better identification of the AIM $\rightarrow$ ARM mapping.

- Given such an extended - and computer processable mapping table, it may be the case that the conformance test purposes are generated automatically in the development of the abstract test suite, and may not be required as part of the AP documentation. 


\section{Usage test purposes}

- In addition to conformance test purposes, additionai tests whlch exerclse speclfic, and possibly compiex, combinations of data in the AP may be identified.

- These test purposes are llkely to result from AP (and specificaliy ARM) validation exercises.

- Such test purposes are likeiy to be used in assessing specific CAx systems as potentiai candldates for implementation of the AP.

- Such test purposes are Included in clause 6 of Part 203 as "complex test purposes".

- Recommendation: usage test purposes should be an optional element of Annex G of the AP ("AP Usage Guide"). 
To : Participants in the AP Valldation Workshop Aprll $13-141982$, ISO/IPO meeting in Seattle. April 1992

From : Jon Aas, FEGSLI

Telephone: $\quad+44223237911$

Fax: $\quad+44223234192$

Email:

\section{Position paper: "AP development procedures and formal milestones, will wo over got to the end?"}

\section{Introduction}

The methodology defining the application protocol development process has been progressing very rapidly over the last year. The current version of the AP Guidelines, ISO TC184/SC4MGA N32 (P5), dated 8 January 1992, sets out the structure of the work, defining a number of activities and milestones to be reached in the process of developing an AP within ISO.

For anyone who wants to propose an AP project within ISO, It is necessary to estimate the man time needed to reach the different mllestones. There is no indications as to when the different stages in the AP development should be completed, messured in terms of isO meetings or hours of Vaiidation. Qualificatlon, Editing, etc, Committes offort. In other words, the amount of resources needed to complete an AP, ie bring it up to a DIS status is unknown or open-ended.

This position peper will describe the problem and suggest a possible strategy for the completion of AP projects, based on the AP Guidelines document and the information I have avallable on the AP Qualification Manual (I am walting for an update).

\section{The Goal}

The target is to pln down a polley within WG4PI \& P5 that will allow AP project leaders to estimate the time needed to complete the work and to monitor the progress. Thls will also allow the Qualification and Valldation project to structure its work so it is possible to handle the avalanche of APs that is expected as soon as STEP Version 1.0 is out.

\section{The current structure of the Process for Developing and Quallfying STEP Application Protocol}

The current structure is well defined and serves some well defined purposes:

- It is incremental to ensure that commonally between different APs are identlfied at an early stago

- It Is incremental to allow a comprehensive review procedure at each stage in the AP development process

The incremental nature of the process will provide APs of high quality.

The components of an AP are defined. The development of an AP consists of the development of a succession of components, where each step in the development process bullds upon the precision and documentation of the previous steps.

Each component of an AP proceeds through three baslc steps:

1) define the requirements and evaluation criteria for the component

2) develop the component

3) exercise the criteria to evaluate the component

The AP Development and Approval process is defined in three strends:

- the AP Devolopment Process

- the AP Validation

- the AP Review and Qualification Process 
They are Interlinked, as the AP Revlew and Qualiflcation Process will start when the AP Deveiopment is still in progress. The AP Revlew and Quallfication Process has two phases, A and B.

Phase A: AP Development Revlews, for roviews while the AP components are being developed.

Phase B: WG4/OP AP Qualification when the AP is complote

AP Roview and Quallication Process

\section{Contrlbutors to AP Development Projects}

There are many contributors to the process of developing an AP. This is the list of all contributors and their responsibilities:

Industry Representatives documents requlrements for product data communication and potential AP8, produce a Candidate AP Summary

STEP Experts

Idently correspondence between Industrial requirements and scope and architecture of STEP.

The AP Project Team

develops the AP itself and documents it

SCA PMAG

approves an AP development project. They also monitor the progress of AP projects and provides oversight coordination and resource allocation.

WG4 AP integration Project revlews and ovaluates the ARM. UoF and information requirements WG4 AIM Development Project shall provide technical advice and reviews for AP projects.

WG4 STEP Part Qualitication Project shall provide technical advice and reviews for AP projects.

WG4 STEP Pant Qualification Project Leader is responslble for running the WG4 actlvities to AP completion

WG6 reviews and approves conformance requirements, test group structure and test purposes

\section{Allocation of resources}

The AP Project Leader is the one to define the project duration and allocate resources to different activities.

The AP project, through Its Project Leader, is responslble for requesting resources from WG4 AIM Dovelopment Project and WG4 STEP Part Quallfleation Project for meetings during the development period.

SC4 PMAG will approve the work plan and subsequently commit the necessary resources to support the AP Development and Approval Process.

\section{Mllestones and Scheduled Meotings}

There is a vast number of meetings that are scheduled from the stant to the end of an AP project. Here is a llst of meetlngs given in the chronologlcal onder. indicating who should be present. 


\subsection{The AP Development Process}

A number of meetings are required for the industry speclallsts to document requirements for product data communication and potential APs. No estimates in time and offort is made.

The AP Prolect Team should conduct a set of Industry reviews and evaluations of the AP scope and requlrements. No estimates in time and effort is made.

A lot of home work has to be done by the AP Project Team. This will depend on the area addressed by the AP and the time available for In depth analysis. No estimates in time and offort is made.

A number of meetings are scheduled together with the WQ4STEP Pant Quallfication Project and other contributing to the process.

\subsection{The AP Valldation Procese}

A number of meetings are listed.

\subsection{The AP Review and Quallitication Procese}

Phase A: AP Development Reviews

Actlvity A1: WG4/STEP Part Quallfication Project \& AP Project Team

Activity A2: WG4/AP Integratlon Project

Actlvity A3: WG4/AIM Development Project

Activity A4: WG4/STEP Part Quallfication Prolect

Activity A5: WG4/AIM Development Project

Activity A6: WG4/STEP Part Quallfication Project

Activily A7: WG6

Actlvity A8: AP Project Team, WG convener

Bhase B: WGA/STEP Part AP OUAlification

Actlvily B1: WG4/STEP Part Quallfication Project Loader

Actlvity B2: AP Project Team

Activity B3: WG4/STEP Part Qualification Project Leader

Activity B4: WG4/AP Quallfication Panel \& AP Project Team member(s)

Activity B5: WG4/STEP Part Qualification Project Leader on his own

Activity B6: WG4/AP Qualification Panel members off-site

Actlvity 87: WG4/AP Qualification Panel \& AP Project Te8m

ACtivity B8: WG4/STEP Part Qualification Project Leader on his own 


\section{The document exchange for an AP in ISO}

The entire AP Development and Approval Process is based on a continuous exchange of documents. The documents are produced by several of the contributors in thls game, most notably the AP development team represented by the AP project leader. The structure of the process is here defined in terms of document exchange.

\subsection{The AP Dovelopmont Procese}

Actlvity 1: The result is a document describing the Industrial requirements for an AP or a suite of APs.

Actlvity 2: The results Is an Initial scope definition of an AP or a sulte of APs.

Actlvity 3: A candidate AP Summery submitted to SC4 PMAG. Input to SC4 PMAG from WG2, WG3, WG4

Actlvity 4: Scope and Requlrements Evaluation Report, to 00 into the AP Validation Report.

Activity 5: AP Deveiopment Plan, Including the AP Valldation Plan.

Activity 6: The definitlon of the ARM and UoFs

Actlvity 7: Report on: Information Requirements, ARM \& UOFs submitted to: WG4 AIM Development Project

Actlvity 8: Report on: Information Requlrements, ARM \& UoFs submilted to: WG4 AP Integration Project

Activity 9: Report on: ARM Validation Report, a part of the AP Valldatlon Report, AP Usage Tests

Activity 10: Report on: Group 1 components 8ubmitted to: WG4 STEP Part Quallication Project

Activity 19: Report on: AIC Library and Integrated Resources mapping to the AIM.

Activlty 12: Report on: ARM to AIM Mapping, a part of the AP Validation Report

Actlvity 13: Report on: AlM valldatlon Report, a part of the AP Validation Report

Actlvity 14: Report on: Group 2 components submitted to: WG4 STEP Part Quallfication Project

Actlvity 15: Report on: AP Conformance Requirements, Test Group Structure and Test Purposes. a part of the AP Valldation Repon

Actlvity 16: Report on: Completed AP document submitted, AP Issues Log and AP Vaildation Report

\subsection{The AP Valldation Process}

The AP Project Team should produce an AP Validation Plan. The AP Validation Plan shall be revlew with the WG4 AP Guidelines Project and WG6. The AP Valldation Plan and the resulting AP Validation Report shall be submitted with the complete Draft AP for revlew and acceptance by the WG4 Qualiflcadion Project.

The components are described In the previous sectlon. 


\subsection{The AP Revlow and Quallication Process}

The documents to be produced in the AP Review and Quallfication Process are as foilows:

Phase A: $\quad$ AP Dovolopment Reviews

Activity AB: The Draft AP is is submitted for approval by WG4STEP Part Qualification Project.

Phase B: WG4/STEP Part Qualification Project AP Qualification

Activity B2: A possible undated AP to be distributed to WG4/STEP Part Quallfication Panel members

Actlitty B3.1: WG4/STEP Part Qualification Project Leader develops workshop plan and schedule.

Activity 85: WG4/STEP Part Qualiflcation Project Leader prepares a preliminary qualification report

Activity BB: WG4/STEP Part Quallfication Project Leader completes the AP quallification report and submits the report to the AP Project Team Leader. Editing Committeo and SC4 PMAG.

\section{Discussion}

The current structure of the AP Guidelines document is such that the effort required to achieve an approved AP is scattered around in the document. No effort has been made to quantify the resources needed to complete the job, or how it should be possible to get the various groups to contribute their part.

It is necessary to get the resource requlrements defined and a possible work schedule identified. For anyone doing project management of an AP project, it is important to have this information readily available for the pianning and costing of a project.

The AP Project Leader must be able to forecast the completion of the project, and base his estimates on the avaliability of necessary resources. The AP Project Plan is among the flrst documents an AP Project Leader has to produce for SC4 PMAG approval.

The scheduling should be based on the 150 and ISONPO meeting schedule, knowing that the project will get the necessary resources made avaliable to them at meetings or in the Interim perlod to progress work according to a predefined work plan.

With such a structure of the indlvidual AP projects, It will be possible to predict the need for meetings for the AP Qualification Project Leader and to allocate the necessary resources to the activities. This is absolutely necessary to be able to cope with the expected avalanche of APs following the release of STEP Version 1.0. 


\section{Recommendatlons}

It is Important to get a structure in the AP Development and Approval Process. The following recommendations are made:

Recommendation 1: it is necessary to go through the AP Development and Qualification Process to rationallse the milestones and the work effort. It must be a target for the WG4 Guidelines Project to define a work schedule that will enable an AP Project to go through the process from start to finish within 5-6 150 meotings. ie less that 18 months.

Recommendation 2: A recommended work plan should be developed, to give AP projects a gulde-line for what is required in time and effort to make a DIS AP. This work plan should contain all milestones for completion of the different activities, tied down to named ISO and ISOMPO meetings.

Recommendation 3: Create a set of formal request forms, which each AP Project Leader is expected to use when requesting response from SC4 PMAG or any other contributor to the process

Recommendatlon 4: Get the required man power made avallable to the WG4/STEP Part Quallication Project. This should no longer be based on voluntary contrlbutions, but be fully paide-consuttangy wotk. foll Time weth.

Recommendation 5: Nothing has been defined for the case of unsuccessful reviews, le some of the activities have to be repeated. The probiem of reducing the delay in iterations has to be addressed.

\section{Open issues}

There are still a number of questions to be answered about the entire process. Here are a few:

- Is it possible to simplity the process of AP Development and Approval?

- What happens with an AP effort that has not followed the process assumed in the AP Guidelines document, but has for instance been developed to completion outside ISO? 
The Use of Application Model Validation in Testing a Proposed Standard

\author{
Mary J. Mitchell and Katherine C. Morris \\ National Institute of Standards and Technology \\ Building 220, Room A127 \\ Gaithersburg, MD 20899 \\ (301) $975-3538$ \\ FAX (301) 258-9749 \\ omail: mitchell@cme.nist.gov
}

\begin{abstract}
An Application Protocol (AP) is a spocification of data sharing requirements for a particular application area. The Standard for the Exchange of Product Model Data (STEP) provides an integrated collection of product definitions which allow for the unambiguous description of application data requirements. Application Protocols are designed to permit practical implementations of STEP. However, validation testing is needed to ensure that the technical solutions provided by an AP will work in a practical sense. This validation focuses on the principal mechanism for specifying the data sharing requirements, an application specific information model. The body of the paper describes the process by which an application model is validated.

Application model development and validation is a complex process that relies extensively on human capabilities for analysis, judgement and synthesis of large amounts of diverse information. This process requires the support from automation to produce a technically complete AP. The model validation process and the STEP development methods place unique requirements on the software that will be needed to support the effective testing of STEP. The National PDES Testbed at the National Institute of Standards and Tectnology has undertaken a software project to support this process. The current direction of this project has been formulated from our initial experiences in exercising this process and with software automation for the model validation testing. In addition, this paper introduces the potential contribution that application model validation and validation tools could make to the conformance testing of AP implementations.
\end{abstract}




\section{The Use of Application Model Valldation in Testing a Proposed Standard}

\section{Introduction}

From an information system development perspective, logical data modeling techniques have taditionally served in two roles. The first role is as a method of describing the information requirements of an application system. The second role is as a mechanism for integrating the requirements from a number of applications into a single logical and consistent schema so that data can be shared by multiple applications. The Standard for the Exchange of Product Model Data (STEP) project is developing an international standard' which uses data modeling as the basis for a multi-national and multi-enterprise integration effort. STEP is designed to provide a complete, unambiguous, computer-readable definition of the characteristics of a product throughout its life cycle. STEP product definition specifications are implementation independent, though implementation interface techniques provide the communication mechanisms for applications using file exchanges ${ }^{2}$ or shared databases. Bocause of the diversity of applications that are within the scope of STEP, the integration generally causes extensive changes but the changes are justified if all of the information requirements are supported.

This paper describes a method for ensuring that all application information requirements are met. While much of the terminology is specific to the development methods used for STEP [Danner92, Palmer91], any large scale, multi-enterprise integration activity should find this material useful. The method for validating a logical data model should be applicable even if the data modeling technique is other than one of those used by STEP.

Confidence in a standard by its user community is absolutely essential for a standard to gain acceptance. Validating that user needs are supported by the proposed standard is the foundation for any useful standard. Similarly, confidence that an integrated data model supports user needs is essential in any implementation of any large, complex database system. Significant investments will be required to implement STEP applications. Because of the integration, STEP will contain a number of untried solutions to technical problems. The STEP user community must not ask vendors to develop implementations based on specifications that have unknown levels of risk. Thorough, appropriate model validation testing before standardization can minimize this risk. In addition, this testing will reduce the need to continually 'patch' the standard to correct design flaws uncovered by implementing the standard. The use of proven integrated data models provide the mechanism for controlling these risks. Developers of large integrated databases should want the same sort of proof that their integrated logical data model is correct prior to implementing numerous applications.

The International Organization for Standardization (ISO) is developing ISO 10303 - Industrial Automation Systems: Product Data Representation and Exchange in Technical Committee 184 (TC 184) Subcommittee 4 (SC4) on Industrial Data and Global Manufacturing Programming Languages. For an overview of ISO 10303 refer to Pant 1: Oveniew and Fundamental Principles [ISO1].

2 The initial release of STEP supports only a file exchange interface but an interface for exchange through database transactions is under development. 
An Application Protocol (AP) is a specification for a portion of the product data described by STEP [Palmer91]. APs are the parts of STEP that are to be directly implemented by vendors of software products who adopt STEP. The entirety of STEP consists of all STEP APs along with supporting definitions for the elements that are common to many APs, the specification languages, implementation interfaces, and conformance testing requirements. AP specifications are derived from a set of integrated data models, called Integrated Resources', that contain product definitions in a broad context, e.g. ecross the product life cycle and across manufacturing disciplines. In the AP, these general descriptive definitions for products are interpreted to describe specific data requirements for a given application. Within an AP there is no redundancy and there is consistency with the integrated resources. Due to this consistency, the STEP AP specifications permit product information to be unambiguously exchanged of shared between implementations on dissimilar systems.

Procedures are needed to ensure that the technical solutions provided by STEP APs and integrated resources will work in a practical sense. The term application model' is used throughout this paper to refer to the component information models in an AP or any other domain specific information model with similar properties. In 1991, Mitchell [Mitch91] proposed a methodology for validating STEP AP models. The National PDES Testbed is used to test the validity of application models and the software from its Validation Testing System (VTS) supports this methodology. This document introduces the methodology and shows how the VTS applies the proposed methodology to support AP model validation. The methodology and software build on previous experience with testing STEP from an application's perspective. Another type of testing, the conformance testing of vendor AP implementations, should leverage the VTS as well as some of the outputs from application model testing.

A detailed discusion of the VTS can be found in a series of reports from the National PDES Testbed [Mitch90,Mitch91,Morris91a,Morris91b,Morris91c]. Section 2 of this document gives an overview of the validation testing process for APs. Section 3 describes the activities which comprise the validation testing

3 The initial Integrated Resources in STEP Include: Integrated Resource Fundamental Concepts, General Shape Representation Concepts (which includes geometry and topology), Representation Structures, Product Structure Contiguration Management, Presentation, and General Draughting Concepts. For a technical description refer to Part 41: Integrated Generic Resources - Fundamentals of Product Description and Support [ISO41].

4 The information models that are components of an AP are called application interpreted models (AIMs) and application reference models (ARMs). Other domain specific Information models include context-driven Integrated models (CDIMs) which are used to evaluate STEP resource models [CDIM A1] and various AP precursor models existing outside of the standardization process for purposes such as vendor prototyping. The validation methodology is applicable to these other application models if STEP deveiopment methods are used. The first priority of the VTS project is to support the requirements for validation of AIMs and CDIMs.

- The National PDES Testbed is located at the National Institute of Standards and Technology. Funding for the Testbed Project has been provided by the Depantment of Defense's Computer-Aided Acquisition and Logistic Support (CALS) Otfice. The work described in this document is funded by the United States Government and is not subject to copyright. 
methodology [Mitch91]. The last section describes the future directions for the VTS software, based on experiences with an interim software system used at the National PDES Testbed. These experiences provide a basis for the VTS sottware architecture described by Morris [Morris91c]. Finally, the overlap of software requirements in the validation of application models and in the conformance testing of vendor AP. implementations is discussed.

\section{Overvlew of Valldation Testing}

Application protocol development and testing is a complex process. It involves the synthesis, analysis, and manipulation of large amounts of diverse information. Most of the process relies exclusively on human capabilities for analysis, judgment, and interaction; however, part of this process can and should be automated. The strategy for automation is based on an analysis of the information flow of the AP development and testing process and initial experiences with automation for validation testing at the National PDES Testbod. This section describes the validation process in general. For a more detailed presentation, refer to the proposed AP model validation methodology [Mitch91].

Validation testing of AP information models determines whether the AP does what it is intended to do, i.e., meets the functional requirements that lod to its development. The integrated resources of STEP are also shown to be capable of supporting the application area. The proposed approach is to validate AP models by simulating the behavior of relevant applications using industry contributed data. The validation tests are identified by examining the application processes. The types of data required to perform each activity in the application process are specified in detail. Realistic data fom the application domain is associated with each of these tests. Multiple sets of data may be used to ensure that the expected range and variation of industry uses can be supported by the application model. The data needed to perform a specific process or generated by a specific process is then mapped into the structures defined by the application model. This approach to validation essentially simulates the behavior of an application system interacting with the file or database system that provides data storage. Since an AP is used for data sharing, its performance must be validated against the data access requirements for the application.

The development and validation of an application model can be decomposed into the following seven high-level activities. Some activities may be performed by separate gioups of people. The first two activities establish the application context and construct application data models that will be tested. The next three activities focus on the application model testing, three through five, evaluate the correctness of the application models. Activity 6 controls the identification and resolution of issues against the model. These issues must be resolved to assure confidence in the model. Once the application models have been validated, the remaining AP components can be developed. In the seventh activity, the requirements are defined for what in implementation must be tested for conformance to the standard. All resulting outputs which are required components of an AP are listed in italics.

- For a discussion which focuses on AP development and AP project planning, refer to Development Plan: Applicaiton protocols for Mechanical Parts Production [Stark91]. 
The activity of "Scoping the Application Context" identifies a formal technical boundary for the application model by examining the functions of the application. The boundary is defined by analyzing the general . processes the application performs using an activity model. The activity model, which illustates the scope of the application area, is reviewed by experts in the area to ensure that it reflects common business practices. The scope and requirements guide the determination of what neods to be tested. The results of the activity follow:

- an activity model represented in IDEFO which defines the application processes in the AP, and - a statement of scope and overall requirements.

\section{Model Construction}

During the "Model Construction" activity detailed information models are constructed. Interviews with experts in the application area and reviews of comparable automated systems provide detailed information requirements and usage. These requirements are driven into a detailed information model which is called an Application Reference Model (ARM). Appropriate segments of the existing STEP integrated resource models are identified and interpreted to satisfy the application requirements as specified in the ARM [Danner92]. This interpretation process results in an Application Interpreted Model (AIM). The AIM supports the requirements of the ARM but is based on the information structures from the integrated resource models. The ARM is documented in one of the accepted information modeling formats and the AIM is to be provided in two formats, Express and Express-G [Palmer91, ISO11]. The following outputs are produced from this activity:

- the $A R M$, documented in terms familiar to an application domain expert,

- a formal and documented specification of the AIM in Express, and

- a graphical representation of the AIM in Express-G.

The interpretation process produces an application model that is specific to an application area and also consistent with other phases in a product life cycle. Both the ARM and the AlM are validated. The ARM validation ensures that requirements are valid and that the model can support them. The AIM validation ensures that the interpretation of the integrated resources is correct and that the interpreted resources can support the requirements.

\section{Test Definition}

The result of the "Test Definition" activity is a plan for validating the application model. This information is informative and guides the testing process but it is not computer-processible. The test plan describes how typical users and systems within an application area use information to perform the activities described in the application activity model. Results from expert interviews and automated system reviews are synthesized into significant combinations of information that identify non-redundant and realistic test conditions, called test purposes, which are based on the usage requirements. Each test purpose is a data access request that neods to be satisfied using representative data during the validation process. 
Included in this activity is an identification of the types of and sources for data needed to conduct the tests. The test plan provides the organization to manage the complexity of the required tests.

There are three steps involved in the "Test Definition" activity. In the test planning step, what needs to be tested is decided. A test plan with test purposes for data usage is produced along with a product profile which describes unique characteristics of the product information. The product profile is used for gathering representative test data. In the next two steps, "create cross reference map" and "coverage analysis", additional test details are defined and industry contributed product data is gathered and organized. Separate activities are not defined for these two steps because they do not generate new requirements for software tools, but they are critical steps in validating an application model.

The Test Definition activity produces two additional outputs which are used by the next activity: 1) a cross reference map, and 2) a coverage feodback report. The cross reference map indicates the correspondence between the application model and the representative test data. The creation of the cross reference map frequently uncovers major structural flaws in the application model. The coverage analysis of the representative test data reveals unused segments of the application model. If the AP project cannot identify data which corresponds to these segments, then the application model needs to change. Either the model was misunderstood and requires clarifying documentation; or additional searches for corresponding data are necessary. Ultimately, the unused segments are removed from the application model when their information requirements cannot be verified.

This activity produces four outputs:

- an overall test plan with usage test purposes,

- a product profile and the identification of representative test data meeting these criteria,

- a cross reference map to correlate the test data with the application model, and

- a report which describes issues and needed improvements to the application model.

\section{Test Case Data Generation}

During the "Test Case Data Generation" activity, test case data is assembled or built from the contributed product data which has been selected by using the characteristics identified in the product profile. The objective is to identify where in the application model the representative product data will reside and if the information structures provided in the application model can accommodate it. Each piece of industry-contributed data should have a single, logical place in the model. In initial testing experiences [PDES90] much of the data was not available in electronic form so the test case data was prepared by hand. This was the most labor-intensive and error-prone activity of the entire process, but potentially the most reusable for conformance testing. Many deficiencies in the application model are uncovered by associating industry contributed product data with the model's information structures.

The computer-processible output of this activity is the test data. This data directly supports the next activity, "Test Execution and Analysis". The process that makes that data available for test execution should not need turther human intervention. Also in this activity, the test purposes for usage are fully 
detailed and documented as abstract test cases. 'This activity results in the following output:

- detailed test data in a format suitable for processing by the VTS software, e.g. STEP exchange file format [ISO21],

- usage abstract test cases, and

- a report which describes issues and needed improvements to the application model.

\section{Test Execution and Analysis}

The "Test Execution and Analysis" activity involves the development, execution, and analysis of the test cases against the application model. In order to execute the test cases, a computerized testing environment needs to be established and the test cases need to be formally specified with respect to the testing environment. Analysis of the test cases involves comparing the test results to the expected results to determine the validity of the application model. In addition, general statements about what any implementation of the AP must support are documented. This activity produces the following results:

- validation test reports,

- additional usage abstract test cases,

- improved test case data for reproducing test results,

- executable test cases for reproducing test results, and

- a report which describes issues and neoded improvements to the application model.

\section{Vl. Model Reflnement}

The "Model Refinement" activity resolves issues that were uncovered during the testing process.

Alternative solutions are proposed and the best solution is selected. Once there is agreement on how to resolve an issue, the model is modified and a new model is released for validation testing. The process of resolving an issue may replicate many of the preceding steps, e.g. the addition of an entity for resolving an issue might cause additional industry contributed data to be gathered and new test cases to be built. This activity results in the following information:

- the refined application model (ARM or AMM),

- an issue resolution statement describing the selected solution and supporting rationale, and

- refined test purposes, abstract test cases, and executable test cases.

Model validation testing is an iterative process. The end result of the process is an application model suitable for inclusion in a STEP AP. The model must be both useful and usable to be part of the standard. The involvement of a variety of application experts in the validation process helps to ensure that the model is useful. There should also be reviews by application experts who were not members of the

7 The abstract test case and test purpose in conformance testing is a related concept but the intended purpose is different. In validating an application model, the intended purpose is to evaluate how well the model functions for supporting its intended scope. In conformance testing. the intended purpose is to evaluate if an implementation supports all of the required features of a standard. 
AP project. To ensure that the model is usable, validation testing should be repeated until the model satisfactorily supports the information needs identified in the test plan.

The validation of an application model is dependent on the application area under consideration, but the . validation process itself is constant and many aspects of it can be automated or supportod by automation. Due to the nature of the standard being developed, it is mandatory that some parts of the process are automated. The standard will enable the automatic sharing of data. Therefore, the ability to automatically access data using the application model neods to be verified. Section 3 below discusses how this can be accomplished.

\section{VIl. Speclfication of Conformance Requlrements}

The "Specification of Conformance Requirements" activity is performed when there is confidence that the application model provides the functional capabilities that were specified In its scope. Conformance requirements specifiy all characteristics that must be satisfied by a conforming implementation of the AP. The three required components for an AP which fall within "Specification of Conformance Requirements" are:

- a conformance clause which specifies overall requirements for completeness and conformance,

- test purposes for conformance and completeness testing of vendor implementations,

- Protocol Inplementation Conformance Statement (PICS) proforma which is a checklist for identifying optional characteristics that a vendor may claim to implement.

No further detail is provided since there are many unresolved issues relating to this topic (see Annex C of the "Guidelines for Development and Approval of STEP Application Protocols" [Palmer91] for further enumeration).

\section{Automation for the Valldatlon Testing Methodology}

This section describes the automated dataflow within the VTS at the National PDES Testbed. The software, which supports the validation testing process, simulates the information access requirements for the application area being tested. The VTS software will provide a controlled environment for model validation testing, thereby reducing the potential for introducing errors into the process. The VTS software and the control of the supporting environment will also reduce the level of computer sophistication and interaction needed so that the users of the system will be able to concentrate on validating the application models.

The primary requirement of this process is the capability to manipulate and represent an application model and associated data for a variety of purposes. Therefore, many functional requirements [Moris91a] such as the ability to display and access the contents of models written in Express [ISO11] and the ability to manage the versions of documents and other files, are common among various activities. Some of these requirements such as word processing for preparation of documents, database access and persistent storage, and computer-aided design analysis of geometry, are not unique to STEP 
and are available in commercial systems. For these requirements, commercial systems will be used and integrated with the VTS software. Other requirements that are unique to STEP are either available from related projects or will be developed for the VTS.

Each activity of the validation testing process consumes and produces specifications or data. A subset of this material is directly processible and can be used to automate the activities. This automation parallels the flow of information between the activities described in Section 2. Table 1 lliustrates Information Inputs and outputs; the entries in bold represent the computer-processible portion of the Information flow between the activities. The remainder of this section focuses on only those portions which are currently computer-processible. For a more general discussion of the information flow for model validation, see Mitchell [Mitch91]. 


\begin{tabular}{|c|c|c|}
\hline & INPUT & OUTPUT \\
\hline $\begin{array}{l}\text { Scoping the Application } \\
\text { Context }\end{array}$ & Application Requirements & $\begin{array}{l}\text { Scope \& Requirements Statement } \\
\text { Applicattion Activty Model }\end{array}$ \\
\hline Model Construction & $\begin{array}{l}\text { Scope \& Requirements Staternent } \\
\text { Applleation Activty Modil } \\
\text { integrated Resource Hodvls }\end{array}$ & $\begin{array}{l}\text { Appilcation Modils (Le. ARU, AlM } \\
\text { Graphic Intormation Modil }\end{array}$ \\
\hline Test Dofinition & $\begin{array}{l}\text { Application Requirements } \\
\text { Scope \& Requirements Statement } \\
\text { Application Activty Model } \\
\text { Application Model in Express }\end{array}$ & $\begin{array}{l}\text { Test Plan with Test Purposes } \\
\text { Product Profile } \\
\text { Contributed Product Data } \\
\text { Cross Roference Map } \\
\text { Model lssues }\end{array}$ \\
\hline Test Case Data Generation & $\begin{array}{l}\text { Scope \& Requirements Statement } \\
\text { Application Activity Model } \\
\text { Application Model In Expreas } \\
\text { Product Profile } \\
\text { Test Plan with Test Purposes } \\
\text { Contributod Product Data (1.e. LES filea) } \\
\text { Cross-Reterenco Map }\end{array}$ & $\begin{array}{l}\text { Tost Cane Data (Lo. STEP fines) } \\
\text { Abstract Test Cesses } \\
\text { Model lssues }\end{array}$ \\
\hline $\begin{array}{l}\text { Test Exocution and } \\
\text { Analysis }\end{array}$ & $\begin{array}{l}\text { Scope \& Requirements Statement } \\
\text { Appllcsiton Activty Model } \\
\text { Appllcatlon Model In Express } \\
\text { Test Plan with Test Purposes } \\
\text { Abstract Test Cases } \\
\text { Test Case Data (l.e. STEP files) }\end{array}$ & $\begin{array}{l}\text { Executable Teat Cases } \\
\text { Validation Report } \\
\text { Modal issues } \\
\text { Refined Abstract Test Cases \& Teot Case } \\
\text { Data } \\
\text { Tool Enhancoment Requirements }\end{array}$ \\
\hline Model Refinement & $\begin{array}{l}\text { Model Issues } \\
\text { Appllcation Model in Express }\end{array}$ & $\begin{array}{l}\text { Appilication Model In Express } \\
\text { Model Log with Resolutions }\end{array}$ \\
\hline Conformance Requirements & $\begin{array}{l}\text { Appilication Model In Express } \\
\text { Abstract Test Cases } \\
\text { Tost Case Data }\end{array}$ & $\begin{array}{l}\text { Conformance Clause } \\
\text { Abstract Test Suite } \\
\text { Model lssues }\end{array}$ \\
\hline
\end{tabular}

Table 1 information Flow Botween noder Developinent and Validation Acivities

Figure 1 below, illustrates the relationship between the activities and the currently computer-processible information flow.

The Model Construction activity produces application models in both human and computer-interpretable formats. Currently only the Express version of the application model is directly used as a basis for the software. The STEP integrated resource models are also represented in Express and supply a basis for the application model being developed and validated. If additional computer-processible representations for these outputs were available, such as a test notation for abstract test cases, the amount of automation could be increased. 
The Test Definition activity involves a great deal of human interaction, synthesis, and analysis. It is the least automatable activity in the process. The automation is limited to assistance in referencing the application and activity models and in the preparation of documentation. The work being done on conformance testing by the developers of STEP includes a formal test notation language that may increase the ability to provide additional automated assistance.

The primary automation for the Test Case Data Generation activity is for assistance in preparing test data. The industry contributed data is represented in many formats, but to be usable by validation testing, the test case data must be formed into a STEP exchange file format [ISO21] or loaded into a database system which has been built to manange STEP data structures. A reliable and efficient way to receive a limited portion of this data, principally geometric entities, is in the form of an Initial Graphics Exchange Specification (IGES) [IPO91] file extracted from a CAD system. IGES files can be translated to STEP exchange files [Breese91]. Additional contributed data needs to be prepared manually to complete the information required for the application model.

In the Test Execution and Analysis activity, executable test cases are generated, executed, and the results analyzed. This activity allows for a high degree of automation. The typical testing scenario follows:

1. the application model and test data are represented in a database;

2. executable tests are specified in the database system's query language;

3. the queries which simulate typical application data access requirements are executed; and

4. the results are analyzed, issues against the application model are documented, and a validation report is generated.

The Model Refinement activity leads to a new application model and may contribute to refinements to the 
STEP integrated resource models. Throughout the validation testing process, any deficiencies in the application model, the test cases, or the test environment are documented, and appropriate enhancements are made. Appropriate steps in the validation process are repeated using the refined application model and test case data for any test purpose that was affected by these refinements.

The Conformance Requirements activity is still evolving. The current understanding of what should be accomplished in this activity is described in Palmer [Palmer91]. There is potential for rousing test case data, abstract test cases and VTS tools for these purposes. The intended purpose of this activity is to specify all of the requirements that a vendor implementation of an AP must satisfy. The VTS project efforts will evaluate these requirements when they become available.

\section{Model Validatlon Testing at the Natlonal PDES Testbed}

The National PDES Testbed has been used for the validation testing of STEP application models since 1989. The sottware currently in place in the National PDES Testbed [Breese91] provides some of the automation desired. An important point to make is that all of the software is being developed to generate a test enviroment for the schema under test. While the current software will support any data model that has been developed in Express, knowledge of the other data modeling techniques used within STEP has contributed to the design. This section summarizes the direction for future improvements and additions to the validation testing soltware at the Testbed. This direction is based on past experiences with the software and STEP development methods, which helped clarify the needs for the validation testing sottware.

\subsection{Experiences with the Interim System}

The interim software employed in the validation testing of application models consists of a set of independent tools which operate in a variety of computer environments. The current method of sharing data in the testing process is by exchanging data files between these tools. This requires data translation and manual intervention, which introduces the potential for errors and inconsistencies, every time data is processed in the testing activities. Moreover, the process of importing and exporting between tools is time-consuming.

The automation for this testing process is currently provided by software tools which translate the application model and the test data among a number of formats [Clark90a,PDES91]. The sottware includes:

- an Express compiler and translators for representing the application model;

- an editor for STEP data [Clark90b] which structures the information as specified in Express for the application model;

- a relational database [Date90] which provides data access and storage management along with a query capability;

- a STEP exchange file parser and loaders for populating the STEP oditor and database;

- Export facilities for extracting data from the editor or database into STEP exchange files;

- an IGES to STEP translator for converting digital product data from a Computer-Aided Design system to STEP; and 
- a visualizer of geometric model data for display of a very limited set of STEP data.

The interim system has some of the needed functionality but provides unacceptable performance for some of the functions. The most significant improvements to the current system can be made by replacing the STEP editor and database management system with improved and integrated components. Both tools have suffered from significant performance problems with the large sets of complex data typical of engineering uses [PDES90].

In addition the STEP editor and database system currently use different data representation paradigms to represent the application model and its associated data. This places a burden on the users to understand the different representational formats and the relationship between these formats.

\subsection{Future Dlrections for the Model Valldation Software}

The software neods for the STEP AP model validation process can bo divided into two categories:

1. automation of the validation process by simulating the data access requirements of the application; and

2. automation to support the validation process, through assistance for preparing documentation and for referencing and browsing the application or integrated resource models.

The first category is a mandatory requirement for effective validation testing. The tests resulting from the process must be computer-processible and repeatable. These tests refiect the intended usage of the application model. Software for this purpose is the first priority for future implementation efforts.

The second category is partially met in the current system by word processing and drawing packages. These solutions provide limited support for these functions and leading to inefficient use of human resources. However, these automation needs will not be addressed until the first category is supported.

Two tools are most important for supporting the first category of automation, the simulation of data access based upon application information requirements:

1. a STEP data editor, and

2. a database system with a query capability.

Current efforts for the VTS software focus on developing an improved and integrated STEP data editor. This editor is being developed so that it will integrate with a database system. However, the editor will not depend on having a database system. The VTS software will provide an integrated set of functions which will provide a more effective and efficient environment and one more capable of simulating the date access needs of the application area. In summary the VTS software will make improvements over the interim sottware in the following areas:

- performance, in terms of both computation time and reliability;

- workilow automation to eliminate manual intervention where possible;

- more sophisticated support for editing of STEP data to reduce inconsistences in the data; 
- error checking to reduce the potential for errors and improve error detection;

- expansion of functionality to address the needs of model scoping, model construction, and model refinement;

- provision of a single interface to the software, which will reduce the effort needed to learn the . tools; and.

- adoption of a data representation paradigm that resembles Express more closely than the relational paradigm.

When completed and tested, the VTS software and supporting documentation will be made available through the National PDES Testbed STEP On-line Information System [Katza1], as are the NIST developed portions of the interim system.

\subsection{Future Directions for AP Validation Methods}

There are two areas where additional effort in defining validation methods are needed. These are:

1. validation techniques for the AP Conformance Testing requirements; and

2. definition of the relationship between AP model validation testing and the specification of AP conformance tests.

\section{Summary}

The validation testing of data models is needed to ensure that the technical solutions provided by integrated model will work in a practical sense. An Application Protocol (AP) within STEP is a specification of data sharing requirements for a particular application area. Application Protocols are designed to permit practical implementations of STEP. The model validation focuses on the principal mechanism for specifying the data sharing requirements, an application specific data model. The body of the paper describes the process by which an application model is validated.

Application model development and validation is a complex process that relies extensively on human capabilities for analysis, judgement and synthesis of large amounts of diverse information. This process requires the support from automation to produce a technically complete AP. The model validation process and the STEP development methods place unique requirements on the sottware that will be needed to support the effective testing of STEP. The National PDES Testbod at the National Institute of Standards and Technology has undertaken a software project to support this process. The current direction of this project has been formulated from our initial experiences in exercising this process and with software automation for the model validation testing. In addition, this paper introduces the potential contribution that application model validation and validation tools could make to the conformance testing of AP implementations.

\footnotetext{
- No liability clause
} 


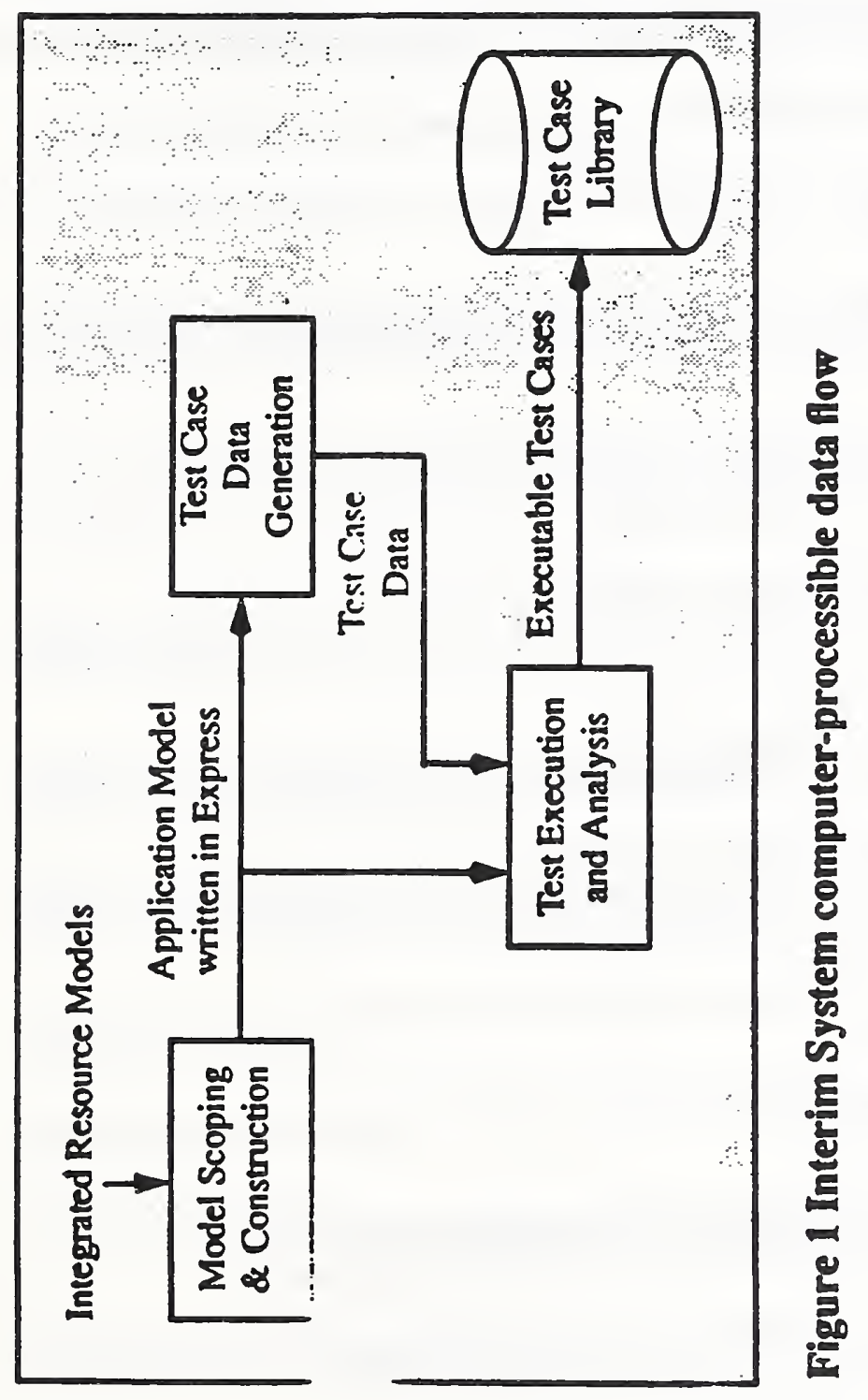


Putorences

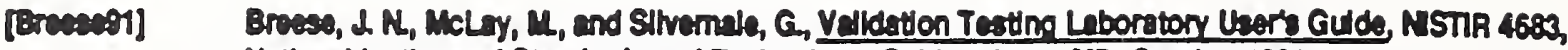
Matbond hat tut of Standards and Technology, Guthoreburg, MD, October 1001.

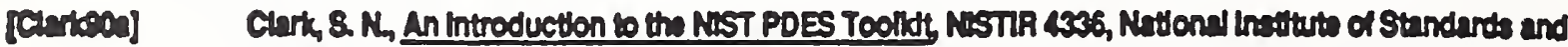
Technology, Gathereburg, MD, Lyy 1900.

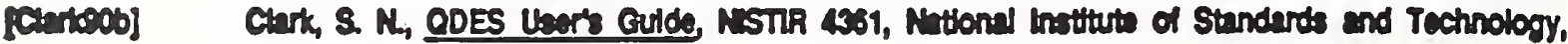
Caltherebuing, MD, dun 1200 .

[Dannarez] Dannw, W. F. and Yang, Y, STEP Dovelopment Methods: Recource Integration and Appilleation Interpretation Working Dret, LO TCI84SCAWG5N31, danuary 1992

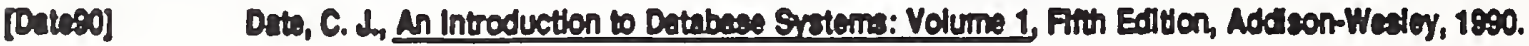

[PO91] The hittal Graphice Exchenoe Spectificatton DGES, Verion 5.1, IGESPDES Organtation, NCGA, Falriax, VA, September 1991.

[ISO1] $\quad$ LSO 10303 Industrlal Automation Syctems - Product Data Ropresentation and Exchanoe - Part 1: Overviow and Fundamental Princlples, Working Draft, Vereion 2, 150 TC184SCAWGPLAGN50, Mason, $H_{1}$ cd., Decembor $28,1991$.

[S011] \$SO 10303 Industrlal Automatton Systems - Product Data Representation and Exchanoe - Part 11: Description Methods: The EXPRESS Lanquage Reforence Manual, Committeo Drath ISO TC184SCANG5N14, Splby, Pq od, Aprll 29, 1981.

[ISO21] LSO 103003 Industrial Automatton Syetems - Product Date Representation and Exchanoe - Part 21: Clear Text Encoding of the Exchanoe Structure, Comrittee Drat ISO TC184SCA, Van Maanen, Jar, ed., March 12, 1991.

[ISO31] - ISO 10303 Industrial Automstion Systems - Part 31: Conformance Teating Methodotogy and Framework: General Concepts. Wortung Drath, LSO TC184SCANGGNES, Owon Jn ed., January 2, 1992

[ISO41] LSO 10303 Industrial Automation Syatems - Product Dita Representation and Exchange - Pan 41: integrated Generic Resources: Fundamentals of Product Deserlption and Support Comnittee Drath,

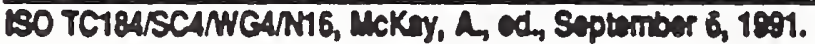

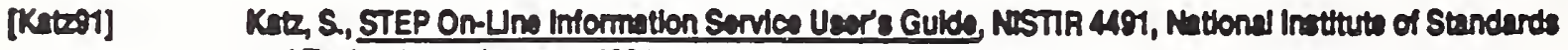
and Technology, danuary, 1991.

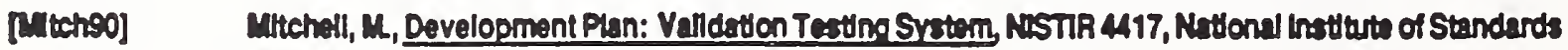
and Technology, September 1990.

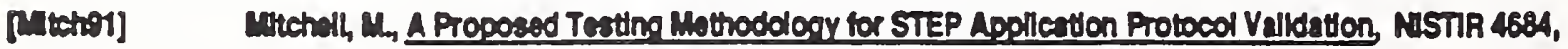

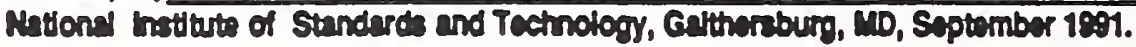

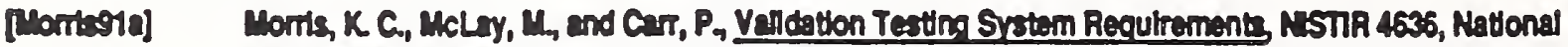
inattute of Standards and Technology, Gelthereburg, MD, Septenber 1991.

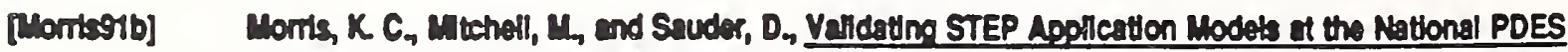
Testbed, MSTIR 4735, Mational thattuth of Standards and Technology, Galthersburg, MD, December 1991. 


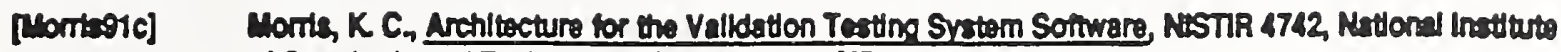
of Standerde and Technology, Gelthereburg, MD, Decomber 1909.

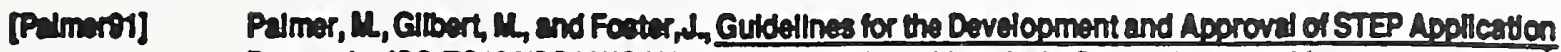

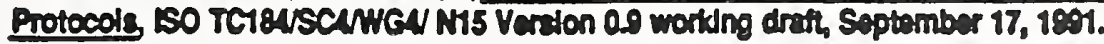

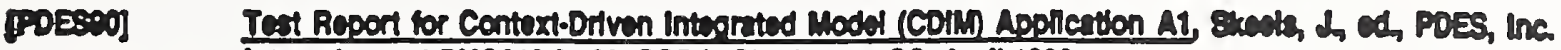
internd report PICO1201.00, SCRA, Charteston, SC, April 1\%\%.

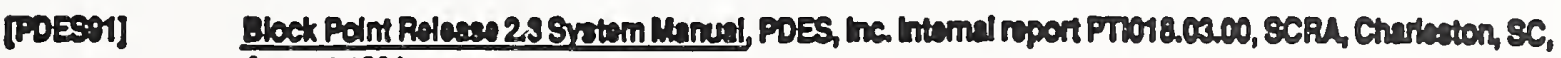
Aevour 1991.

[Starto91] Stark, $C$, and Mitchell, $M$, Development Pien: Apollcation Protocol for Mechanical Parts Production MSTIR 4628, National Inattusto of Standards and Technology, Gaithenburg, MD, Juty 1991. 

